\title{
Perinatal essential fatty acids and atopy
}

Citation for published version (APA):

van Gool, C. J. A. W. (2002). Perinatal essential fatty acids and atopy. [Doctoral Thesis, Maastricht University]. Universitaire Pers Maastricht. https://doi.org/10.26481/dis.20021030cg

Document status and date:

Published: 01/01/2002

DOI:

$10.26481 /$ dis.20021030cg

Document Version:

Publisher's PDF, also known as Version of record

\section{Please check the document version of this publication:}

- A submitted manuscript is the version of the article upon submission and before peer-review. There can be important differences between the submitted version and the official published version of record.

People interested in the research are advised to contact the author for the final version of the publication, or visit the DOI to the publisher's website.

- The final author version and the galley proof are versions of the publication after peer review.

- The final published version features the final layout of the paper including the volume, issue and page numbers.

Link to publication

\footnotetext{
General rights rights.

- You may freely distribute the URL identifying the publication in the public portal. please follow below link for the End User Agreement:

www.umlib.nl/taverne-license

Take down policy

If you believe that this document breaches copyright please contact us at:

repository@maastrichtuniversity.nl

providing details and we will investigate your claim.
}

Copyright and moral rights for the publications made accessible in the public portal are retained by the authors and/or other copyright owners and it is a condition of accessing publications that users recognise and abide by the legal requirements associated with these

- Users may download and print one copy of any publication from the public portal for the purpose of private study or research.

- You may not further distribute the material or use it for any profit-making activity or commercial gain

If the publication is distributed under the terms of Article $25 \mathrm{fa}$ of the Dutch Copyright Act, indicated by the "Taverne" license above, 
Perinatal essential fatty acids and atopy 
ISBN: 90-9016159-7

Lay-out: Cobie Martens, UM Epidemiologie, Maastricht Cover design: Madonna con Bambino, Michel Angelo Printing: Datawyse | Universitaire Pers Maastricht

O 2002, C.J.A.W. van Gool

All rights reserved. No part of this thesis may be reproduced or transmitted in any form or by any means, electronic or mechanical, including photocopying, recording or any information appropriate, of the publishers of the publications. 


\title{
Perinatal Essential Fatty Acids and Atopy
}

\author{
ter verkrijging van de graad van doctor \\ aan de Universiteit Maastricht, \\ op gezag van de Rector Magnificus, \\ Prof. dr. A.C. Nieuwenhuijzen Kruseman, \\ volgens het besluit van het College van Decanen, \\ in het openbaar te verdedigen op \\ woensdag 30 oktober 2002 om 14.00 uur
}

door

Christella J.A.W. van Gool 


\section{Promotor}

Prof. dr. ir. P.A. van den Brandt

\section{Co-promotor}

Dr. C.T.M.C.N. Thijs

\section{Beoordelingscommissie}

Prof. dr. G. Hornstra (voorzitter)

Prof. dr. C.E. Blanco

Prof. dr. J.W Cohen Tervaert

Dr. A.D.M. Kester

Dr. A.P. Oranje (Sophia Kinderziekenhuis Rotterdam)

\section{nivtim}

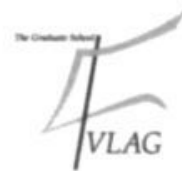

The studies presented in this thesis were performed at the Nutrition and Toxicology Research Institute Maastricht (NUTRIM) which participates in the graduate school VLAG (Food Technology, Agrobiotechnology, Nutrition and Health Sciences) accredited by the Royal Netherlands Academy of Arts and Sciences.

The printing of this thesis was kindly supported by financial contributions of Friesland Nutrition Research and Stichting Nationaal Huidfonds. 


\section{CONTENTS}

1 Introduction

2. Perinatal essential fatty acid status and the development of atopy at age one year in breastfed infants

3. Gamma-linolenic acid supplementation to prevent atopy - a randomized controlled trial in infants at high familial risk

4. Determinants of neonatal IgE level - parity, maternal age, birth season and perinatal essential fatty acid status

5. Oral essential fattv acid sunblementation in atonic dermatitis - a meta-analvsis of placebo controlled trials

6. General Discussion

Summary

Samenvatting

Dankwoord

Curriculum Vitae

About the author 
Introduction 
Chapter 1 


\section{BACKGROUND}

Atopic diseases (atopic dermatitis/ milk scab, food allergy, hayfever and other ainway allergies, and allergic asthma) have been increasing worldwide in the past decades, most predominantly in the Westem world and particularly amongst children.' It is generally believed that the causes for this increase are found among factors in the environment. Plausible causative factors should be able to polarize the immune system toward an allergic sensitization. Furthermore, these causative factors should be distributed in nature in such a way as to explain the reported differences in the prevalence of atopic diseases amongst different populations. ${ }^{2}$ As a result some hypotheses have been formulated and the hygiene hypothesis seems to be the most probable cause of the increase in atopic diseases. However, there are also other factors such as altered gut flora and dietary components that may play a causative role. Of the latter are of particular interest changed dietary habits concerning fatty acids that may play a role in immune responses. ${ }^{2}$

This thesis focuses on the role of essential fatty acids on early development of atopy. The aim of this introduction is to provide a short overview of atopy, the possible role of essential fatty acids herein and also other hypotheses for the increase of the prevalence of atopic diseases are briefly discussed.

\section{ATOPY}

The definition of "atopy" was presented in a publication in the early 1920s by Coca and Cooke (3). In this paper their definition was stated as follows:

"Dr Cooke suggests a subdivision of forms of hypersensitiveness into a 'normal' group and an 'abnormal' group. The group of abnormal hypersensitiveness includes anaphylaxis, the hypersensitiveness of infection and those idiosyncrasies that are controlled by the dominant gene as demonstrated by Cooke and Vandeveer. This latter subgroup evidently needs a special term by which it may be conveniently designated. The Greek word aromia, from which the term was derived, was used in the word of a strange disease."

As result of the development of the field of allergy it became clear that this definition needed modification. In 2001 a revised nomenclature for allergy was suggested by the European Academy of Allergology and Clinical Immunology (EAACI) in a position statement. The general accepted definition used in the past decades was described as follows ${ }^{4}$ :

"Atopy is a personal or familial tendency to produce IgE antibodies in response to low doses of antigens, usually proteins, and to develop typical symptoms such as asthma, minoconjunctivitis, or eczema/dermatitis."

However, in this position statement it was acknowledged that atopic dermatitis is not always associated with increased IgE levels. It was stated that "in current dermatology, 'atopy' is a clinical diagnosis, and a patient can be called 'atopic' without knowledge of skin prick tests or the presence of IgE antibodies in his serum." ${ }^{4}$ We will use the term atopy in this sense, and make a distinction between atopic disease (defined by its clinical symptoms) and serum IgE levels as one of the associated pathobiological traits.

Some hypotheses concerning the increasing prevalence of atopic disease relate to changes in the diet. For over fifty years already, in particular essential fatty acids have been the focus. 
Before a brief overview of essential fatty acids will be given, we will elaborate on the main outcome of this thesis, atopic dermatitis.

\section{ATOPIC DERMATITIS}

The diagnosis of atopic dermatitis (AD) by a physician depends on the history and physical examination, together with a familial history of atopy. Immunological tests, such as determination of total serum $\mathrm{IgE}$ and immediate skin test reactivity, have limited usefulness. Elevated total serum $\mathrm{IgE}$ can occur in a variety of skin diseases and in the hyperimmunoglobulin $\mathrm{E}$ syndrome ${ }^{5}$ and normal total serum $\lg \mathrm{E}$ can occur in up to $20 \%$ of the patients with $A D .^{6}$ Furthermore, total serum $\mathrm{IgE}$ is raised in people who have been infected with parasitic infections. ${ }^{7}$ In population studies asymptomatic people are found with high IgE in a certain percentage. ${ }^{6}$ Immediate skin prick tests are sometimes misleading because the antigen battery in skin prick tests is variable and the interpretation of results controversial. Negative skin prick test responses however, can be useful in excluding a diagnosis of $A D{ }^{8}$ In the first years of life $A D$ is the most common atopic disease. Of the children who will eventually develop AD, $60 \%$ have symptoms in the first year of life and $85 \%$ in the first five years. Approximately $20 \%$ of them have a food hypersensitivity compared to $8 \%$ of the children in the normal population. ${ }^{9}$

In infancy $A D$ is usually manifested between 3 and 6 months of age by a red, sometimes scaly, rash on the cheeks. The wrists, extensor aspects of the legs, arms and neck are also frequently involved. The general aspect of the rest of the skin is that it is often dry and itchy. Scratching makes $A D$ worse and in severe cases weeping lesions, secondary bacterial infection and crusting may be present. Chronic inflammation and scratching also results in lichenification (epidermal thickening and increased skin markings). The skin features of $A D$ often follow a relapsing course with remissions and exacerbations. When specific food hypersensitivity is found (most commonly for cow's milk) elimination of the food allergen from the diet may lead to partial or total remission of $A D$. Older children and adults characteristically have a red, scaly eruption with a distribution of the flexural limb surfaces, particularly affecting the antecubital and popliteal fossae. Sometimes the eyelids, neck, forehead and chest are also involved. ${ }^{8}$

Several physicians have tried to define diagnostic criteria for AD. According to Hanifin and Rajka ${ }^{10}$ a patient with $A D$ have to meet at least three of the following four aspects ('major criteria'); 1) pruritus, 2) typical morphology and distribution (in infants facial and extensor), 3) chronic or relapsing dermatitis, 4) personal or family history of AD; together with three of a list of 23 'minor criteria', amongst others; xerosis, immediate (type I) skin reactivity, elevated total serum IgE and food intolerance. Williams et al. ${ }^{11}$ reported on the UK Working Party's Diagnostic Criteria for Atopic Dermatitis, having systematically assessed the validity of the Hanifin and Rajka criteria. Using multiple regression techniques, they derived a minimum set of diagnostic criteria for AD. Their guidelines are as follows; in order to be diagnosed with AD the patients should at least have: an itchy skin disorder, plus three of the five following aspects: 1) history of the involvement of skin creases such as folds of elbows, behind the knees, fronts of ankles or around the neck (including cheeks in children under 10), 2) a personal or familial history of asthma or hay fever, 3) a history of general dry skin in the last year, 4) visible flexural dermatitis, 5) onset under the age of 2 . These criteria have a higher 
specificity than the Hanifin and Rajka criteria, $93 \%$ compared to $78 \%$, but a slightly lower sensitivity, $88 \%$ compared to $93 \% .{ }^{11-13}$ Furthermore, these criteria can be put in a regression equation (as described in the methods section of chapter 2 and 3 ), which than provides the probability of AD score for a particular patient.

\section{ESSENTIAL FATTY ACIDS}

Fatty acids consist of a chain of carbon atoms with a methyl head group on one end and a carboxyl tail group on the other end. They differ in length, indicated by the number of C. atoms, and structure. Fatty acids can be saturated or unsaturated, dependent of the absence or presence of double bonds between the carbon atoms. A variety of notations are available to indicate fatty acids. In this thesis the following system is used: ${ }^{14}$ in the notation of linoleic acid (LA), written as 18:2n-6, "18" refers to the number of carbon atoms in the fatty acid. The degree of desaturation is indicated by the number of double bonds " $: 2$ ". The first double bond counted from the methyl end $(n)$ is at the $6^{\text {th }}$ position, hence n-6.

Fatty acids are metabolized by an alternating sequence of desaturation (i.e., removal of two hydrogens) and elongation (i.e., addition of two carbons) (Figure 1). Certain unsaturated fatty acids are essential for human health, because they need to be consumed with the diet as they cannot be synthesized de novo by human. ${ }^{15}$ Two separate, so-called families of essential fatty acids exist: the n-6 fatty acids and the n-3 fatty acids (Figure 1). Both families have a parent fatty acid, linoleic acid (LA, 18:2n-6) and alpha-linolenic acid (ALA, 18:3n-3), which are on top of the chain of desaturation and elongation processes. First of all, LA and ALA are desaturated by delta-6-desaturase (D6D) into gamma-linolenic acid (GLA, 18:3n-6) and stearidonic acid (18:4n-3), respectively. D6D is considered to be the rate-limiting step in the whole chain of desaturation and elongation. ${ }^{16}$ GLA and stearidonic acid are then further elongated and desaturated into longer chain, more unsaturated derivatives, the long chain polyenes (LCPs; with more than 20 carbon atoms and at least one more double binding than the parent fatty acid). N-6 and n-3 fatty acids compete for the same enzymes, where the enzymes have a higher affinity for the n-3 fatty acids. Even though humans can produce n- 6 and n-3 LCPs from their parent fatty acids LA and ALA, uptake from the diet is much more effective. ${ }^{15}$ Thus, it is important that LCPs are also taken up from the diet. Even though some experts in the field restrict the definition of essential fatty acids to LA and ALA alone, others define EFAs as LA, ALA, GLA, 18:4n-3 and their LCPs together. In this thesis we will use the latter definition. EFAs are important structural and functional components of membranes, where they influence membrane fluidity and the activities of membrane bound receptors, enzymes and ion-channels. In addition, arachidonic acid (AA, 20:4n-6), dihomogammalinolenic acid (DGLA, 20:3n-6) and eicosapentaenoic acid (EPA, 20:5n-3) are precursors for biologically active substances, namely prostacyclins, prostaglandins, leukotrienes and thromboxanes (Figure 1).

To assess whether the status of the different essential fatty acids is optimal, different EFA status markers have been developed;

1. Mead acid (20:3n-9). If no sufficient parent EFAs or their LCPs are consumed from the diet oleic acid $(18: 1 n-9)$ is further converted through the same elongation and desaturation pathways as the essential fatty acids, ${ }^{15}$ consequently increasing the levels 
of Mead acid. Therefore, Mead acid (as weight $\%$ of total fatty acids) is a marker for general EFA status.

2. Osbond acid (22:5n-6). Because the production of Osbond acid (22:5n-6) from LA increases in case of $n-3$ shortage, Osbond acid (as weight $\%$ of total fatty acids) is considered to be a marker of a not optimal n-3 status. ${ }^{17,18}$

Besides these general two indexes, two complementary indexes are considered to indicate docosahexaenoic acid (DHA, 22:6n-3) status; ${ }^{19}$

1. DHA sufficiency index. It has been shown that a low status of $22: 6 n-3$ is accompanied by a compensatory increase in $22: 5 n-6$. Therefore, the $22: 6 n-3 / 22: 5 n-6$ ratio is an indication of sufficient supply of DHA intake. ${ }^{17.18}$

2 DHA deficiency index. A shortage of $22: 6 n-3$ is also accompanied by an increase in the conversion of $22: 4 n-6$ into $22: 5 n-6$ because the enzyme system of desaturation and elongation of EFAs will take on the n-6 EFAs in absence of $n-3$ EFAs. ${ }^{19}$ The DHA deficiency index is defined as $22: 5 n-6 / 22: 4 n-6$.

\section{EFA IN INFANT NUTRITION}

Because of the rapid tissue growth during fetal development and in early childhood adequate supply of EFAs is essential. During pregnancy, maternal stores and dietary intake of EFAs of the mother are of importance in ensuring that the fetus has an adequate EFA status at the time of birth. ${ }^{15}$ After birth the infant is dependent on the supply from breast milk or artificial formula. The fatty acid composition differs considerably between these breast milk and formula milk. Furthermore, breast milk composition, including fatty acid composition, varies with gestational age at parturition, stage of lactation and nutrient intake of the mother. ${ }^{20}$ At the time of our study all infant formulas contained LA and ALA, in higher amounts than found in human milk; in some brands of formula also a small amount of GLA was found. No formula however, except preterm formula, contained LCPs. As a result, infants fed exclusively with formula have to synthesize the LCPs themselves. It is unclear whether formula fed infants can synthesize the LCPs in such extent that they can meet their LCP requirements. ${ }^{21}$ Breast milk, however, contains a wide variety of EFAs and therefore EFAs in breast milk may account for the protective effect against the development of atopic diseases. The possible role of EFAs in atopic diseases will be outlined in the following paragraphs.

\section{ESSENTIAL FATTY ACIDS IN ATOPIC DISEASE; OBSERVATIONAL DATA}

In 1937, decreased levels of AA were reported in the serum of children with $A D .{ }^{22}$ More recent studies have also shown abnormal $n-6$ fatty acid levels in patients with AD: decreased levels of n-6 LCPs in plasma phospholipids, red blood cells and mononuclear cells of patients. ${ }^{23,24}$ Studies also showed increased levels of LA and an increased ratio of LA compared to its derivatives in plasma and in adipose tissue. ${ }^{25}$

A study from the early 1990 s by Galli et al. showed that changes in fatty acid profiles preceded the development of $A D$ in infants ${ }^{26}$ and thus presented evidence of a possible causal relationship. They compared the EFA status of 13 infants who developed atopy to non-atopic intants in 57 new-borns 'at risk' of atopic disease. The atopic infants had lower 
Figure 1. The main pathways of fatty acid synthesis and conversion

n-6 family

Diet

18:2n-6

linoleic acid

(LA)<smiles>C=[AlH2]</smiles>

18:3n-6

gamma-linolenic acid

(GLA)

cyclo-oxygenase 20:3n-6

elongation

n-3 family

Diet

18:3n-3

alpha-linolenic acid delta- 6 desaturation

(ALA)

on

18:4n-3

stearidonic acid
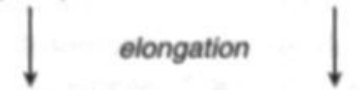

$20: 4 n-3$

1 -series PGs $\longleftarrow$ dihomo-gamma linolenic acid

(DGLA)

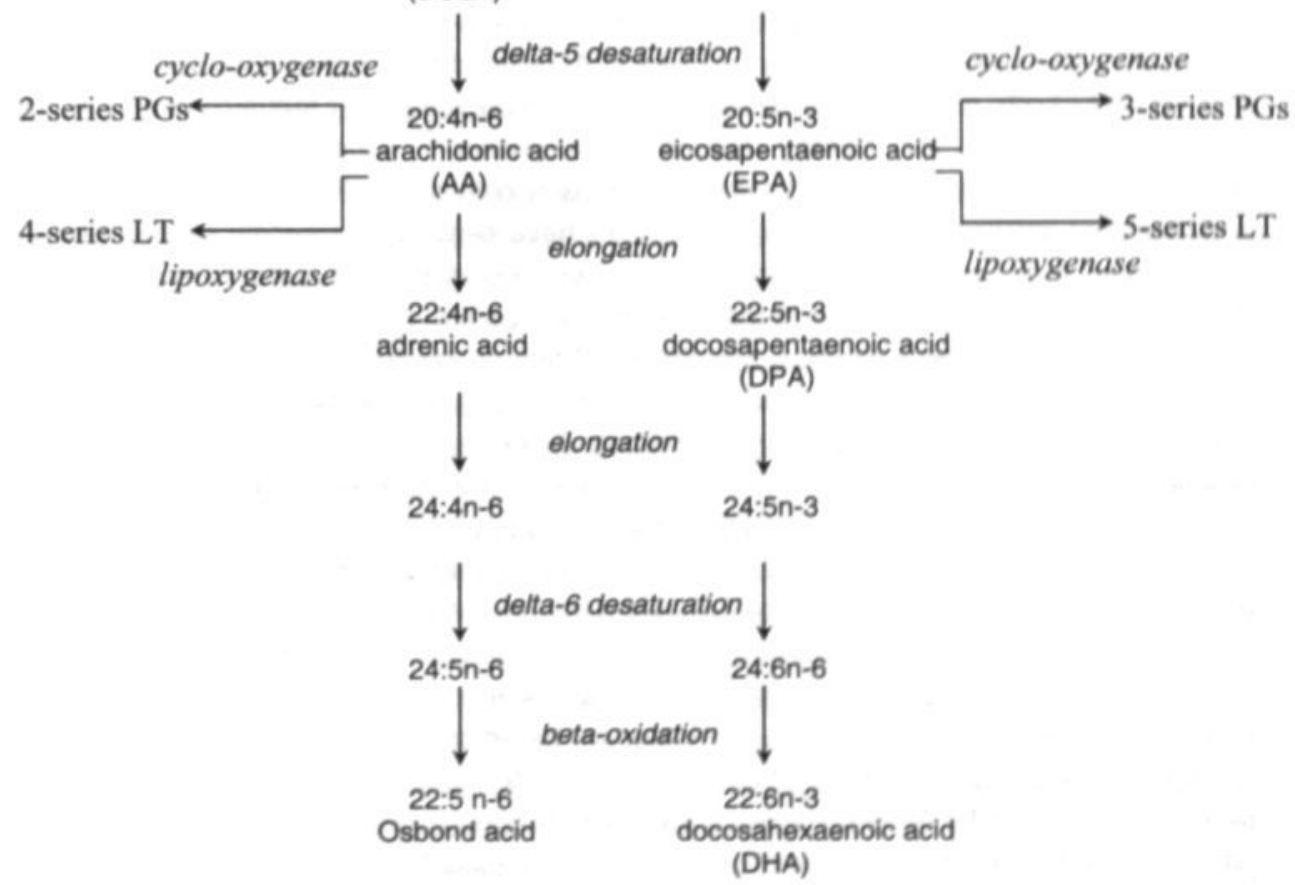

$P G=$ prostaglandine, $\mathrm{LT}=$ leukotriene 
levels of DGLA and AA as well as a lower AALA ratio in cord blood plasma phospholipids and also in plasma phospholipids at the age of 1 and 3 months, but not anymore at 6 months. This suggested a role for essential fatty acids in the early stage of the development of atopy. A causal role of low EFA supply in the etiology of AD was also suggested by studies of Wright and Bolton ${ }^{27}$ and by Businco et al. ${ }^{28}$ who reported lower levels of LA and lower DHA levels in breast milk from mothers whose infants developed AD. LA and ALA levels were not lower or even slightly higher.

During the 1980s the delta-6-desaturase hypothesis was postulated to explain the findings of the observational data. An atopic constitution is associated with a reduced conversion of linoleic acid to its derivatives, possibly caused by an impaired activity of the enzyme delta-6desaturase (D6D), resulting in higher levels of LA and lower levels of GLA and its LCPs. Because DGLA is also lower, the levels of its product prostaglandine E1 (PGE1) are lower too. It was hypothesized that this lower level of PGE1 had several immunomodulating properties and thus could lead to AD. First of all, PGE1 has a relative anti-inflammatory effect by inhibiting the formation of the pro-inflammatory PGE2 from AA (Figure 1). It was also suggested that PGE1 enhanced T Iymphocyte differentiation and maturation. ${ }^{29.30}$ Manku et al. ${ }^{24}$ reported a significant elevation of PGE1 plasma levels after supplementation of $A D$ patients with GLA.

Some of the studies mentioned above showed also abnormalities in $n-3$ fatty acid levels, ${ }^{31}$ but these findings were less consistent than those of the $n-6$ fatty acids and initially ignored.

During the early 1990s the attention for EFAs in AD shifted from n-6 EFAs solely to n-6 EFAs and $n-3$ EFAs both, and the balance between them. It became clear that also dietary n-3 fatty acids are essential for healthy human development, ${ }^{32}$ especially retinal and brain development. Several studies indicated that diets rich in n-3 PUFAs are anti-inflammatory and immunosuppressive in vivo, although there have been relatively few studies in man. ${ }^{33}$ The prostaglandines produced from n-3 EFAs are less pro-inflammatory than PGE2, and EPA (20:5n-3) inhibits the formation of PGE2. Lately reviews on changes in EFA consumption have been published. Simopoulos ${ }^{34}$ has suggested that humans have evolved on a diet that contained about equal amounts of $n-3$ and $n-6$ EFAs. In the current Western diet, however, the ratio between $n-3$ and n-6 EFAs is 1:20-30. ${ }^{35}$ Because of this diet, production of n-6 EFAs are formed in larger quantities than those formed from n-3 EFAs. Black and Sharpe ${ }^{36}$ were the first to relate the changes and differences in EFA consumption with changes in the prevalence of asthma in the last decades in developing countries. They postulated the hypothesis that the lower $n-3 / n-6$ ratio in the diet might explain the increase of asthma, eczema and allergic rhinitis. Because nutrients in the diet are correlated with each other, observational studies of dietary intake cannot produce proof for a cause and effect relationship with disease outcomes. For instance, the decrease of the ratio between n-6 and $n-3$ fatty acids in human consumption, as result of the increase of $n-6$ fatty acids, has also been accompanied by an simultaneous increase in consumption of trans fatty acids. ${ }^{34}$ It is difficult to determine from observational, population based studies whether the observed associations between the increase of atopic diseases and EFA consumption are isolated or that trans fatty acids play a role in this. To assess a relationship between one (group of) EFA(s) and atopic diseases, observational studies should determine EFA intake in individuals and link these intakes with atopic diseases. Nutritional observational studies like these can be done, if EFA composition of nutrients could be estimated accurately. As this is 
not the case yet, the best studies to provide the most persuasive evidence of a cause and effect relationship are randomized controlled clinical trials (RCTs).

\section{ESSENTIAL FATTY ACIDS: EXPERIMENTAL DATA}

Because the conversion of LA to GLA seems to be the rate-limiting step in the total series of conversions, supplementation of GLA may circumvent this enzyme system and prevent atopic disease or decrease its severity. The results of early trials suggested that supplementing patients with GLA (evening primrose oil, borage oil or black current seed oil) could decrease the severity of AD. ${ }^{37.36}$ This was confirmed by the first meta-analysis of nine placebo-controlled RCTs of evening primrose oil, including seven small, unpublished studies. ${ }^{30}$ However, controversy was raised over the meta-analysis because the data of the unpublished studies were never made available in the public domain. ${ }^{40}$ Since the publication of this meta-analysis more trials have been performed with conflicting results. The first supplementation trials with n-3 EFAs to alleviate AD took place in the late eighties and nineties, ${ }^{41-44}$ but the main focus remained on GLA.

The first trials showed promising results and many trials have been published since. All were done in patients with $A D$ but none had addressed the preventive effect of GLA on AD so far.

\section{THE ROLE OF BREAST-FEEDING}

In a meta-analysis Gdalevich recently has shown that exclusive breast-feeding during the first three months of life is associated with a lower incidence rate of AD during childhood, in particular in children with a family history of atopy. ${ }^{45}$ Saarinen showed in a cohort study that breast-feeding protects against the development of atopic diseases, even until adolescence. ${ }^{46}$ Breast milk contains a multitude of components that may provide immune protection to the breast-fed infant and that also may promote development of neonatal immune competence, such as immunoglobulines, hormones, growth factors, fatty acids and antioxidants. ${ }^{47}$ However, the levels of these protective factors differ between mothers, depending on their immune status and their diet and this may influence the ability to reduce the risk of atopic disease. ${ }^{47}$ Breast-feeding has a place in the essential fatty acid hypothesis. Wright and Bolton ${ }^{27}$ and Businco ${ }^{28}$ found that mothers breast-feeding children with eczema had higher concentrations of LA and lower concentrations of its LCPs in their milk, compared to mothers with healthy children. These findings are consistent with the hypothesis that development of atopic dermatitis is related to an impaired D6D activity. Other studies have shown that the breast milk composition of atopic mothers differs from non-atopic mothers in the same way. ${ }^{48}$ If the D6D-hypothesis is true, infants with impaired D6D activity would rely more heavily on exogenous LCP supply, and would therefore be at higher risk of developing atopic disease when they are fed formula without LCPs, or when they are breast-fed by an atopic mother who would also have lower LCPs in her breast milk as an inherent part of her atopic constitution. ${ }^{49}$ 


\section{OTHER HYPOTHESES FOR THE INCREASE OF ATOPY}

One of the most popular hypotheses these days for the rising trend in atopic diseases is the so-called hygiene-hypothesis coined by Strachan in $1989 .^{50} \mathrm{He}$ had found that $A D$ and haytever were negatively related to the number of older children in the house (the siblingeffect). He suggested that allergic diseases were prevented by infection in early childhood, transmitted by contact with older siblings. He also suggested that in the past century declining family size, improvements in housing conditions, and higher standards of personal cleanliness had reduced the opportunity for cross infections in young families. Lack of immune stimulation may have resulted in a more widespread clinical expression of atopic disease. Some epidemiological studies have shown that the risk of atopy is higher in children who did not raise an immune response against measles, hepatitis $A$ and tuberculosis. ${ }^{51-53}$ Another hypothesis is that the gut flora is involved in the development of atopic diseases. This association has been based on findings of differences in the gut flora between children with and without atopic diseases. ${ }^{54-56}$ It has been shown that these differences precede the development of atopic diseases. Breast milk also facilitates the establishment of a normal gut flora that inhibits colonization by pathogens and stimulates the growth of beneficial microorganisms. ${ }^{57}$ As the microbial gut flora drives the maturation of the immune system, its composition may play a role in the development of and protection against atopic diseases. ${ }^{58}$

\section{AIM AND OUTLINE OF THE THESIS}

The aim of this thesis is to evaluate the role of essential fatty acids in the development of AD in early life. Reasons to focus on early life are based on the following observations; 1) the EFA-status of newborns and exclusively breast-fed infants is entirely dependent on the mother; ${ }^{15}$ 2) mothers with atopic children have an aberrant fatty acid profile in their breast milk compared to mothers of non-atopic infants, ${ }^{27,28} 3$ ) children who develop atopic symptoms have a similar aberration of their fatty acid profile in cord blood and blood in the first months of life; ${ }^{26} 4$ ) the possible role of essential fatty acid derived prostaglandins on the maturation of the immune system. ${ }^{59}$

We performed two studies; the first study is an observational study in a group of breast-fed infants. In this study we bear further on the study of Galli et al., who showed that differences in $n-6$ fatty acids preceded the development of $A D .^{26}$ We studied the relation between perinatal EFA status in mother and infant and the development of $A D$ in infants at the age of one year (chapter two).

The second study is a randomized controlled trial in formula-fed infants. This trial was intended to evaluate the role of dietary n-6 EFAs in the early development of atopy, and to test the hypothesis of an impaired activity of D6D. To circumvent the first (supposedly rate limiting) conversion from LA to GLA we supplemented the diet of the infants for six months with GLA from borage oil (chapter three).

After the data for these studies were collected, a paper was published suggesting that the sibling effect was possibly mediated in utero already, instead of in early infancy. The authors had shown that IgE levels in cord blood were lower if the infant had an older sibling compared to if it was the first-born infant. ${ }^{60}$ This points to a uterine influence of earlier pregnancies (or parity). As it has been shown that maternal EFA status might be depleted 
after multiple pregnancies ${ }^{19}$ we evaluated whether the effect of parity on neonatal IgE could also be shown in our data, and which role EFAs play in this relation (chapter four).

Finally, we performed an updated meta-analysis of trials of EFA supplementation in patients with $A D$, because the former was done in 1989 and many more studies have been performed since (chapter five).

\section{REFERENCES}

1. Worldwide variation in prevalence of symptoms of asthma, allergic minoconjunctivitis, and atopic eczema: ISAAC. The International Study of Asthma and Allergies in Childhood (ISAAC) Steering Committee. Lancet. 1998;351:1225-32.

2. Strannegard $\mathrm{O}$, Strannegard IL. The causes of the increasing prevalence of allergy: is atopy a microbial deprivation disorden Allergy. 2001;56:91-102.

3. Coca AF, Cooke RC. On the classification of the phenomena of hypersensitiveness. J Immunol. 1922;8:163-182.

4. Johansson SG, Hourihane JO, Bousquet J, et al. A revised nomenclature for allergy: An EAACI position statement from the EAACI nomenclature task force. Allergy. 2001:56:813-824.

5. O'Loughlin S, Diaz-Perez JL, Gleich GJ, et al. Serum IgE in dermatitis and dermatosis: an analysis of 497 cases. Arch Dermatol. 1977;113:309-15.

6. Ownby DR. Clinical significance of Immunoglobulin E. In: Middleton E, ed. Allergy; Principles and practice. St. Louis: Mosby, 1992:770-782.

7. Negrao-Correa D. Importance of immunoglobulin $\mathrm{E}(\mathrm{ggE})$ in the protective mechanism against gastrointestinal nematode infection: looking at the intestinal mucosae. Revista do Instituto de Medicina Tropical de Săo Paulo 2001;43:291-9.

8. Archer CB. The pathophysiology and clinical features of atopic dermatitis. In: Williams H, ed. Atopic dermatitis; The Epidemiology, Causes and Prevention of Atopic Eczema. Cambridge: Cambridge University Press, 2000.

9. Zeiger RS, Heller S. The development and prediction of atopy in high-risk children: follow-up at age seven years in a prospective randomized study of combined maternal and infant food allergen avoidance. J Allergy Clin Immunol. 1995;95:1179-90.

10. Hanifin JM, Rajka G. Diagnostic features of atopic dermatitis. Acta Derm Venereol.Suppl. 1980;92:44-47.

11. Williams HC, Bumey PG, Strachan D, et al. The U.K. Working Party's Diagnostic Criteria for Atopic Dermatitis. II. Observer variation of clinical diagnosis and signs of atopic dermatitis. $\mathrm{Br} J$ Dermatol. 1994;131:397-405.

12. Williams HC, Burney PG, Pembroke AC, et al. The U.K. Working Party's Diagnostic Criteria for Atopic Dermatitis. III. Independent hospital validation. Br J Dermatol. 1994:131:406-16.

13. Williams HC, Bumey PG, Hay RJ, et al. The U.K. Working Party's Diagnostic Criteria for Atopic Dermatitis. I. Derivation of a minimum set of discriminators for atopic dermatitis. Br J Dermatol. 1994:131:383-96.

14. Holman R. Nutritional and metabolic interrelationships between fatty acids. Fed Proc. 1964;23:1062-7.

15. Hornstra G. Essential fatty acids in mothers and their neonates. J Clin Nutr 2000;71:1262s-9s.

16. Brenner RR. Regulatory function of delta6 desaturate - key enzyme of polyunsaturated fatty acid synthesis. Adv Exp Med Biol. 1977;83:85-101.

17. Neuringer M, Connor WE, Lin DS, et al. Biochemical and functional effects of prenatal and postnatal omega 3 fatty acid deficiency on retina and brain in mesus monkeys. Proceedings of the National Academy of Sciences of the United States of America 1986;83:4021-5.

18. Hoffman DR, Uauy R. Essentiality of dietary omega 3 fatty acids for premature infants: plasma and red blood cell fatty acid composition. Lipids. 1992;27:886-95. 
19. Al MD, van Houwelingen AC, Hornstra G. Relation between birth order and the maternal and neonatal docosahexaenoic acid status. Eur J Clin Nutr. 1997;51:548-53.

20. Jensen RG. Handbook of Milk Composition. San Diego: Academic Press, 1995.

21. Innis SM, Sprecher H, Hachey D, et al. Neonatal polyunsaturated fatty acid metabolism. Lipids. 1999:34:139-49.

22. Brown WR, Hansen AE. Arachidonic and Linolic Acid of the serum in normal and eczematous human subjects. Proc Soc Exp Biol Med. 1937;36:113-117.

23. Lindskov R, Holmer G. Polyunsaturated fatty acids in plasma, red blood cells and mononuclear cell phospholipids of patients with atopic dermatitis. Allergy. 1992;47:517-21.

24. Manku MS, Horrobin DF, Morse N, et al. Reduced levels of prostaglandin precursors in the blood of atopic patients: defective delta-6-desaturase function as a biochemical basis for atopy. Prostaglandins Leukot Med. 1982;9:615-28.

25. Wright S, Sanders TA. Adipose tissue essential fatty acid composition in patients with atopic eczema. Eur J Clin Nutr. 1991;45:501-5.

26. Galli E, Picardo M, Chini L, et al. Analysis of polyunsaturated fatty acids in newborn sera: a screening tool for atopic disease? Br J Dermatol. 1994;130:752-6.

27. Wright $\mathrm{S}$, Bolton $\mathrm{C}$. Breast milk fatty acids in mothers of children with atopic eczema. $\mathrm{Br} \mathrm{J}$ Nutr. 1989;62:693-7.

28. Businco L, loppi M, Morse NL, et al. Breast milk from mothers of children with newly developed atopic eczema has low levels of long chain polyunsaturated fatty acids. J Allergy Clin Immunol. 1993:91:1134-9.

29. Melnik BC, Plewig G. Is the origin of atopy linked to deficient conversion of omega-6-fatty acids to prostaglandin E1? [see comments]. J Am Acad Dermatol. 1989;21:557-63.

30. Melnik B. Plewig G. Are disturbances of omega-6-fatty acid metabolism involved in the pathogenesis of atopic dermatitis? Acta Dermato Venereologica - Suppl. 1992;176:77-85.

31. Manku MS. Horrobin DF, Morse NL, et al. Essential fatty acids in the plasma phospholipids of patients with atopic eczema. Br J Dermatol. 1984;110:643-8.

32. Bjerve KS. Omega 3 fatty acid deficiency in man: implications for the requirement of alphalinolenic acid and long-chain omega 3 fatty acids. World Rev Nutr Diet. 1991;66:133-42.

33. Calder PC. N-3 polyunsaturated fatty acids and immune cell function. Adv Enzyme Regul. 1997;37:197-237.

34. Simopoulos AP. Essential fatty acids in health and chronic disease. Am J Clin Nutr 1999;70:560s$569 \mathrm{~s}$.

35. Hulshof KF, van Erp Baart MA, Anttolainen M, et al. Intake of fatty acids in western Europe with emphasis on trans fatty acids: the TRANSFAIR Study. Eur J Clin Nutr. 1999;53:143-57.

36. Black PN, Sharpe S. Dietary fat and asthma: is there a connection? Eur Respir J. 1997;10:6-12.

37. Lovell CR, Burton JL, Horrobin DF. Treatment of atopic eczema with evening primrose oil. Lancet 1981:1:278.

38. Wright S, Burton JL. Oral evening-primrose-seed oil improves atopic eczema. Lancet 1982;2:1120-2.

39. Morse PF, Horrobin DF, Manku MS, et al. Meta-analysis of placebo-controlled studies of the efficacy of Epogam in the treatment of atopic eczema. Relationship between plasma essential fatty acid changes and clinical response. Br J Dermatol. 1989;121:75-90.

40. Hoare C, Li Wan Po A, Williams H. Systematic review of treatments for atopic eczema. Health Technol Assess. 2000;4:1-191.

41. Bjorneboe A, Soyland E, Bjorneboe GE, et al. Effect of $n-3$ fatty acid supplement to patients with atopic dermatitis. J Intern Med. Suppl. - 1989;225:233-6.

42. Berth Jones J, Graham Brown RAC. Placebo-controlled trial of essential fatty acid supplementation in atopic dermatitis. Lancet. 1993;341:1557-1560.

43. Soyland E, Funk J, Rajka G, et al. Dietary supplementation with very long-chain $n-3$ fatty acids in patients with atopic dermatitis. A double-blind, multicentre study. Br J Dermatol. 1994;130:757-64.

44. Gimenez Arnau A, Barranco C, Alberola M, et al. Effects of linoleic acid supplements on atopic dermatitis. Adv Exp Med Biol. 1997:433285-9. 
45. Gdalevich M, Mimouni D, David M, Mimouni M. Breast-feeding and the onset of atopic dermatitis in childhood: a systematic review and meta-analysis of prospective studies. J Am Acad Dermatol. 2001;45:520-7.

46. Saarinen UM, Kajosaari M. Breastfeeding as prophylaxis against atopic disease: prospective follow-up study until 17 years old [see comments]. Lancet 1995:346:1065-9.

47. Hoppu U, Kalliomaki M, Laiho $K$, et al. Breast milk-immunomodulatory signals against allergic diseases. Allergy. 2001;56:23-6.

48. Thijs C, Houwelingen A, Poorterman I, et al. Essential fatty acids in breast milk of atopic mothers: comparison with non-atopic mothers, and effect of borage oil supplementation. Eur $\mathrm{J}$ Clin Nutr. 2000:54:234-8.

49. Meinik BC. [A chance for the prevention of atopic diseases]. Monatsschr Kinderheilkd. 1990;138:162-6

50. Strachan DP. Hay fever, hygiene, and household size. BMJ. 1989;299:1259-60.

51. Matricardi PM, Rosmini F, Ferrigno L, et al. Cross sectional retrospective study of prevalence of atopy among Italian military students with antibodies against hepatitis A virus. BMJ. 1997:314:9991003.

52. Shaheen SO, Aaby P. Hall AJ, et al. Measles and atopy in Guinea-Bissau. Lancet. 1996;347:1792-6.

53. Shirakawa T, Enomoto T, Shimazu S, et al. The inverse association between tuberculin responses and atopic disorder. Science. 1997;275:77-9.

54. Sepp E, Julge K, Vasar M, et al. Intestinal microflora of Estonian and Swedish infants. Acta Paediatr Scand. 1997;86:956-61.

55. Bjorksten B, Sepp E, Julge K, et al Allergy development and the intestinal microflora during the first year of life. J Allergy Clin Immunol. 2001;108:516-20.

56. Bjorksten B, Naaber P, Sepp E, et al. The intestinal microflora in allergic Estonian and Swedish 2. year-old children. Clin Exp Allergy. 1999;29:342-6.

57. Dai D, Walker WA. Protective nutrients and bacterial colonization in the immature human gut. Adv Pediatr.1999;46:353-82.

58. Bjorksten B. Environment and infant immunity. Proc Nutr Soc. 1999;58:729-32.

59. Melnik B, Plewig G. Essential fatty acids, eicosanoids and postnatal T-cell maturation implications for treatment and prevention of atopy. J Dermatolog Treat. 1994:5:157-161.

60. Karmaus W, Arshad H, Mattes J. Does the sibling effect have its origin in utero? Investigating birth order, cord blood immunoglobulin $\mathrm{E}$ concentration, and allergic sensitization at age 4 years. Am J Epidemiol. 2001:154:909-15. 



\title{
Perinatal essential fatty acid status and the development of atopy at age one year - the EFAtop study
}

\author{
Christel J.A.W. van Gool' \\ Carel Thijs' \\ Adriana C. v. Houwelingen ${ }^{2}$ \\ Charles J.M. Henquet' \\ Pieter C. Dagnelie \\ Jaap Schrander ${ }^{4}$ \\ Paul P.C.A. Menheeres \\ Piet A. van den Brandt ${ }^{\prime}$
}

'Department of Epidemiology. Maastricht University 2Department of Human Biology, Maastricht University 'Department of Dermatology, Maastricht University

'Department of Pediatrics, Maastricht University

sDepartment of Clinical Chemistry, University Hospital Masstricht 


\begin{abstract}
OBJECTTVE

The relationship between essential fatty acid (EFA) status and the occurrence of atopic dermatitis has been explained by two hypotheses; 1) a reduced activity of the delta-6-desaturase (D6D) enzyme system, 2) an increased dietary intake of n-6 fatty acids relative to n- 3 fatty acids.
\end{abstract}

\title{
DESIGN
}

A prospective study to test these hypotheses by investigating the role of EFAs in 64 atopic mothers and their infants in relation to the development of atopic dermatitis. EFA status was determined in maternal plasma phospholipids prepartum, in breast milk fat at one and four weeks postpartum, and in plasma phospholipids of the infant at age one week, three months, six months and one year. Metabolic indices for D6D activity; $n-6 / n-3$ ratio; and deficiency indices were calculated from the fatty acid levels. Outcome measures at age one year were: occurrence of atopic dermatitis (AD, according to UK Working Party); severity of atopic dermatitis (SCORAD); and total serum $\lg \mathrm{E}$.

\section{RESULTS}

In the infants with the highest vs. those with the lowest tertile of the SCORAD, the docosahexaenoic acid $(22: 6 n-3)$ sufficiency index was $53 \%$ lower in mothers prepartum $(P<0.03), 31 \%$ lower in the infant at one week $(P=0.01)$ and $58 \%$ lower at three months $(P=0.02)$. Metabolic indices and $n-6 / n-3$ ratio were not associated with $A D, S C O R A D$ or $\lg E$.

\section{CONCLUSION}

The results do not confirm the D6D hypothesis. They suggest that severity of atopic dermatitis at age one year is related to reduced levels of individual n-3 fatty acids, in particular a relative shortage of docosahexaenoic acid (22:6n-3) supply over the first three months of life. This is consistent with the hypothesis that the rise of atopic dermatitis in developed countries is due to a shortage of dietary intake of n-3 EFA relative to n-6 EFA intake. 


\section{INTRODUCTION}

Linoleic acid (LA, 18:2n-6) and alpha-linolenic acid (ALA, 18:3n-3) need to be consumed with the diet, as they cannot be synthesized de novo by humans.' They are the first fatty acids (FA), competing for the same enzyme systems, in a chain of more unsaturated and elongated products, the so-called long chain polyenes (LCPs). LCPs are important structural and functional membrane components. Some of these LCPs are also precursors for prostanoids and eicosanoids. Even though some experts in the field restrict the definition of essential fatty acids (EFAs) to LA and ALA alone, others define EFAs as LA, ALA and their LCPs together. In this paper we will use the latter definition.

In 1937, Brown and Hansen reported lower levels of arachidonic acid (AA, 20:4n-6) in infants with atopic dermatitis, and suggested a defective EFA metabolism to be the causal factor. ${ }^{2}$ Increased levels of LA and substantially lower levels of its LCPs, such as AA, were observed in blood, ${ }^{3-5}$ adipose tissue ${ }^{4}$ and breast milk $^{6}$ of adults with atopic disease, as compared to healthy subjects. It was suggested that these fatty acid (FA) profiles in subjects with atopic dermatitis reflect a lower activity of delta-6-desaturase (D6D), the enzyme determining the rate-limiting step in the desaturase-elongase pathway of EFAs. ${ }^{3.7}$

In the last decade, further evidence supporting the hypothesis that an impaired D6D activity contributes to the development of atopic dermatitis came from a prospective study by Galli and co-workers. This study showed that low n-6 LCP levels preceded the development of atopic dermatitis in infants, ${ }^{8}$ suggesting a causal relationship. The authors demonstrated that the concentrations of dihomo-gamma-linolenic acid (DGLA, 20:3n-6) and AA, both LCPs of $n-6$ fatty acids, were consistently and significantly lower in umbilical cord blood and in serum at one and three months of age in infants who developed atopy at the age of one year compared to infants who remained unaffected.

There are indications that breast-feeding may protect infants at risk from developing atopic dermatitis. ${ }^{9.10}$ One of the differences between breast milk and infant formula is that breast milk, in contrast with most current infant formulas, contains LCPs. In studies by Wright and Bolton" and by Businco et al. ${ }^{12}$ breast milk from mothers whose infants subsequently developed $A D$ contained $14-50 \%$ less $n-6$ LCPs than milk from mothers whose infants remained unaffected. These findings are consistent with the hypothesis that development of atopic dermatitis is related to an impaired D6D activity. If this hypothesis is true, infants with impaired D6D activity would rely more heavily on exogenous LCP supply, and would therefore be at higher risk of developing atopic disease when they are fed formula without LCPs, or are breastfed by an atopic mother who would also have lower LCPs in her breast milk as an inherent part of her atopic constitution. ${ }^{13}$

In the 1990's, a second hypothesis emerged on the role of EFAs explaining the gradual increase of atopic diseases in the preceding decades. Based on studies of the increased prevalence of atopic diseases and studies of trends in the consumption of FA in developed countries, it was noted that the increased consumption of vegetable oils rich in LA and a simultaneously reduced consumption of fatty fish (rich in n-3 EFAs) went together with a concurrent increase of the prevalence of atopic disease. ${ }^{14,15}$ It was hypothesized that an excessive consumption of LA and a subsequent increase in ratio between n-6 and n-3 EFAs in the diet may lead to atopic disease.

The aim of the present study was to evaluate the following hypotheses; 1) an impaired D6D activity in infants and/or their mothers is related to development of atopy, and 2) an 
imbalance in the ratio between dietary n-6 and n-3 EFAs is related to development of atopy. More specifically, we wanted to evaluate, in a prospective, observational design, the relation between maternal and infant EFA status and the development of atopic dermatitis and IgE in infants in the first year of life. Fatty acid values of plasma phospholipids and in total lipid of breast milk were used to calculate indices of D6D activity and of dietary $n-6 / n-3$ balance. It has been shown that EFAs of plasma phospholipids mirror dietary EFA intake in infants and adolescents $^{16}$ and that changes in the intake of EFAs by lactating mothers are partially reflected in the EFA composition of breast milk. ${ }^{6}$

\section{MATERIALS AND METHODS}

\section{STUDY DESIGN AND POPULATION}

This study is a prospective, observational study in children at risk for developing atopic dermatitis. Subjects eligible for the study were atopic pregnant women, intending to breastfeed their infant. The atopic status of both parents was assessed using a telephone questionnaire, developed and validated earlier (Van Bokhoven et al., 1996, unpublished). Inclusion criteria for atopic mothers were a history of allergic asthma or rhinitis (defined as a combination of: at least two of a list of asthma and rhinitis complaints; related to exposure to pets, house dust or pollen or to blossoming of certain trees or grass; and onset before age $16 \mathrm{y}$ ), or atopic dermatitis (defined as: typical localization and onset before age $6 \mathrm{y}$ ), or history of positive allergy test, or improvement of astma or rhinitis complaints with use of antihistamine drugs. Exclusion criteria were: diabetes treated with medication and/or a diet, (pre-)eclampsia and metabolic diseases. Women were recruited by midwives and by advertisements in local newspapers.

Inclusion criteria for the infants were: gestational age of at least 38 weeks, birth weight > 2500 grams, an uncomplicated perinatal period, and exclusive breast-feeding for at least 4 weeks. All data were collected at the participants' homes except for the final visit at the age of one year, which took place at the University Hospital Maastricht, The Netherlands. The medical ethics committee of the University Hospital Maastricht approved the study and written informed consent was obtained from both parents.

\section{SAMPLING}

Blood was collected from the mothers by venepuncture of the median cubital vein at 34-36 weeks of gestation; from the infants at the age of one week, three months and 6 months by heel prick or finger prick and at the age of one year by venepuncture of the hand vein or of the median cubital vein. Blood and was collected in EDTA-containing tubes (Becton Dickinson, NJ USA) and serum separator tubes (Sherwood-Davis \& Geck, StLouis, USA). The samples were transported on ice/water mixture, and within 24 hours after collection plasma and serum were separated from erythrocytes by centrifugation $\left(3000 \mathrm{G}\right.$ at $4^{\circ} \mathrm{C}$, during 10 minutes) and stored at $-20^{\circ} \mathrm{C}$ (serum) or $-50^{\circ} \mathrm{C}$ (plasma) under nitrogen until analysis. 
Breast milk samples were collected at one week and at four weeks postpartum. These samples were foremilk collected by the mothers at home between 9.00 am and $3.00 \mathrm{pm}$, after their infant had finished drinking from the contra-lateral breast.

Milk samples were preserved by mixing approximately $2 \mathrm{ml}$ of milk with $2 \mu \mathrm{l}$ methanol (Merck, Darmstadt, Germany) containing $500 \mathrm{ml}$ of butylated hydroxytoluene (Sigma, Zwijndrecht, the Netherlands) per liter, transported on ice/water mixture and stored at $-50^{\circ} \mathrm{C}$ until analysis.

\section{LABORATORY ANALYSES}

\section{FATTY ACID ANALYSIS}

Fatty acids (FAs) were analysed as previously described." ${ }^{17}$ Lipid extracts were prepared from plasma samples, and phospholipid (PL) fractions separated using aminopropyl-bonded phase columns. ${ }^{18}$ PLs were hydrolysed and FAs transmethylated with boron trifluoride (SIGMA) in methanol. The composition of the obtained FA methyl esters was determined by capillary gas chromatography using a polar capillary column (CPSil 88; Chrompack, Middelburg, the Netherlands), with helium as carrier gas. The amount of each FA was quantified by adding to each sample an internal standard (dinonadecanoyl [19:0]phosphatidylcholine, SIGMA). Results are reported as weight percentage (wt\%) of total FAs. computed as previously described. ${ }^{10}$

Based on a study by Businco, ${ }^{12}$ in which the ratio between the parent EFAs and their substrates was used as an indication of $\mathrm{D} 6 \mathrm{D}$ activity, two metabolic indices were defined for the $n-6$ and $n-3$ EFAs separately as:

Metabolic index n-6 EFAs $=[(18: 3 n-6+20: 3 n-6+20: 4 n-6+22: 4 n-6+22: 5 n-6) /(18: 2 n-6)]$

Metabolic index $n-3$ EFAs $=[(18: 4 n-3+20: 5 n-3+22: 5 n-3+22: 6 n-3) /(18: 3 n-3)]$

Furthermore, four functional status markers of EFAs were assessed. First, whenever the diet is deficient in LA or ALA or their LCPs, conversion of 18:1n-9 is increased, through the same desaturation and elongation systems as the EFAs, consequently increasing the levels of $20: 3 n-9$. The latter is, therefore, a negative deficiency marker for total $n-6$ and n-3 EFA (Homstra, 2000). Second, in case the dietary supply of docosahexaenoic acid (DHA, 22:6n3 ) is insufficient, the conversion of $22: 4 n-6$ to $22: 5 n-6$ increases, resulting in relatively high levels of 22:5n-6. The latter is therefore a deficiency marker for low dietary DHA supply. Third and fourth, two existing indices were used to indicate DHA status: the DHA deficiency index, 22:5n-6/22:4n-6, and the DHA sufficiency index, 22:6n-3/22:5n-6. ${ }^{1.2021}$

\section{TOTAL IGE ANALYSES AND SPECIFIC TESTS}

Screening tests for common aero- and food allergens (Phadiatop and Fx5, respectively) and total $\mathrm{lgE}$ were determined in serum from the infants at one year of age. The analysis of these tests has been done using the Pharmacia Unicap system (Pharmacia Upjohn, Upsala, Sweden) as described elsewhere. ${ }^{22}$

If an insufficient amount of venous blood was acquired, finger prick blood was collected on blotting paper. Measurement of total $\mathrm{IgE}$ in eluded blood spot material was then performed in a sandwich assay, as previously described, ${ }^{23}$ with minor modifications; a mixture of anti- 
human IgE monocional antibodies was coupled to Sepharose 4B for catching of IgE; for detection of Sepharose-bound $\mathrm{IgE}$ radio-labelled antibodies against human $\lg \mathrm{E}$, raised in sheep, were used.

\section{DIAGNOSIS OF ATOPIC DERMATTTIS}

At the follow-up visit at the hospital at the age of one year, a trained dermatologist $(\mathrm{CH})$ determined the clinical diagnosis of atopic dermatitis using the UK Working Party criteria. ${ }^{24.25}$ Briefly, the probability of presence of atopic dermatitis is derived from the presence of four clinical symptoms i.e.: (1) presence of itchy rash (PIR, coded as $1=$ present, $0=a b s e n t),(2)$ history or (3) visible flexural dermatitis (HFD or VFD, ibid), and (4) onset before 2 years of age (OB2, ibid). HFD and VFD were modified to the extensor side of the limbs to match the typical clinical predilection sites in infants. ${ }^{24.25}$ The UK-Working Party Probability Score of atopic dermatitis $(A D)$ is then computed as: probability $(A D)=$ odds $(A D) /[0 d d s(A D)+1]$; where odds $(A D)=\exp \left[(4.86+1.84(\mathrm{HFD})+3.46(\mathrm{OB} 2)+2.09(\mathrm{VFD})+1.71(\mathrm{PIR})] .{ }^{24.25}\right.$ Infants with a probability of $A D$ of over 0.50 were considered to have $A D$ in the present study.

Severity of dermatitis was scored by one dermatologist $(\mathrm{CH})$ using the objective clinical criteria of the SCORAD. ${ }^{26,27}$ These criteria include scoring of the involved area with dermatitis (A) and the intensity of the dermatitis (B). Five criteria were used for scoring intensity: erythema, edema/papulation, oozing/crusting, excoriation and lichenification (each with score 0-2). The SCORAD index is computed as: ${ }^{26.27} \mathrm{~A} / 5+7 \mathrm{~B} / 2$.

At the same time the SCORAD was scored independently by one of the investigators (CvG, CT) to assess interobserver variability with the dermatologist.

\section{STATISTICAL ANALYSIS}

Correlation between total serum $\lg E$ and the other outcome measures, were done with Pearson's correlation analyses. Total serum IgE was not normally distributed and was therefore transformed to the natural logarithm IgE.

To compare differences in FA status between infants with high and low values of total IgE or SCORAD, the total group was divided into tertiles of total $\operatorname{lgE}$ or SCORAD and the third (high) tertile was compared to the first (low) tertile. Differences in plasma EFA levels between atopic and non-atopic infants, and between the third and first tertiles of SCORAD and total $\lg \mathrm{E}$, were tested for statistical significance $(\mathrm{a}=0.05$, two-sided). Two-sided $95 \%$ confidence limits (CL) of EFA levels are reported throughout.

\section{RESULTS}

Sixty-five infants, born between December 1997 and November 1998, were included in the study and 64 infants completed follow-up to one year. A history of clinical symptoms of atopic disease was present in all 64 mothers (Table 1) and in 23 fathers. Infants were breastfed for a mean of 198 days (range 35 days - one year), and exclusively breast-fed for a mean of 122 days (range 28 days -294 days). 
Table 1. Characteristics of participating mothers $(n=64)$

\begin{tabular}{|c|c|c|c|}
\hline Atopic history & $n(\%)$ & & \\
\hline atopic asthma & $29(45 \%)$ & & \\
\hline thinitis & $52(81 \%)$ & & \\
\hline atopic dermatitis & $30(47 \%)$ & & \\
\hline positive allergy test in history & $44(69 \%)$ & & \\
\hline use of antihistamine drugs in history & $21(33 \%)$ & & \\
\hline total & $64(100 \%)$ & & \\
\hline \multicolumn{4}{|l|}{ Laboratory measurements } \\
\hline Total serum lgE KU/ & $n \%$ & positive Phadiatop" (n) & positive $F \times 5^{\circ}$ (n) \\
\hline $1-49$ & $21(33 \%)$ & 9 & 0 \\
\hline 50-99 & $15(23 \%)$ & 13 & 0 \\
\hline $100-199$ & $10(15 \%)$ & 8 & 0 \\
\hline $200-3602$ & $18(28 \%)$ & 17 & 8 \\
\hline total & $64(100 \%)$ & 48 & 9 \\
\hline
\end{tabular}

$\mathrm{n}=$ number of participants

Phadiatop $=$ specific inhalant allergy test

Fx5 = tood allergy test directed against cow's milik, egg-white, soy, shell-fish, nuts, wheat

a, b number of participants with positive specific test according to total serum IgE level

At the age of one year, 25 out of 64 infants were diagnosed with AD. All infants with a positive test of specific IgE had a total serum IgE higher than $15 \mathrm{KU} / \mathrm{L}$. All three infants with a positive Phadiatop had also a positive Fx5 test. No correlation was present between total serum IgE (as natural logarithm) and the UK-Working Party probability score $(r=-0.05, P=$ $0.69)$ or between total serum IgE and SCORAD $(r=0.00, P=0.99)$. The correlation between the UK-Working Party probability score and SCORAD was moderate $(r=0.51, P=0.00)$. The correlation between the SCORAD by the dermatologist and the independent investigators was reasonably good $(r=0.74, P=0.01)$. Only the SCORAD of the dermatologist is used in the analyses.

\section{METABOLIC INDICES}

The metabolic index of the n-6 EFAs was not significantly different between infants with and without $A D$, or between infants in the high and low tertiles of $\lg E$, or SCORAD (Table 3). No significant differences in the metabolic index of the n-3 EFAs were observed between infants with and without AD (Table 4). However, in infants with high SCORAD, the metabolic index of the n-3 EFAs was lower at one week, both in breast milk of the mothers $(-38 \%)$ and in plasma of infants $(-26 \%)$ compared to infants with low SCORAD (Table 4). Contradictory to the D6D-hypothesis, breast milk at week four of mothers of infants with high IgE vs. low IgE showed a $65 \%$ higher value of the metabolic index of the n-3 EFAs (Table 4). 
Table 2. Total and specific serum IgE in intants age 1 year $(n=64)$

\begin{tabular}{lcccc}
\hline Total serum lgE KUn & Total $(n(\%))$ & AD $(n)^{\mathrm{a}}$ & positive Phadiatop $(n)^{\mathrm{b}}$ & positive F×5 (n)c \\
\hline low tertile $(0-4.0)$ & $22(34 \%)$ & 12 & 0 & 0 \\
middle tertile $(4.2-15.0)$ & $21(33 \%)$ & 5 & 0 & 0 \\
high tertile $(15.2-136.0)$ & $21(33 \%)$ & 8 & 3 & 9 \\
total & $64(100 \%)$ & 25 & 3 & 9 \\
\hline
\end{tabular}

$\mathrm{n}=$ number of participants

$A D=$ infants diagnosed with atopic dermatitis at the age of 1 year according to UK-Working party probability score of atopic dermatitis (score $>0.50)$

Phadiatop $=$ specific test against aero-allergens

Fx5 = food allergy test against cow's milk, egg-white, soy, shell-fish, nuts, wheat

a, b, c number of participants with positive specific test according to total serum IgE level

Most individual LCPs (results available on request) did not differ between infants with and without AD, or between infants with high and low SCORAD, or IgE. Out of 294 comparisons of individual EFAs, some differences were statistically significant ( $P$-values between 0.02 and 0.05 ), but there was no consistent pattern between different atopic outcomes and no pattern that agreed with the D6D hypothesis (data not shown). For instance, at 34 weeks of gestation in the mothers whose children developed AD, DGLA was $10 \%$ lower $(P=0.03)$, but showed no relation with SCORAD or IgE. At three months of age the difference in DGLA was no longer present, but at this moment GLA was $40 \%$ higher in infants with $A D(P=0.02)$.

\section{N-6/N-3 EFA BALANCE}

The $n-6 / n-3$ ratio was not statistically different at any point of time in plasma of infants and in plasma and milk of mothers according to $A D$, nor according to the $\lg E$ tertiles. An $18 \%$ higher $n-6 / n-3$ ratio was observed only pre partum in the mothers of the infants with high vs. Iow SCORAD, but there was no consistent gradient over the tertiles (Table 5).

With respect to individual n-3 LCPs, significant differences among 105 comparisons were always in the direction of lower n-3 LCP concentrations when comparing infants with vs. without $A D$ and high vs. low SCORAD. Differences between infants with vs. without $A D$ were present in: the mothers' breast milk at 4 weeks postpartum $(47 \%$ lower $20: 5 n-3 ; P=0.04)$. Differences between infants with high vs. Iow SCORAD were present during pregnancy (12\% lower $22: 5 n-3 ; P=0.04)$, in breast milk at one week $(29 \%$ lower $22: 5 n-3 ; P=0.004$ and $28 \%$ lower $22: 6 n-3 ; P=0.01)$ and in breast milk at 4 weeks $(25 \%$ lower $22: 5 n-3 ; P=$ 0.01 ). In these cases there was always a gradient over the tertiles. 
Table 3 Perinatal $n-6$ metabolic indexe related to atopic outcomes in the intant at age 1 year

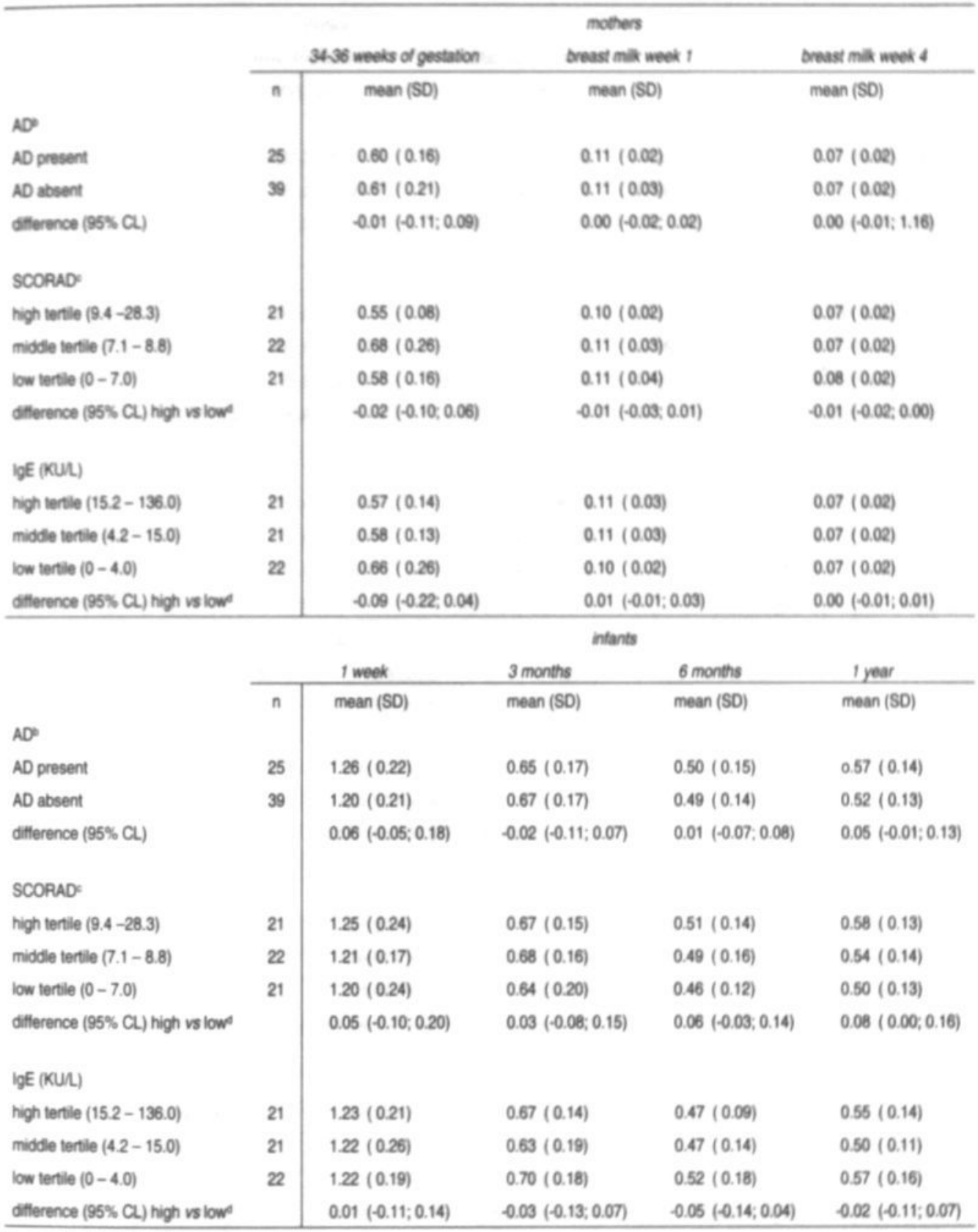

\footnotetext{
$n=$ number of participants
}

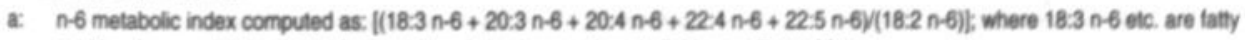
acid levels as weighr\%, of total fatty acid levels in plasma phospholipids or milk total fat

b. $\quad A D=$ atcoic dermatitis at the age of 1 year according to UK.Wonking party probability score of atopic dermattis

c. SCORAD = severity of atcpic dermattis in intants at the age of 1 year

d. Based on student-1 test statistics, $95 \% \mathrm{CL}=95 \%$ confidence limits for difterence in metabolic index $n-6$ taty acids between high and low tertile 
Table 4 Perinatal n-3 metabolic index related to atopic outcomes in the intant at age 1 year

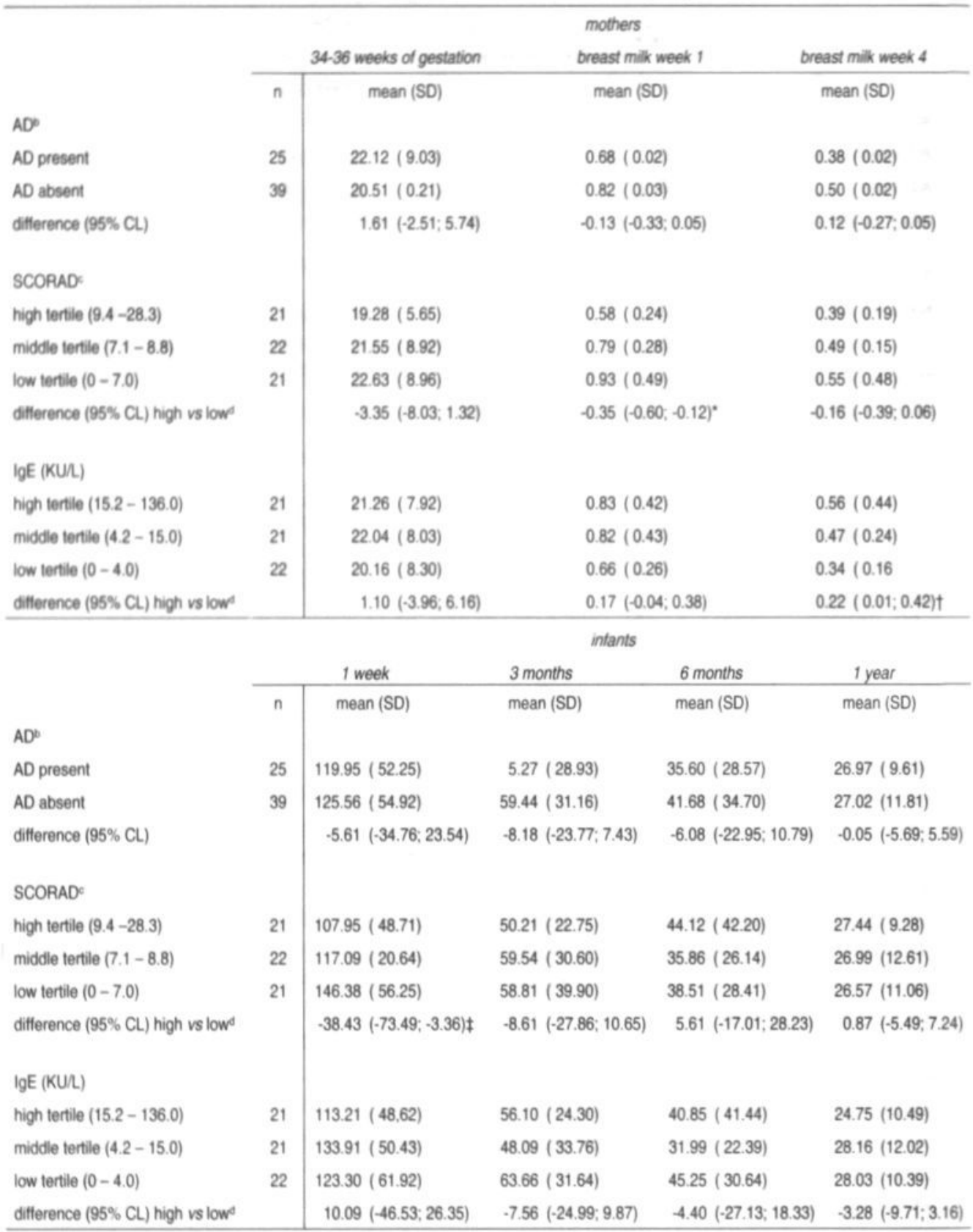

$\mathrm{n}=$ number of participants

a: $n-3$ metabolic index computed as: $[(18: 4 n-3+20: 5 n-3+22: 5 n-3+22: 6 n-3)(18: 3 n-3)) ;$ where $18: 4 n-3$ etc. are fatty acid levels as weight\% of total fatty acid levels in plasma phospholipids or milik total fat

b: $\quad A D=$ atopic dermatitis at the age of 1 year according to UK-Working party probability score of atopic dermatitis

c. $\quad S C O R A D=$ severity of atopic dermatitis in infants at the age of 1 year

d: Based on student- $t$ test statistics, $95 \% \mathrm{CL}=95 \%$ confidence limits for difference in metabolic index $n-3$ fatty acids between high and low tertile $\cdot P=0.005 ; P=0.03 ; \uparrow=0.04$ 
Table 5 Perinatal $n-6 / n-3$ ration related to atopic outcomes in the infant at age 1 year

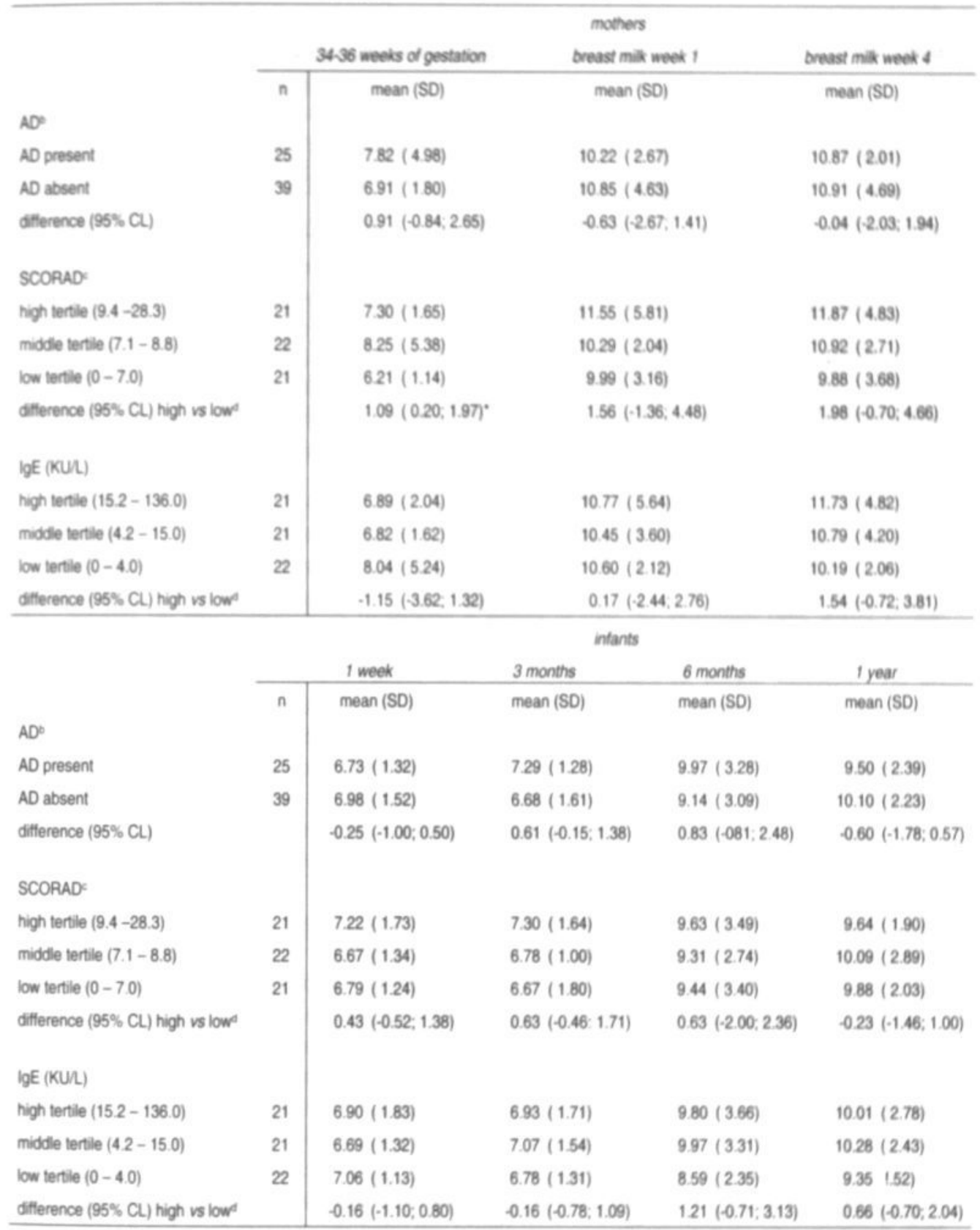

\footnotetext{
$n=$ number of participants
}

a. $n-6 / n-3$ ratio computed as: total $n-6$ EFAnotal $n-3$ EFA: where $n-6$ and $n 3$ EFA levels are fatty acid levels as weight\% of total fatty acid levels in plasma phospholipids or milk total tat

b. $\quad A D=$ atopic dermatitis at the age of 1 year according to UK-Working party probability score of atopic dermatifis

c. SCORAD $=$ severity of atopic dermatitis in intants at the age of 1 year

d. Based on studentt lest statistics, $95 \% Q=95 \%$ confidence interval for diflerence in $n-6 / n-3$ ratio between high and low tertile "P $=0.002$ 
Table 6 Perinatal DHA sufficiency indexa related to atopic outcomes in the infant at age 1 year

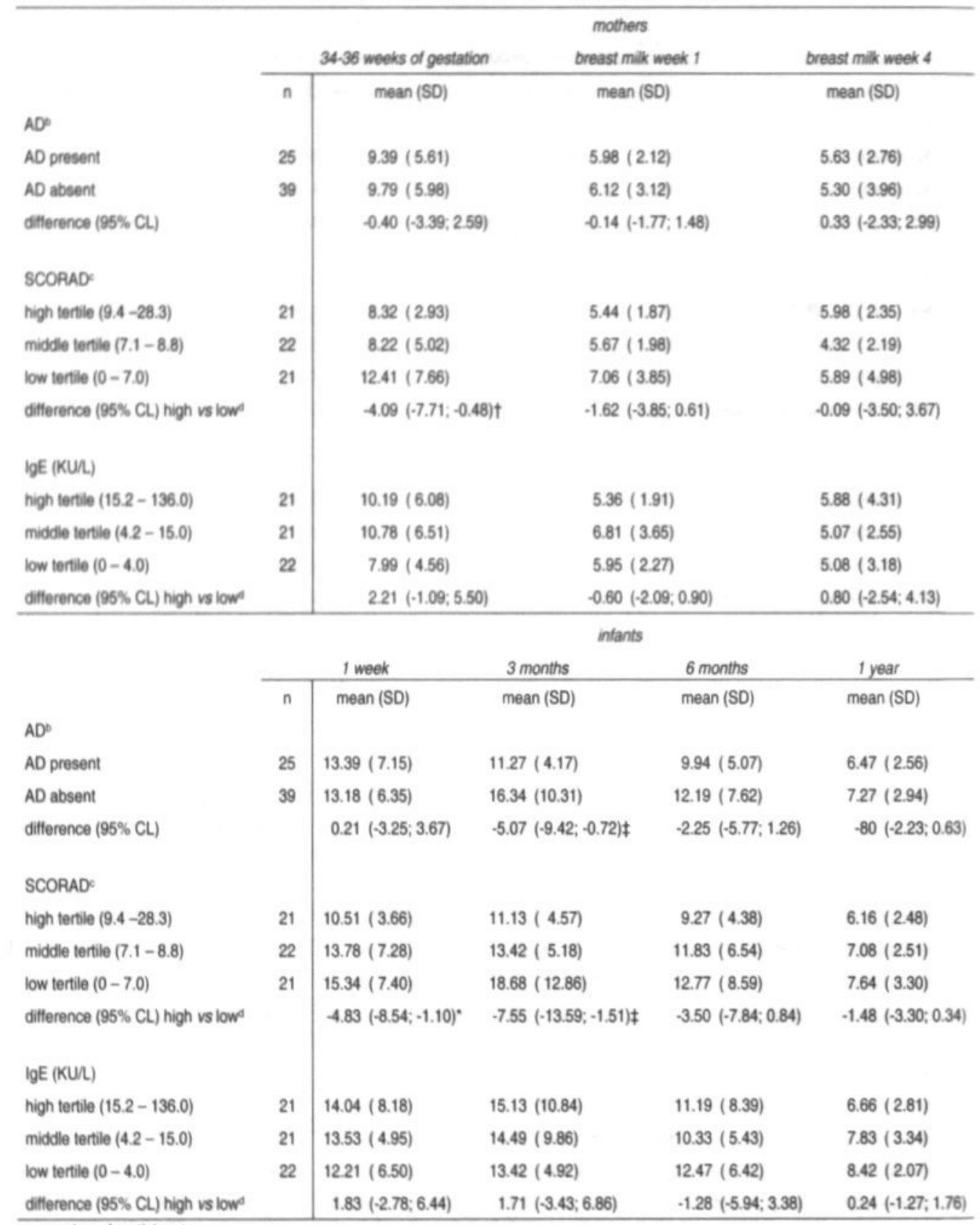

$\mathrm{n}=$ number of participants

a: DHA sufficiency index $=$ docosahexaenoic $(22: 6 n-3)$ sufficiency index computed as: $22: 6 n-3 / 22: 5 n-6$ : where $22: 6 n-3$ and $22: 5 n-6$ are fatty acid levels as weight\% of total fatty acid levels in plasma phospholipids or milk total fat

b: $\quad A D=$ atopic dermatitis at the age of 1 year according to UK-Working party probability score of atopic dermatitis

c: SCORAD $=$ severity of atopic dermatitis in intants at the age of 1 year

d. Based on student-1 test statistics, $95 \% \mathrm{CL}=95 \%$ confidence limits for difference in DHA sufficiency index between high and low tertile ${ }^{\circ} P=0.001, \neq P=0.02, \dagger P=0.03$ 


\section{DEFICIENCY MARKERS}

Levels of 20:3n-9, the deficiency marker of general n-6 and n-3 EFA status, did not differ between the groups of infants.

As outlined in the methods section, $22: 5 n-6$ is a marker for dietary deficiency of 22:6n-3. In infants with high SCORAD vs. infants with low SCORAD at the age of one week, three months and six months $22: 5 n-6$ was $32 \%, 38 \%$ and $30 \%$ higher, respectively $(P<0.02)$ (data not shown).

As shown in Table 6, the DHA sufficiency index at three months of age was $49 \%$ lower in plasma of infants with $A D$ compared to infants without $A D(P=0.02)$. Comparing infants with high vs. Iow SCORAD, DHA sufficiency index was lower in the mother during pregnancy (53\% lower; $P=0.03)$, and in the infant at one week and three months $(31 \%$ lower; $P=0.01$ and $58 \%$ lower, $P=0.02$, respectively). Although at other time points the DHA sufficiency index values tended to be lower in infants with high SCORAD compared to infants with low SCORAD, none of the differences reached statistical significance. No statistical significant differences between infants in high vs. low IgE were observed.

\section{DISCUSSION}

We focused on two hypotheses regarding a possible role for essential fatty acids in the aetiology of atopic dermatitis. Earlier observational studies suggested that an abnormal metabolism of n-6 EFAs is associated with the development of atopic disease. ${ }^{7}$ As an explanation, a reduced conversion of LA to its derivatives was suggested, possibly due to an impaired activity of D6D. ${ }^{3}$ Galli and co-workers ${ }^{8}$ were the first to demonstrate that changes in n-6 EFAs precede the development of atopic dermatitis. The concept of a causal role of low EFA supply in the aetiology of atopic dermatitis was also based on studies by Wright and Bolton" and by Businco et al. ${ }^{12}$ who reported lower levels of LA derivatives and lower DHA levels, but slightly higher levels of LA and ALA in breast milk from mothers whose infants developed atopic dermatitis.

In the present study, the metabolic indices of the n-6 and n-3 EFAs were utilised as an indicator of the D6D enzyme activity as Businco et al. ${ }^{12}$ did. The only consistent relation with atopy that we found was a lower n-3 metabolic index in breast milk of the mothers, and in infants' plasma at one week, in infants with the highest SCORAD at the age of one year.

An important difference between the $n-6$ metabolic index and the n-3 metabolic index is that the most abundant source of $n-6$ LCPs is the parent fatty acid, LA, so that n-6 metabolic index is not so much dependent on dietary supply as on the elongation and desaturation pathway. As a result, the n-6 metabolic index is a more accurate means to assess D6D activity than the n-3 metabolic index. Furthermore, LCPs of the n-3 EFA series can be derived from ALA, but the major source of n-3 LCPs is food. ${ }^{1}$ Based on the absence of a difference in n-6 metabolic index between atopic and non-atopic infants, we conclude that the development of atopy is not related to D6D activity. Since the exclusively breastfed infant is thought to completely depend on the EFA supply by the mother, the results rather mirror the mothers' EFA status than the D6D activity in the infant as such.

Our results do not confirm the differences observed by Galli in the individual n-6 EFAs between infants who developed AD and those who did not. Unfortunately, these authors did 
not report on n-3 EFAs. Also, the present study does not confirm the lower levels of n-6 LCPs in breast milk from mothers of atopic children as reported by Wright and Bolton " and by Businco, ${ }^{12}$ but it does confirm the lower levels of $n-3$ LCPs in these studies. Since the studies of Wright and Bolton and of Businco were performed in Italy and Zimbabwe it is possible that differences in the results between these and our studies are due to a higher intake of linoleic acid and a higher ratio in n-6/n-3 EFAs in the Netherlands. ${ }^{28}$

Regarding the hypothesis by Black and Sharpe ${ }^{14}$ we did not find differences in the $n-6 / n-3$ ratio between atopic and non-atopic infants or their mothers. However, we found that the DHA sufficiency index was lower at the age of three months in the infants with AD. It was also lower in the mothers and in the infants at the age of one week and three months for the infants in high vs. Iow SCORAD. In contrast to the DHA sufficiency index, DHA deficiency index was not different between groups, except for the one occasion when comparing the infants with high vs. Iow SCORAD at one week. This seemingly discrepancy between the two indices could be explained by the abundant supply of n-6 EFAs resulting in a large quantity of both $22: 4 n-6$ and $22: 5 n-6$. Therefore, the results point to a relative $n-3 E F A$ deficiency rather than a n-6 EFA overload. With respect to individual n-3 LCPs, whenever we found differences, they were always lower, in infants who were to develop AD (in breast milk at four weeks) or with the severest degree of atopic dermatitis (in utero and in breast milk). Results of the present study indicate that n-3 LCP status is related to the severity of atopic dermatitis and to a lesser degree to the development of $A D$, but not to the development of high $\lg E$ at the age of 1 year.

As an explanation for their hypothesis Black and Sharpe ${ }^{14}$ proposed that an increased intake of dietary n-6 EFAs and a decreased intake of n-3 EFAs influence the risk of atopic disease in two ways. Firstly, the higher $n-6 / n-3$ ratio increases the relative levels of $A A$ and hence increases the formation of prostaglandin E2. Secondly, high dietary n-3 EFA intake, especially of 20:5n-3 and DHA, competitively inhibits the action of cyclo-oxygenase, and thus lowers the formation of prostaglandin E2 from AA. A high prostaglandin E2 production specifically stimulates the formation of IL-4 and inhibits the formation of IFN-gamma, thus favouring a Th2-type skewed immunity. This mechanism pertains to the allergic (IgEmediated) component of atopy.

However, we found no relation between n-3 LCP status and $\lg E$ but only a relation with the severity of AD. N-3 EFAs have been reported to have anti-inflammatory properties in different conditions such as in rheumatoid arthritis. ${ }^{29.30}$ It has been suggested that in the skin epidermis 20:5n-3 and DHA have anti-inflammatory properties. ${ }^{32}$ Therefore, we suggest that $\mathrm{n}-3$ LCP status in the perinatal period does not so much influence the IgE-mediated component of atopic dermatitis but rather the inflammatory component.

In conclusion, results from this study are not consistent with the hypothesis that an inborn defect of the D6D in mothers or infants causes atopic disease. Our results do support a potential role in the etiology of AD of a relative deficiency of n-3 LCP intake by the mother, which determines n-3 status in the infants through intra-uterine supply and lactation. Major sources of dietary n-3 EFAs are green vegetables, lean meat and fatty fish. ${ }^{31}$ The intake of these n-3 EFAs has decreased in the last decades in West European countries. ${ }^{31}$ Hence, this could partly explain the rising trend in the incidence of atopic dermatitis and other atopic diseases. 


\section{ACKNOWLEDGEMENTS}

The authors would like to thank all midwives, parents and infants for participating. Alice Fleuren, Annemie Mordant, Diane Crook, Nancy Hendrix, Hasibe Aydeniz and Manon Meijs for their valuable and enthusiastic assistance. The study was supported by grants from Roche Vitamins Europe Ltd (Basel, Switzerland) and Friesland Coberco Dairy Foods (Leeuwarden, The Netherlands).

\section{REFERENCES}

1. Hornstra, G. (2000). "Essential fatty acids in mothers and their neonates." Am J Clin Nutr. 71(5 Suppl): 1262s-9s.

2. Brown, W. R. and A. E. Hansen (1937). "Arachidonic and Linolic Acid of the serum in nomal and eczematous human subjects." Proc Soc Exp Biol Med. 36: 113-117.

3. Manku, M. S., D. F. Horrobin, et al. (1982). "Reduced levels of prostaglandin precursors in the blood of atopic patients: defective delta-6-desaturase function as a biochemical basis for atopy." Prostaglandins Leukot Med. 9(6): 615-28.

4. Wright, S. and T. A. Sanders (1991). "Adipose tissue essential fatty acid composition in patients with atopic eczema." Eur J Clin Nutr. 45(10): 501-5.

5. Lindskov, R. and G. Holmer (1992). "Polyunsaturated fatty acids in plasma, red blood cells and mononuclear cell phospholipids of patients with atopic dermatitis." Allergy. 47(5): 517-21.

6. Thijs, C., A. Houwelingen, et al. (2000). "Essential fatty acids in breast milk of atopic mothers: comparison with non-atopic mothers, and effect of borage oil supplementation." Eur J Clin Nutr. 54(3): 234-8.

7. Horrobin, D. F. (1992). "Nutritional and medical importance of gamma-linolenic acid." Prog Lipid Res. 31(2): 163-94.

8. Galli, E., M. Picardo, et al. (1994). "Analysis of polyunsaturated fatty acids in newborn sera: a screening tool for atopic disease?" Br J Dermatol. 130(6): 752-6.

9. Saarinen, U. M. and M. Kajosaari (1995). "Breastfeeding as prophylaxis against atopic disease: prospective follow-up study until 17 years old [see comments]." Lancet. 346(8982): 1065-9.

10. Hanson, L. A. (1998). "Breastfeeding provides passive and likely long-lasting active immunity." Ann Allergy. 81(6): 523-33.

11. Wright, S. and C. Bolton (1989). "Breast milk fatty acids in mothers of children with atopic eczema." Br J Nutr. 62(3): 693-7.

12. Businco, L., M. loppi, et al. (1993). "Breast milk from mothers of children with newly developed atopic eczema has low levels of long chain polyunsaturated fatty acids." J Allergy Clin Immunol. 91(6): 1134-9.

13. Melnik, B. C. (1990). "A chance for the prevention of atopic diseases]." Monatsschr Kinderheilkd. 138(3): $162-6$

14. Black, P. N. and S. Sharpe (1997). "Dietary fat and asthma: is there a connection?" Eur Respir J. 10(1): 6-12.

15. Kankaanpaa, P., Y. Sutas, et al. (1999). "Dietary fatty acids and allergy." Ann Med. 31(4): 282-7.

16. van Gool, C. J. A. W., A. Houwelingen v., et al. (2000). The essential fatty acid status in phenylketonuria patients under treatment." J Nutr Biochem. 11(11): 543-547.

17. Otto, S. J., M. M. Foreman van Drongelen, et al. (1997). "Effects of storage on venous and capillary blood samples: the influence of deferoxamine and butylated hydroxytoluene on the fatty acid alterations in red blood cell phospholipids." Eur J Clin Chem Clin Biochem. 35(12): 907-13.

18. Kaluzny, M. A., L. A. Duncan, et al. (1985). "Rapid separation of lipid classes in high yield and purity using bonded phase columns." J Lipid Res. 26(1): 135-40.

19. Al, M. D., A. C. van Houwelingen, et al. (1995). Maternal essential fatty acid patterns during normal pregnancy and their relationship to the neonatal essential fatty acid status." Br J Nutr. 74(1): 55-68.

20. Neuringer, M., W. E. Connor, et al. (1986). "Biochemical and functional effects of prenatal and postnatal omega 3 fatty acid deficiency on retina and brain in rhesus monkeys." Proceedings of the National Academy of Sciences of the United States of America 83(11): 4021-5. 
21. Hoffman, D. R. and R. Uauy (1992). "Essentiality of dietary omega 3 fatty acids for premature infants: plasma and red blood cell fatty acid composition." Lipids. 27(11): 886-95.

22. Paganelli, R., I. J. Ansotegui, et al. (1998). "Specific IgE antibodies in the diagnosis of atopic disease. Clinical evaluation of a new in vitro test system, UniCAP, in six European allergy clinics." Allergy. 53(8): 763-8.

23. Aalberse, R. C., H. G. Brummelhuis, et al. (1973). "The purification of human polyclonal IgE by immunosorption." Immunochemistry. 10(5): 295-303.

24. Williams, H. C., P. G. Burney, et al. (1994). "The U.K. Working Party's Diagnostic Criteria for Atopic Dermatitis. III. Independent hospital validation." Br J Dermatol. 131(3): 406-16.

25. Williams, H. C., P. G. Burney, et al. (1994). "The U.K. Working Party's Diagnostic Criteria for Atopic Dermatitis. II. Observer variation of clinical diagnosis and signs of atopic dermatitis." $\mathrm{Br} \mathrm{J}$ Dermatol. 131(3): 397-405.

26. European Task Force on Atopic Dermatitis (1993). "Severity scoring of atopic dermatitis: the SCORAD index. Consensus Report of the European Task Force on Atopic Dermatitis." Dermatology. 186(1): 23-31.

27. Kunz, B., A. P. Oranje, et al. (1997). "Clinical validation and guidelines for the SCORAD index: consensus report of the European Task Force on Atopic Dermatitis." Dermatology. 195(1)): 10-9.

28. Hulshof, K. F., M. A. van Erp Baart, et al. (1999). "Intake of fatty acids in western Europe with emphasis on trans fatty acids: the TRANSFAIR Study." Eur J Clin Nutr. 53(2): 143-57.

29. James, M. J., R. A. Gibson, et al. (2000). "Dietary polyunsaturated fatty acids and inflammatory mediator production." Am J Clin Nutr. 71 (1 Suppl): 343s-8s.

30. Kremer, J. M. (2000). "n-3 fatty acid supplements in meumatoid arthritis." Am J Clin Nutr. 71(1 Suppl): $349 \mathrm{~s}-51 \mathrm{~s}$.

31. Simopoulos, A. P. (1999). "Essential fatty acids in health and chronic disease." Am J Clin Nutr. The $70(3$ Suppl): 560 s-569s.

32. Ziboh, V. A., C. C. Miller, et al. (2000). "Metabolism of polyunsaturated fatty acids by skin epidermal enzymes: generation of antiinflammatory and antiproliferative metabolites." Am J Clin Nutr. 71(1 Suppl): $361 \mathrm{~s}-6 \mathrm{~s}$. 


\section{Gamma-linolenic acid supplementation to prevent atopy - a randomized controlled trial in infants at high familial risk (the EFAtop study)}

Christel J.A.W. van Gool'

Carel Thijs'

Charles J.M. Henquet ${ }^{2}$

Adriana C. v. Houwelingen'

Pieter C. Dagnelie'

Jaap Schrander ${ }^{4}$

Paul P.C.A. Menheere

Piet A. van den Brandt'

'Department of Epidemiology. Maastricht University

2Department of Dermatology, Mastricht University

'Department of Human Biology, Mastricht University

'Department of Pediatrics, Mastricht University

sDepartment of Clinical Chemistry, University Hospital Mastricht 


\section{ABSTRACT}

\section{BACKGROUND}

Studies suggest that low levels of $n-6$ long chain polyenes in early life are correlated to atopic disease in later life.

\section{OBJECTTVE}

To investigate the possible preventive effect of gamma-linolenic acid (GLA) supplementation on the development of atopic dermatitis in infants at risk.

\section{DESIGN}

In a double blind, randomized, placebo-controlled trial, formula-fed infants $(n=118)$ with a maternal history of atopic disease were supplemented daily with borage oil supplement (containing $100 \mathrm{mg}$ of GLA) for the first six months of life, or sunflower oil supplement as a placebo. Main outcome measures were: incidence of atopic dermatitis (AD) in the first year of life (UK Working Party criteria), severity of atopic dermatitis (SCORAD), and serum total $\lg \mathrm{E}$ at the age of one year.

\section{RESULTS}

An intention-to-treat analysis showed a non-significant $(P>0.05)$ favorable trend for the clinical atopic outcomes (AD, SCORAD) in the GLA group compared to the placebo group. The increase in GLA levels in plasma phospholipids between baseline and three months was strongly negatively associated with severity of atopic dermatitis at one year (high vs. low tertile of SCORAD: odds ratio $=0.17$ (95\% confidence interval 0.05 $-0.60), P=0.01$ for large increase in GLA ( $83^{\text {rd }}$ percentile) vs. small increase in GLA $\left(17^{\text {th }}\right.$ percentile)). No effect was present on total serum $\lg \mathrm{E}$.

\section{CONCLUSION}

Early supplementation of GLA in children at high familial risk does not prevent the expression of atopy as reflected by total serum $\mathrm{IgE}$, but alleviates the severity of atopic dermatitis in later infancy in these children. 


\section{INTRODUCTION}

Essential fatty acids (EFAs) are believed to be involved in the etiology of atopic disease.' In the n-6 EFA series, linoleic acid (LA, 18:2n-6), derived from food, is subsequently converted into gamma-linolenic acid (GLA, 18:3n-6) and longer-chain polyenes (LCPs) such as dihomogamma-linolenic acid (DGLA, 20:3n-6) and arachidonic acid (AA, 20:4n-6). Although LCPs of the n-3 EFA series can be derived from alpha-linolenic acid (ALA, 18:3n-3), the major source of $n-3$ LCPs is food. As early as 1937, lower levels of AA were reported in the serum of children with atopic dermatitis. ${ }^{2}$ More recent studies have shown higher levels of LA and substantially lower levels of its LCPs in the blood of these patients. ${ }^{3-5}$ In newborn infants with a family history of atopic disease, lower n-6 LCP levels in umbilical cord blood were found to precede the development of atopic dermatitis. ${ }^{6}$ One suggested explanation for these findings was a reduced conversion of LA into GLA and subsequent LCPs, possibly caused by impaired activity of the enzyme delta-6-desaturase (D6D). ${ }^{3.7}$ Other studies showed that breast milk from mothers whose infants subsequently developed atopic dermatitis (AD) contained less n-6 LCPs than milk from mothers whose infants remained unaffected. ${ }^{\text {. }}$ Unlike breast milk, infant formulas until recently contained only linoleic acid and alphalinolenic acid as EFAs. Only lately are some brands of formula being enriched with LCPs, some including GLA.

Intervention studies with GLA supplementation in patients with AD have shown inconsistent results. Most trials were carried out in a mixed population of adults and children; only two trials were restricted to children. ${ }^{10,11}$ All of these trials aimed at decreasing the severity of existing eczema; no preventive trials have so far been conducted.

A possible role of GLA in the prevention of atopy in early life has been postulated by Melnik, ${ }^{12}$ based on the following observations: 1) body composition with respect to EFAs in newborn infants is entirely dependent on intra-uterine supply and the subsequent choice of breast- vs. bottle-feeding: ${ }^{13}$ 2) mothers of atopic infants have lower n-6 LCP levels in their breast milk than mothers of non-atopic infants ${ }^{9}$ and 3 ) infants who show atopic symptoms at the age of one year have on average consistently and significantly lower concentrations of $n$ 6 LCPs in umbilical cord blood and in serum at one and three months of age, compared to infants who remain unaffected. ${ }^{6}$ Prostaglandins derived from n-6 LCPs are thought to play a role in the maturation of the immune system. ${ }^{12}$ Since the conversion of LA to GLA is thought to be the rate-limiting step in the total chain of conversions, ${ }^{14}$ supplementation of GLA in infancy might compensate the lower n-6 LCP levels and prevent atopy or decrease its severity in infants, especially if the mother has an atopic constitution.

The present study aimed at investigating whether GLA supplementation protects against the development of atopy in high-risk, formula-fed infants.

\section{MATERIALS AND METHODS}

\section{STUDY DESIGN AND POPULATION}

The present study was a double blind, randomized, placebo-controlled trial in infants at high risk of atopic dermatitis. Subjects eligible for the study were atopic pregnant women and their infants. They were recruited between October 1997 and April 2000 by midwives and via 
advertisements in local newspapers in the provinces of Limburg and Noord-Brabant, the Netherlands. The atopic status of both parents was assessed using a validated telephone questionnaire. Inclusion criteria for atopic mothers were: a history of allergic asthma or allergic rhinoconjunctivitis with relation to aeroallergen exposure, or of atopic dermatitis, or a history of positive allergen test, or improvement of asthma or rhino conjunctivitis complaints upon using antihistamine drugs or anti-asthma drugs. Exclusion criteria were: diabetes treated with medication and/or diet, (pre-)eclampsia and metabolic diseases.

Infants were born between December 1997 and May 2000. Inclusion criteria for the infants were: gestational age of at least 38 weeks, birth weight $>2500$ grams, an uncomplicated perinatal period, and being exclusively formula-fed from two weeks of age. All data were collected at the participants' homes except for the final visit at the age of one year, which took place at the University Hospital Maastricht, the Netherlands. The study was approved by the medical ethics committee of the University Hospital Maastricht and written informed consent was obtained from both parents.

\section{RANDOMIZATION, INTERVENTION AND COMPLIANCE}

After inclusion, infants were prestratified according to atopic status of the father (as the main prognostic factor additional to the mothers atopy) and then randomly assigned to the experimental and placebo groups, using block randomization in blocks of four. Intervention started as soon as possible after baseline blood sampling at the age of $7( \pm 2)$ days, or 14 days at the latest. The supplementation period ended when the infant reached the age of six months. The intervention comprised a daily supplement that was given as 1 gram of powder, consisting of fish gelatin (135 mg), maltodextrin (397 mg), silicic acid (21 mg) and oil (446 $\mathrm{mg}$ ). The oil was either borage oil (Borago Officinalis, verum); or sunflower oil (placebo), which closely resembled borage oil with respect to fatty acid composition, except that the GLA in verum was replaced by oleic acid (18:1n-9) (Table 1). Both supplements contained vitamins $\mathrm{C}$ and $\mathrm{E}$ as antioxidants, and were provided by $\mathrm{F}$. Hoffmann-LaRoche, Basel, Switzerland. The powder was packaged in low-oxygen sachets in order to blind the investigators and parents for possible differences in smell and appearance of the powder. In order to have the supplement distributed evenly over the day, parents were instructed to put the supplement in the total amount of milk made for one day. The dose was chosen to reflect upper normal levels of GLA in human milk. Mean GLA levels reported for human milk (as weight percentages of total milk lipids) in Western populations vary from $0.07 w t{ }^{9}$ to $0.35 w t \% .{ }^{15}$ Other studies reviewed by Jensen (1992) showed levels in between these values. ${ }^{13}$ We aimed at the highest level, namely 0.35 wt\%, of GLA in human milk. At a total typical daily output of about $750 \mathrm{~g}$ of milk with $4 \mathrm{wt} \%$ total fat, this amounts to approximately $100 \mathrm{mg}$ GLA per day. The actual amount of GLA present in the GLA supplement was $23.1 \%$ of the $446 \mathrm{mg}$ of borage oil in a daily dose, i.e. $103 \mathrm{mg}$ GLA per day.

Compliance was measured by counting the number of sachets returned. Mothers were instructed to keep a diary focusing on events that disturbed the infants' food or supplement intake. Compliance was considered to be complete if at least $85 \%$ of the sachets had been used during the supplementation period as a whole. We expected that the rise in plasma GLA would reflect compliance on the basis of earlier supplementation studies ${ }^{13}$ and therefore measured the rise in plasma GLA from baseline (before supplementation) up to three and six months. 
Table 1 Fatty acid profles of verum (borage oll) and placebo supplements (\% of total tatty acids)

\begin{tabular}{lrc}
\hline & Verum (borage oil) & Placebo \\
\hline SAFA & & \\
$14: 0$ & $0.1 \%$ & $0.1 \%$ \\
$16: 0$ & $10.7 \%$ & $5.8 \%$ \\
$18: 0$ & $3.8 \%$ & $3.9 \%$ \\
20.0 & $0.2 \%$ & $0.3 \%$ \\
22.0 & $0.2 \%$ & $0.7 \%$ \\
Total SAFA & $15.0 \%$ & $10.8 \%$ \\
MUFA & & \\
$18: 1 n-9$ & $15.4 \%$ & $50.7 \%$ \\
$20: 1 n-9$ & $4.0 \%$ & $0.3 \%$ \\
$22: 1 n-9$ & $2.7 \%$ & 0.0 \\
Total MUFA & $22.1 \%$ & $51.0 \%$ \\
PUFA & & \\
$18: 2$ n-6, LA & $36.8 \%$ & $36.6 \%$ \\
$18: 3$ n-6, GLA & $23.1 \%$ & $0.0 \%$ \\
$18: 3$ n-3, ALA & $0.2 \%$ & $0.2 \%$ \\
Total PUFA & $60.1 \%$ & $36.8 \%$ \\
Other/unknown & $2.8 \%$ & $1.4 \%$ \\
Total & $100 \%$ & $100 \%$ \\
\hline
\end{tabular}

\section{SAMPLING AND LABORATORY ANALYSES}

Blood was collected from the mothers by venepuncture of the median cubital vein at 34-36 weeks of gestation, and from the infants at the ages of one week, three months and six months by heel prick or finger prick and at the age of one year by venepuncture of the hand vein or of the median cubital vein. Blood was collected in EDTA-containing tubes (Becton Dickinson, NJ USA) and serum separator tubes (Sherwood-Davis \& Geck, StLouis, USA). The samples were transported on ice/water mixture and, within 24 hours after collection, centrifuged for 10 minutes $\left(3000 \mathrm{~g}\right.$ for 10 minutes at $4^{\circ} \mathrm{C}$ ), and stored at $-20^{\circ} \mathrm{C}$ (serum) or $50^{\circ} \mathrm{C}$ (plasma) under nitrogen until analysis. If an insufficient amount of venous blood was acquired, finger prick blood was collected on blotting paper for total serum IgE analysis.

Fatty acids (FAs) were analyzed as previously described. ${ }^{16}$ Lipid extracts were prepared from plasma samples, and phospholipid (PL) fractions separated using aminopropyl-bonded phase columns. ${ }^{17}$ PLs were hydrolyzed and FAs transmethylated with boron trifluoride (SIGMA) in methanol. The composition of the FA methyl esters obtained was determined by capillary gas chromatography using a polar capillary column (CPSil 88; Chrompack, Middelburg, the Netherlands), with helium as the carrier gas. The amount of each FA was quantified by adding an internal standard (dinonadecanoyl [19:0]-phosphatidylcholine, SIGMA). Results are reported as weight percentages (wt\%) of total FAs, computed as previously described. ${ }^{18}$

Screening tests for total IgE and common aeroallergens and food allergens (Phadiatop and Fx5, respectively) were performed in serum from infants at one year of age using the Pharmacia Unicap system (Pharmacia Upjohn, Upsala, Sweden) as described elsewhere. ${ }^{19}$ 
Measurement of total IgE in eluded blood spot material was performed in a sandwich assay, as previously described, ${ }^{20}$ with minor modifications: a mixture of anti-human IgE monoclonal antibodies was coupled to Sepharose $4 \mathrm{~B}$ to catch $\mathrm{IgE}$; Sepharose-bound IgE was detected using radio-labeled antibodies against human $\mathrm{lg} E$, raised in sheep.

\section{CLINICAL OUTCOME PARAMETERS AND ADVERSE EVENTS}

At the follow-up hospital visit at the age of one year, a trained dermatologist $(\mathrm{CH})$ determined the clinical diagnosis of atopic dermatitis using the UK Working Party criteria. ${ }^{21,22}$ Briefly, the probability of the presence of atopic dermatitis was derived from the presence of four clinical symptoms: (1) presence of itchy rash (PIR, coded as 1=present, $0=$ absent), (2) history of or (3) visible flexural dermatitis (HFD or VFD, $1=$ present, $0=a b s e n t$ ), and (4) onset before 2 years of age (OB2, 1 =present, $0=a b s e n t)$. HFD and VFD were modified to the extensor side of the limbs to match the typical clinical predilection sites in infants. ${ }^{21.22}$ The UK-Working Party Probability Score of atopic dermatitis (AD) was then computed as: probability $(A D)=$ odds $(A D) /$ odds $(A D)+1]$; where odds $(A D)=\exp [(4.86+1.84(H F D)+$ $3.46(\mathrm{OB} 2)+2.09$ (VFD) +1.71 (PIR).$^{21.22}$ In the present study, infants with a probability of $A D$ $>0.50$ were considered to have $A D$.

Severity of dermatitis was scored by one dermatologist $(\mathrm{CH})$ using the SCORAD clinical criteria $(23,24)$. These criteria include measuring the size of the affected area (A) and scoring the intensity of the dermatitis (B), with five criteria for scoring intensity: erythema, edema/papulation, oozing/crusting, excoriation and lichenification (each with scores 0-2). The SCORAD index was computed as: $\mathrm{A} / 5+7 \mathrm{~B} / 2$. $^{23,24}$

At the same time, the SCORAD was scored independently by one of the other trained investigators (CvG, CT) to assess interobserver variability with the dermatologist. Parents were asked to report on severity of itch and sleeplessness as a result of itch, using a visual analogue scale ranging from 0 (smiling baby) to 100 (crying baby). Parents were also asked to bring the infants' medication to the hospital, and use of emollients and corticosteroids was carefully noted.

\section{SIZE OF THE STUDY POPULATION}

The incidence of atopic dermatitis in the first year of life reported in the literature ranges from $22-33 \%{ }^{25}$ to $38 \%{ }^{26}$ (average $35.5 \%$ ) in breast-fed infants at risk; in formula-fed infants, the incidence of atopic dermatitis ranged from $49 \%{ }^{25}$ to $57 \%{ }^{27}$ (average $53 \%$ ). Several studies have reported a stronger maternal than paternal inheritance; the only study to report incidences separately for atopic mothers and atopic fathers found an incidence of atopic eczema of $47 \%$ in children with atopic mothers and of $10 \%$ in children with atopic fathers. ${ }^{28}$ Assuming an average incidence of $53 \%$ in the placebo group, we aimed at a $41 \%$ risk reduction in the intervention group (equaling an incidence of about $31 \%$, which is equivalent to the incidence of atopic dermatitis in breast-fed infants). For a power of $80 \%$ (at alpha $=$ 0.05 ; one-sided) the study size needed to detect this risk reduction was calculated to be 122 infants. 


\section{STATISTICAL ANALYSIS}

Statistical analyses were performed with the SPSS for Windows program (version 10.0, SPSS, Inc., Chicago, IL, USA). AD was the main outcome variable, with SCORAD and total and specific $\lg E$ as secondary outcome measures. Total serum IgE was not normally distributed and was therefore transformed to its natural logarithm (log $\lg E)$. Spearman's rank correlations were calculated to assess the correlation between the different outcome parameters (SCORAD, log IgE, UK-Working Party Probability Score).

In the intention-to-treat analysis comparing GLA and placebo groups for binary outcome measures (AD, reported itch, topical steroid use, emollient use, and positive Fx5), odds ratios were computed using logistic regression analysis. Differences in mean SCORAD and $\log \lg E$ between the GLA and placebo groups were tested for statistical significance using Student ttest (alpha $=0.05$, two-sided); $95 \%$ Confidence intervals $(\mathrm{CI})$ are reported throughout.

Logistic regression analysis was performed to test the association between the rise of serum GLA between baseline (age one week) and three months of age and between baseline and six months of age on the one hand, and $A D$ (present vs. absent) on the other. Since SCORAD was not distributed normally and log transformation did not overcome this problem, logistic regression analysis was also used to test the association between the higher vs. lower tertile of the SCORAD and the rise in GLA. Linear regression analysis was used to investigate the relationship between $\log \lg E$ at the age of one year and the rise in GLA; in addition, we performed logistic regression analysis with the higher vs. lower tertile of $\operatorname{lgE}$ as the outcome.

Potential confounders included as covariates in the logistic regression models were variables that are known to be associated with atopy: atopic status of the father, smoking during pregnancy, smoking by parents in the first year of life, age of the mother at delivery, IgE of the infant at one week, $\operatorname{lgE}$ of the mother, number of siblings, day-care attendance, educational level of the father, educational level of the mother, presence of a dog in the household, presence of a cat, and use of allergen-free bed covers.

\section{RESULTS}

One hundred and twenty-one infants were included in the study. At the age of one year, 118 infants had completed follow-up, 58 in the GLA group and 60 in the placebo group (Fig. 1). The remaining three infants did not complete the supplementation and were also lost to follow-up.

Complete compliance (> $85 \%$ of the sachets used) was observed in $85 \%$ of the infants (51 in the GLA group and 51 in the placebo group). Five infants in each group had used between $50 \%$ and $85 \%$ of the sachets (semi-compliers), whereas four infants in the GLA group and two infants in the placebo group had used less than $50 \%$ (non-compliers) (Fig.1).

Adverse events were equally divided over the GLA and placebo groups in the initial 121 infants, with abdominal cramps (three infants) and bringing up milk (five infants) being the most frequent symptoms (Fig. 1). Both symptoms were usually temporary, but in most cases resulted in the parents withholding the supplements from the infant for a while (varying from two days to two weeks), to ensure that the symptoms were not caused by the supplements. 
Figure 1 Trial profile: follow-up, compliance and reported adverse events in the EFAtop study.

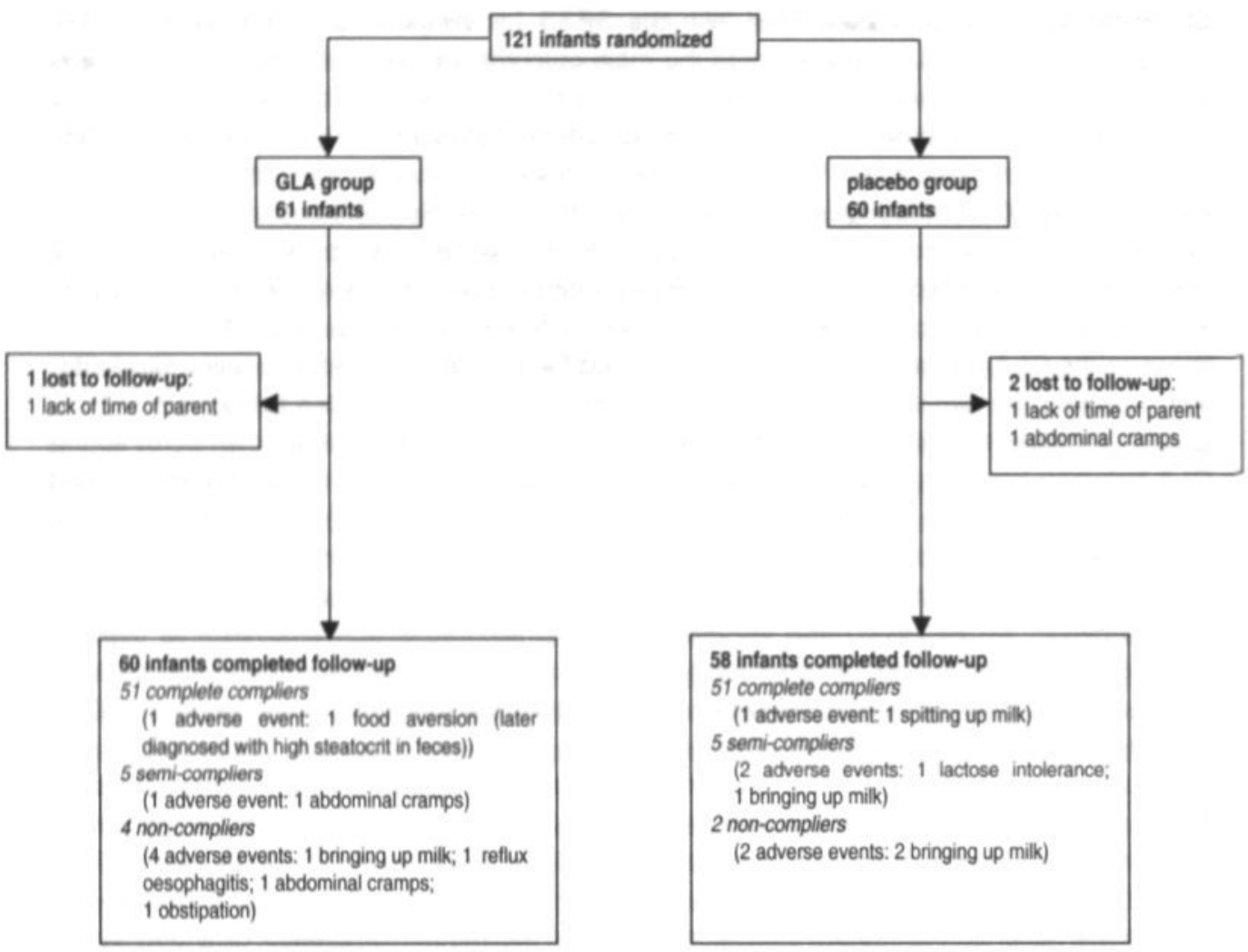

When the symptoms subsided, parents were asked to re-introduce the supplement slowly and to report whether the problems either recurred or stayed away.

The GLA and placebo groups were comparable with regard to baseline variables except for slighter higher, but not significantly, baseline levels of $\lg \mathrm{E}$ at one week of age, more presence of a carpet in the infants' bedroom and the lesser use of allergen-free bed covers in the GLA group (Table 2). In our population the LANALA ratio in infant formulas laid between 10:1 and 6:1 and the formulas contained no LCPs. Figure 2 shows the course of GLA, DGLA and AA levels in the infants' plasma phospholipids for the GLA and placebo groups. In the GLA group, compliers had distinctively higher GLA, DGLA and AA levels at three and six months compared to the placebo group, whereas semi- and non-compliers in the GLA group had levels close to the placebo group (Fig. 2). At three months, the levels of GLA, DGLA and AA in the total GLA group compared to the placebo group were, respectively, $70 \%, 24 \%$ and $13 \%$ higher. At six months, the levels of GLA, DGLA and AA were, respectively, $47 \%, 19 \%$ and $15 \%$ higher. When corrected for baseline levels (at one week), all these differences remained significantly different. 
Figure 2. GLA DGLA and AA levels in infants' plasma phospholipids in GLA and placebo groups (mean \pm SEM)
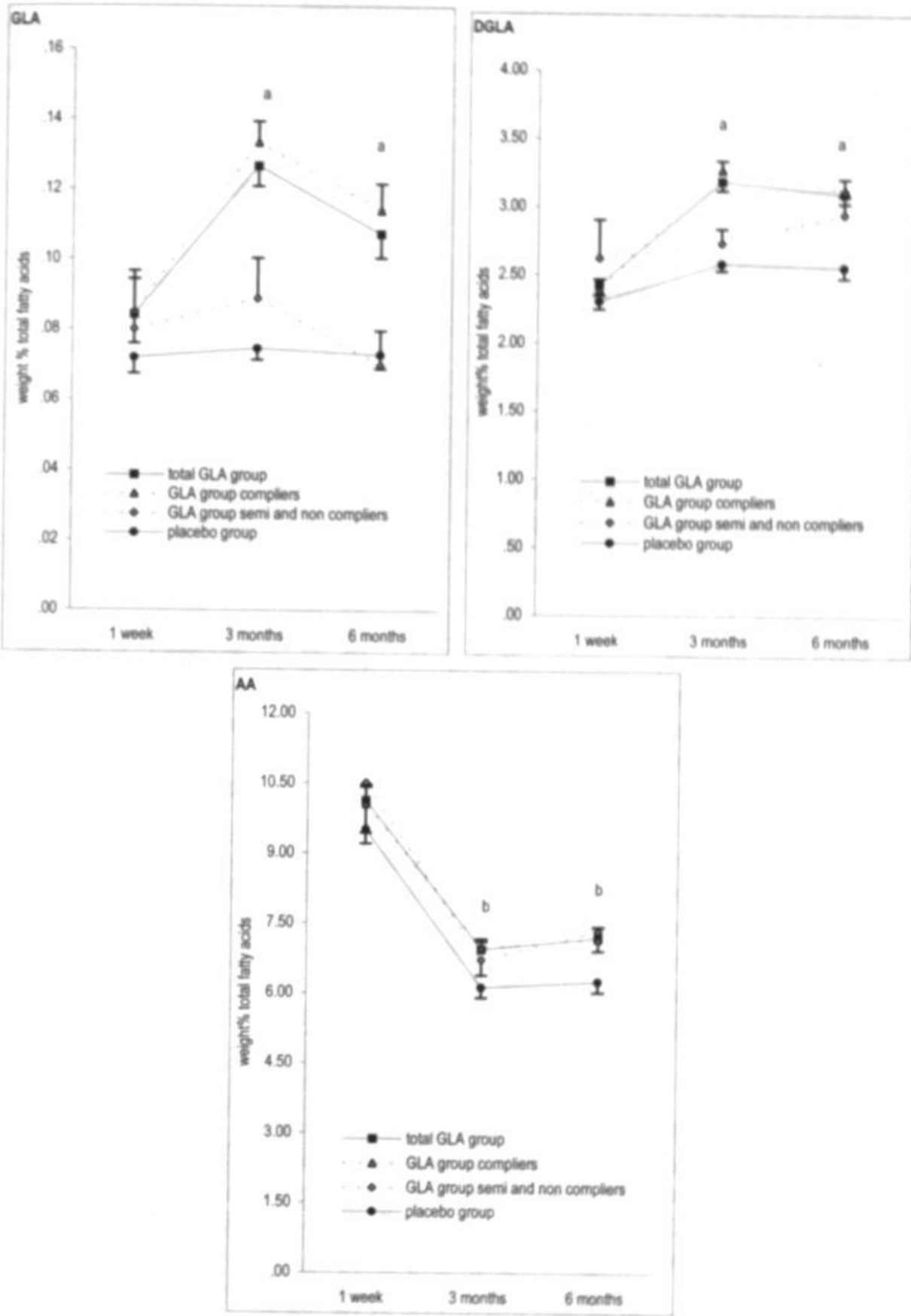

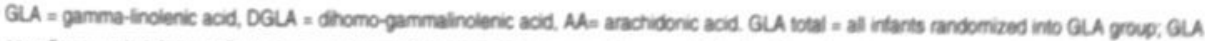
complens = only intants in GLA complant group: GLA semi and non-complens = only intarts of sem and non-complens in GUA group ne118 (n-60 QLA

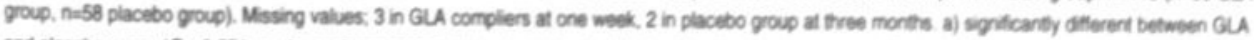

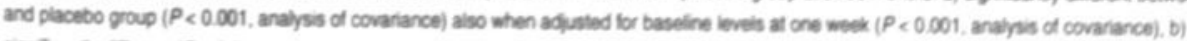

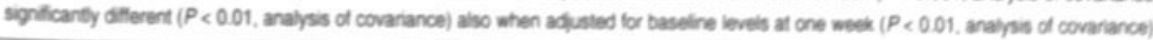


Table 2. Baseline variables and co-interventions between GLA and placebo groups

\begin{tabular}{|c|c|c|}
\hline & GLA & placebo \\
\hline Number of infants & 60 & 58 \\
\hline \multicolumn{3}{|l|}{ Family history } \\
\hline \multicolumn{3}{|l|}{ Atopic history of mother } \\
\hline asthma $(n)$ & 27 & 26 \\
\hline minitis (n) & 51 & 43 \\
\hline atopic eczema (n) & 23 & 20 \\
\hline positive allergen test in history $(n)$ & 47 & 41 \\
\hline use of antihistamine drugs in history ( $\mathrm{n}$ ) & 23 & 20 \\
\hline \multicolumn{3}{|l|}{ Total serum IgE of mother ( $\mathrm{n}$ in tertiles) *: } \\
\hline 1 - $52 \mathrm{KU} / \mathrm{L}, 53$ - $209 \mathrm{KU} / \mathrm{L}, 210$ - $5506 \mathrm{KU} / \mathrm{L}$ & $21,22,17$ & $18,17,23$ \\
\hline Positive aeroallergen screening test (Phadiatop) mother ( $n$ ) & 44 & 46 \\
\hline Positive food allergen screening test (Fx5) mother ( $n$ ) & 9 & 9 \\
\hline Atopic history of father $n$ (positive, uncertain/negative) & 22,38 & 21,37 \\
\hline Age of mother at time of delivery in years, mean (SD) & $31.2(4.0)$ & $30.5(3.9)$ \\
\hline Duration of pregnancy in weeks, mean (SD) & $40.1(1.3)$ & $40.1(1.1)$ \\
\hline \multicolumn{3}{|l|}{ Child } \\
\hline Girls $(\%)$ & $55 \%$ & $53 \%$ \\
\hline Birth weight in grams, mean (SD) & $3513(483)$ & $3528(463)$ \\
\hline Birth length in centimeters, mean (SD) & $53.8(13.4)$ & $53.2(11.3)$ \\
\hline Head circumference at birth in centimeters, mean (SD) & $34.9(1.6)$ & $34.8(1.4)$ \\
\hline \multicolumn{3}{|l|}{ Total serum lgE infants at age 1 week ( $\mathrm{n}$ in tertiles) ${ }^{\mathrm{a}}$ : } \\
\hline $0.00-0.10 \mathrm{KU} / \mathrm{L}, 0.11-0.73 \mathrm{KU} / \mathrm{L}, 0.74-21.00 \mathrm{KU} / \mathrm{L}$ & $23,19,16$ & $16,20,21$ \\
\hline \multicolumn{3}{|l|}{ Risk factors } \\
\hline Highest educational level either parent, ( $n$ ) (low, middle, high) & $4,34,22$ & $8,30,20$ \\
\hline Number of siblings $(n)(0,1,2$ or more $)$ & $27,22,11$ & $30,19,9$ \\
\hline Day-care attendance in days per week, mean (SD) & $0.92(1.06)$ & $1.04(1.13)$ \\
\hline Pets present in the house (n) (cat, dog, rabbit) & $5,19,2$ & $2,17,2$ \\
\hline Carpet in living room, in infant's bedroom, allergen-free cover infant's bed ( $n$ ) & $6,14,2$ & $5,3,6$ \\
\hline
\end{tabular}

-Tertiles based on total group (GLA and placebo), one missing in placebo group

We dichotomized the UK Working party probability score into AD absent (67 infants with a UK Working party probability score of 0.29 ) and AD present (51 infants with probabilities ranging from 0.69 to 0.95 ). The Spearman's rank correlation between the UK-Working Party probability score and SCORAD was $0.56(P=0.01)$. There was no correlation between the UK-Working Party probability score and $\log$ total serum $\lg E(r=0.12, P=0.21)$ or between SCORAD and $\log$ total serum $\lg E(r=0.09, P=0.29)$. In the intention-to-treat analyses, slightly fewer infants in the GLA group had developed AD at the age of one year compared to the placebo group (Table 3). 


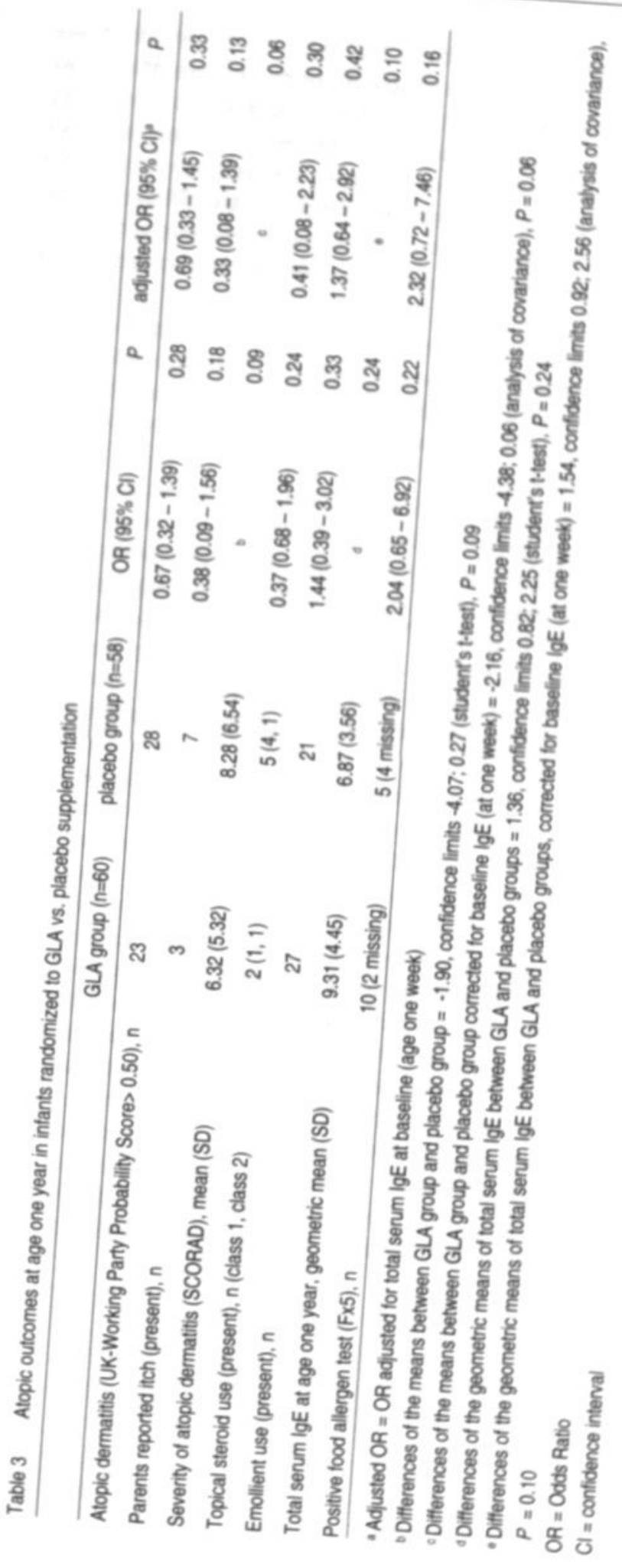


Infants in the GLA group showed a tendency toward lower SCORAD values, and for fewer parents to report itch and use of topical corticosteroids (Table 3). None of these differences reached statistical significance $(P>0.06)$. By contrast, the GLA group showed a tendency toward higher serum IgE values, as well as a non-significant tendency towards a higher number of positive food allergen tests (Fx5) at the age of one year, compared to the placebo group. When the analyses were restricted to the complete compliers, the differences for SCORAD and IgE remained roughly the same (Table 4). None of the differences in baseline $\operatorname{lgE}$ were statistical significant. None of the infants had a positive aeroallergen screening test (Phadiatop).

The increase in plasma GLA level from baseline to three months of age was negatively and significantly associated with SCORAD at age one year (Table 5). The association was remarkably strong (odds ratio of 0.17 ; see also Table 6 ). No such association was found for $A D$ or total serum IgE (Table 5). The increase in plasma GLA level from baseline to six months was not related to any of the atopic outcomes.

Because the association between GLA increase and SCORAD could be biased (because it was based on an explanatory analysis), it may be subjected to protopathic bias. This bias exists when an early stage of disease (preceding the symptoms, hence 'protopathic') or an underlying etiological condition leads to changes of the risk factor, thus producing a spurious association between the risk factor and the disease. ${ }^{29}$ In the EFAtop study this may have occurred under the following conditions: 1) a genetic atopic constitution is truly associated with later high SCORAD and forms the underlying condition; 2) an atopic constitution increases the risk of food allergy in the first few months of life, and this is associated with feeding problems; 3 ) these feeding problems may lead to difficulty with the use of the supplement, indicated by non-compliance or sub-optimal compliance. The resulting lower intake of GLA is reflected in failure to show an increase in plasma levels of GLA. In this way a negative association between GLA-increase and SCORAD is produced, even if there is no true effect on SCORAD. We scrutinized the data in search of typical cases of infants who fulfilled these conditions for protopathic bias. As indicative of feeding problems related to food allergy we selected infants who switched to hypoallergenic formula, or who were suspected by the parents of having a food allergy, or had a positive food allergen test; and who showed feeding problems or were known as a non-complier. In these infants we evaluated whether SCORAD was particularly high (as a consequence of the underlying atopic condition) and if so, whether GLA increase was indeed low (as a consequence of possible non-compliance due to early atopic symptoms). This was done for infants in the verum group only, since only in this group does supplementation lead to GLA-increase. We identified only two infants fulfilling the conditions for protopathic bias. However, in these infants the SCORAD was in the middle range $(4.3-7.4)$ so that the impact on the results of the group as a whole can only be small and therefore we conclude that protopathic bias is not likely. 


\begin{tabular}{|c|c|c|c|c|c|c|}
\hline & GLA group $(n=51)$ & placebo group ( $n=51)$ & OR $(95 \% \mathrm{Cl})$ & $P$ & adjusted OR $(95 \% \mathrm{Cl})$ & $P$ \\
\hline Atopic dermatitis (UK-Working Party Probability Scores 0.50), $n$ & 19 & 22 & $0.78(0.35-1.73)$ & 0.54 & $0.81(0.36-1.82)$ & 0.61 \\
\hline Parents reported itch (present), $n$ & 3 & 5 & $0.58(0.14-2.55)$ & 0.47 & $0.50(0.11-2.30)$ & 0.37 \\
\hline Severity of atopic dermatitis (SCORAD), mean (SD) & $6.07(5.16)$ & $7.92(6.26)$ & b & 0.11 & $s$ & 0.06 \\
\hline Topical steroid use (present), n (class 1, class 2) & $1(1,0)$ & $4(4,0)$ & $0.24(0.03-2.18)$ & 0.20 & $0.24(0.03-2.24)$ & 0.21 \\
\hline Emollient use (present), $n$ & 24 & 17 & $1.78(0.80-3.96)$ & 0.16 & $1.68(0.73-3.86)$ & 0.22 \\
\hline Total serum lgE at age one year, geometric mean (SD) & $7.9(4.3)$ & $6.97(3.7)$ & 4 & 0.67 & - & 0.34 \\
\hline Positive food allergen test (Fx5), $n$ & $8(0$ missing $)$ & 4 (4 missing) & $2.05(0.57-7.32)$ & 0.27 & $2.48(0.67-9.16)$ & 0.87 \\
\hline
\end{tabular}

- Adjusted $O R=O R$ adjusted for total serum lgE at baseline (age one week)

B) Differences of the means between GLA group and placebo group $=-1.84$, confidence limits $-4.09 ; 0.41$ (studenf's t-test), $P=0.11$

s) Differences of the means between GLA group and placebo group corrected for baseline lgE (at one week) $=-2.25$, confidence limits $-4.57 ; 0.08$ (analysis of covariance), $P=0.06$

- Differences of the geometric means of total serum lgE between GLA and placebo groups $=1.12$, confidence limits $0.65 ; 1.93$ (student's t-test), $P=0.67$

- Differences of the geometric means of total serum lgE between GLA and placebo groups, corrected for baseline lgE (at one week) = 1.31, confidence limits 0.99 ; 1.25 (analysis of covariance), $P=0.34$

$O R=$ Odds Ratio

$\mathrm{Cl}=$ confidence interval 
Table 5

Risk of developing atopic dermatitis, depending on increase in plasma phospholipid GLA levels

\begin{tabular}{|c|c|c|}
\hline$A D$ (present ( $n=51$ ) vs, absent ( $n=67)$ ) & OR $(95 \% \mathrm{Cl}), P$ & $\begin{array}{l}\text { OR ( } 95 \% \mathrm{Cl} \text { ) when corrected } \\
\text { for covariables, } P\end{array}$ \\
\hline Increase in GLA between one week and three months & $0.81(0.40-1.65), 0.57$ & $1.11(0.47-2.65), 0.81$ \\
\hline Increase in GLA between one week and six months & $0.94(0.57-1.55), 0.81$ & $1.25(0.66-2.40), 0.49$ \\
\hline \multicolumn{3}{|l|}{ SCORAD (high ( $n=37)$ vs. low tertile $(n=38))$} \\
\hline Increase in GLA between one week and three months & $0.17(0.05-0.60), 0.01$ & $0.12(0.01-0.98), 0.048$ \\
\hline Increase in GLA between one week and six months & $0.62(0.30-1.29), 0.20$ & $0.72(0.24-2.19), 0.56$ \\
\hline \multicolumn{3}{|l|}{ IgE (high $(n=39)$ vs. low tertile $(n=39))$} \\
\hline Increase in GLA between one week and three months & $1.18(0.53-2.67), 0.68$ & $1.61(0.49-5.32), 0.43$ \\
\hline Increase in GLA between one week and six months & $1.22(0.68-2.18), 0.51$ & $1.34(0.59-3.00), 0.48$ \\
\hline
\end{tabular}

Odds ratio from logistic regression analysis calculated (with $95 \%$ confidence interval) for an increase in the plasma levels of GLA, comparing the 83.3 percentile and the 16.7 percentile in the whole group (corresponding with a contrast between higher and lower tertiles of GLA increase); this difference was 0.1023 weight\% GLA for the increase between 1 week and 3 months, and 0.0961 weight \% GLA for the increase between 1 week and 6 months. Contrast within SCORAD was assessed by dividing SCORAD into tertiles and testing high (high SCORAD, SCORAD range $8.7 \cdot 30.3$, mean 13.80 , SD 4.90 ) versus Iow (low SCORAD, SCORAD range $0-4.1$, mean $0.84, S D$ 1.62). Contrast within total serum lgE was assessed by dividing $\lg E$ into tertiles and testing high (high $\lg E$, lgE range 12.40 - 371.00, mean 59.94, SD 78.06) versus low (low lgE, lgE range $1-4.00$, mean 2.04, SD 1.02). Covariables included in the logistic regression were: atopic status of father, smoking during pregnancy, smoking by parents in the infants' first year, age of mother at time of delivery, $\lg E$ of infant at 1 week, $\lg E$ of the mother, number of siblings, day-care attendance, educational level of the father, educational level of the mother, presence of dog. presence of cat and use of allergen-free bed covers.

Table 6 Association between GLA increase in plasma phospholipids between one week and three months and SCORAD at one year of age

\begin{tabular}{lcccc}
\hline & \multicolumn{4}{l}{ Tertiles SCORAD at one year } \\
\cline { 2 - 5 } Tertiles GLA increase & $\begin{array}{c}\text { High tertile } \\
\text { (range } 8.7-30.3)\end{array}$ & $\begin{array}{c}\text { Middle tertile } \\
(4.2-8.6)\end{array}$ & $\begin{array}{c}\text { Low tertile } \\
(0-4.1)\end{array}$ & Total \\
\hline $\begin{array}{l}\text { High tertile } \\
\text { (range } 0.041-0.179)\end{array}$ & 7 & 13 & 17 & 37 \\
$\begin{array}{l}\text { Middle tertile } \\
\text { (range } 0.005-0.041)\end{array}$ & 12 & 17 & 10 & 39 \\
$\begin{array}{l}\text { Low tertile } \\
\text { (range }-0.216-0.005)\end{array}$ & 18 & 12 & 7 & 37 \\
Total & 37 & 42 & 34 & 113 \\
\hline
\end{tabular}

Spearman correlation coefficient $=-0.233(P=0.013)$

Linear by linear association $P=0.002$ 


\section{DISCUSSION}

As far as we know, this is the first published preventive trial of GLA supplementation on atopic dermatitis, as earlier trials have been therapeutic, aiming at improvement of existing atopic dermatitis. Results of these therapeutic trials have been inconsistent. ${ }^{30.31}$ Some of the trials had methodological drawbacks such as heterogeneity of the study population in terms of age, ${ }^{32}$ or in that either the subjects or the investigators were unblended. ${ }^{33}$ Among four trials in children with atopic dermatitis, three studies showed no effect of GLA vs. placebo ${ }^{11,34,35}$ and only one study ${ }^{10}$ showed a favorable effect on the severity of eczema of GLA compared to placebo. Our study was designed to investigate the possible protective role of GLA in developing atopic dermatitis in infants with atopic mothers.

Based on an intention-to-treat analysis, no significant beneficial effects of GLA supplementation on atopy were shown, although we observed a non-significant favorable trend for the clinical atopic outcomes (AD, SCORAD, itch and use of topical steroids) in the GLA group compared to the placebo group.

When the increase in plasma phospholipid GLA was used as a marker of compliance and intestinal uptake, the severity of atopic dermatitis as measured by the SCORAD was strongly negatively associated with the increase in GLA between one week and three months. Because this was no intention-to-treat analysis, it is important to check for biases. Firstly. selective follow-up was not likely to occur because all but three children completed the follow-up. Secondly, information bias was unlikely because the investigator, the patient and the outcome assessor remained blinded for the allocation of the supplements and for GLA levels in the infants. Thirdly, data were checked for protopathic bias, which was ruled out. Fourthly, when we controlled for possible confounding factors in the analyses, the association remained strong and significant. We therefore think that the association is real. However, this is not definite proof of a causal effect of supplementation; the results could also be explained by a metabolic difference in essential fatty acid metabolism between infants with and without an atopic constitution, which only comes to expression after GLA supplementation. However, this does not fit any of the current hypotheses. No associations were observed between the increase in GLA and AD or total serum IgE.

If there is a causal effect of GLA supplementation on severity of atopic dermatitis, the observation that the clearest results are related to the GLA levels in plasma at age three months would suggest that our constant dose of GLA over time might not have been sufficient with progressing age and increasing body weight. It is therefore possible that the effect of GLA would have been more pronounced with a higher dose of GLA at later age. Another explanation for these findings might be that this early period represents the time frame in which the immune system is most susceptible to GLA, which is consistent with the results of Galli et al. ${ }^{6}$ They demonstrated that the concentrations of $n-6$ LCPs were consistently and significantly lower in umbilical cord blood and in serum at one and three months of age in infants who developed atopy at the age of one year, compared to infants who remained unaffected, and that at one year of age these differences in n-6 LCP levels were no longer present.

Our study shows an effect of GLA on the severity of AD but not on the development of IgE at age one year. This indicates that GLA supplementation has a beneficial effect on the inflammatory component of atopic dermatitis, rather than on the $\mathrm{IgE}$-mediated component of atopic dermatitis. The absence of a correlation between clinical outcome measures (AD and 
SCORAD) and total and specific IgE in the present study also indicates that different components are implicated in the pathogenesis of atopic dermatitis. For a similar reason, it has recently been proposed to revise the nomenclature of atopic dermatitis, and refer to it as atopic eczema/dermatitis syndrome (AEDS), ${ }^{36}$ including IgE-mediated and non-IgE-mediated pathogenesis. The heterogeneity of patients in terms of AEDS between the previous therapeutic studies might partially explain the inconsistent results of GLA supplementation.

The beneficial effect of GLA supplementation on the severity of AEDS can be explained by the results of in vitro studies on skin epidermis. ${ }^{37,38}$ Normal skin epidermis is unable to convert LA into GLA. Dietary GLA is actively converted into DGLA in guinea pig epidermis, ${ }^{30}$ a model believed to resemble human epidermis. Because delta-5-desaturase, which converts DGLA into AA, is absent from skin epidermis, the increase in DGLA in the skin as result of, for instance, GLA supplementation will not result in an increase in AA and PGE2 in the skin. As a result, feeding humans a diet high in GLA raises PGE1 and 15-hydroxyeicosatrienoic acid (15-HETrE) levels. ${ }^{40}$ PGE1 and 15-HETrE both have anti-inflammatory properties. $^{37}$

We found a small but statistically significant higher level of in AA at three and six months of age in the GLA group compared to the placebo group. Since metabolites of AA are known to exert potent pro-inflammatory effects this can increase inflammation.' However, the results suggest that this is not the case given the positive effects found in the GLA group. The metabolic products of GLA and DGLA, PGE1 and 15-HetrE, have apparently been produced in sufficient amounts to inhibit the formation of these AA metabolites. ${ }^{40}$

Other studies provide additional explanations for the possible beneficial effects of EFAs on skin epidermis. Dry skin and itch are typical of patients with $A D$, and dry skin correlates with a disturbed epidermal barrier function. ${ }^{41}$ It has been shown that LA in particular is required for the formation and maintenance of the epidermal barrier. ${ }^{42,43}$ Also, topically applied evening primrose oil has yielded positive results in stabilizing the stratus corneum barrier ${ }^{44}$ and the most pronounced positive effect attributed to GLA in supplementation studies has been the reduction of itch. ${ }^{45-48}$ Besides the anti-inflammatory effects of GLA on skin epidermis, it might also play a physical structural role in the stability of the skin. Many of the immunological changes associated with GLA supplementation have been attributed to alterations in GLA metabolites that in turn downregulate the production of AA-derived leukotrienes. ' For instance, Ziboh and Fletcher showed that GLA inhibits LTB4 in a dosedependent way. ${ }^{40}$ The highest GLA dose in that study was very much comparable to the dosage used in our study (1500 mg GLA per day approximates $21 \mathrm{mg}$ per kg body weight per day for adults of about $70 \mathrm{~kg} ; 103 \mathrm{mg}$ GLA per day in our study approximates $19 \mathrm{mg}$ per kg body weight per day for infants of $5.5 \mathrm{~kg}$ at age 3 months). We also found a similar doseresponse relation. Therefore, we think that the results by Ziboh and Fletcher support our findings. However, to assess a cause-effect relationship, future preventive trials with GLAsupplementation should also focus on defining changes in metabolites involved in AD. In conclusion, the results show that early supplementation of GLA does not prevent the expression of atopy as reflected by total serum $\mathrm{IgE}$, but tends to alleviate the severity of atopic dermatitis in later infancy in children at high familial risk. Future studies should distinguish between atopic ( $\mathrm{gE}$-mediated) and inflammatory components of the atopic eczema/dermatitis syndrome. 


\section{ACKNOWLEDGEMENTS}

The authors would like to thank all midwives, parents and infants for participating, and Manon Meijs, Alice Fleuren, Annemie Mordant, Diane Crook, Hasibe Aydeniz, Nancy Hendrix, José Slangen, Janny de Vrieze and Steven Stapel for their valuable and enthusiastic assistance. The study was supported by grants form Roche Vitamins Europe Ltd (Basel, Switzerland) and Friesland Coberco Dairy Foods (Leeuwarden, The Netherlands).

\section{REFERENCES}

1. Kankaanpaa P, Sutas Y, Salminen S, et al. Dietary fatty acids and allergy. Ann Med. 1999;31:2827.

2. Brown WR, Hansen AE. Arachidonic and Linolic Acid of the serum in normal and eczematous human subjects. Proc Soc Exp Biol Med. 1937;36:113-117.

3. Manku MS, Horrobin DF, Morse N, et al. Reduced levels of prostaglandin precursors in the blood of atopic patients: defective delta-6-desaturase function as a biochemical basis for atopy. Prostaglandins Leukot Med. 1982;9:615-28.

4. Wright S, Sanders TA. Adipose tissue essential fatty acid composition in patients with atopic eczema. Eur J Clin Nutr. 1991;45:501-5.

5. Lindskov R, Holmer G. Polyunsaturated fatty acids in plasma, red blood cells and mononuclear cell phospholipids of patients with atopic dermatitis. Allergy 1992;47:517-21.

6. Galli E, Picardo M, Chini L, et al. Analysis of polyunsaturated fatty acids in newbom sera: a screening tool for atopic disease? Br J Dermatol. 1994;130:752-6.

7. Horrobin DF. Nutritional and medical importance of gamma-linolenic acid. Prog Lipid Res. 1992;31:163-94.

8. Wright $\mathrm{S}$, Bolton $\mathrm{C}$. Breast milk fatty acids in mothers of children with atopic eczema. $\mathrm{Br} \mathrm{J}$ Nutr. 1989;62:693-7.

9. Businco L, loppi M, Morse NL, et al. Breast milk from mothers of children with newly developed atopic eczema has low levels of long chain polyunsaturated fatty acids. J Allergy Clin Immunol. 1993:91:1134-9.

10. Bordoni A, Biagi PL, Masi M, et al. Evening primrose oil (Efamol) in the treatment of children with atopic eczema. Drugs Exp Clin Res. 1988;14:291-7.

11. Hederos CA, Berg A. Epogam evening primrose oil treatment in atopic dermatitis and asthma. Arch Dis Child. 1996;75:494-7.

12. Melnik B, Plewig G. Essential fatty acids, eicosanoids and postnatal T-cell maturation implications for treatment and prevention of atopy. J Dermatol Treat. 1994;5:157-161.

13. Jensen RG, Ferris AM, Lammi Keefe CJ. Lipids in human milk and infant formulas. Annu Rev Nutr. 1992;12:417-41.

14. Horrobin DF. Fatty acid metabolism in health and disease: the role of delta-6-desaturase. Am $J$ Clin Nutr. 1993:57:732s-736s.

15. Gibson RA, Kneebone GM. Fatty acid composition of human colostrum and mature breast milk. Am J Clin Nutr. 1981;34:252-7.

16. Otto SJ, Foreman van Drongelen MM, v. Houwelingen AC, et al. Effects of storage on venous and capillary blood samples: the influence of deferoxamine and butylated hydroxytoluene on the fatty acid alterations in red blood cell phospholipids. Eur J Clin Chem Clin Biochem. 1997;35:907-13.

17. Kaluzny MA, Duncan LA, Merritt MV, et al. Rapid separation of lipid classes in high yield and purity using bonded phase columns. J Lipid Res. 1985;26:135-40.

18. AI MD, van Houwelingen AC, Kester AD, et al. Maternal essential fatty acid patterns during normal pregnancy and their relationship to the neonatal essential fatty acid status. Br J Nutr. 1995;74:5568.

19. Paganelli R, Ansotegui IJ, Sastre J, et al. Specific IgE antibodies in the diagnosis of atopic disease. Clinical evaluation of a new in vitro test system, UniCAP, in six European allergy clinics. Allergy. 1998;53:763-8. 
20. Aalberse RC, Brummelhuis HG, Reerink Brongers EE. The purification of human polyclonal IgE by immunosorption. Immunochemistry. 1973;10:295-303.

21. Williams HC, Burney PG, Pembroke AC, et al. The U.K. Working Party's Diagnostic Criteria for Atopic Dermatitis. III. Independent hospital validation. Br J Dermatol. 1994;131:406-16.

22. Williams HC, Burney PG, Strachan D, et al The U.K. Working Party's Diagnostic Criteria for Atopic Dermatitis. II. Observer variation of clinical diagnosis and signs of atopic dermatitis. Br J Dermatol. 1994;131:397-405.

23. Kunz B, Oranje AP, Labreze L, et al. Clinical validation and guidelines for the SCORAD index: consensus report of the European Task Force on Atopic Dermatitis. Dermatology. 1997:195:10-9.

24. Severity scoring of atopic dermatitis: the SCORAD index. Consensus Report of the European Task Force on Atopic Dermatitis. Dermatology. 1993;186:23-31.

25. Chandra RK. Food allergy and food intolerance: lessons from the past and hopes for the 21st century. Bibl Nutr Dieta. 1991;48:149-56.

26. Hattevig G, Kjellman B, Sigurs N, et al. Effect of maternal avoidance of eggs, cow's milk and fish during lactation upon allergic manifestations in infants. Clin Exp Allergy. 1989;19:27-32.

27. Chandra RK. Prospective studies of the effect of breast feeding on incidence of infection and allergy. Acta Paediatr Scand. 1979;68:691-4.

28. Ruiz RG, Kemeny DM, Price JF. Higher risk of infantile atopic dermatitis from maternal atopy than from paternal atopy [see comments]. Clin Exp Allergy. 1992;22:762-6.

29. Feinstein A. Clinical Epidemiology. The architecture of clinical research. Philadelphia: WB Saunders Compagny, 1985.

30. Gamolenic acid in atopic eczema: Epogam. Drug Ther Bull.1990;28:69-70.

31. Hoare C, Li Wan Po A, Williams H. Systematic review of treatments for atopic eczema. Health Technol Assess. 2000;4:1-191.

32. Wright S. Atopic dermatitis and essential fatty acids: a biochemical basis for atopy? Acta dermato venereologica. Suppl. 1985;114:143-5.

33. Fiocchi A, Sala M, Signoroni P, et al. The efficacy and safety of gamma-linolenic acid in the treatment of infantile atopic dermatitis. J Int Med Res. 1994;22:24-32.

34. Bamford JT, Gibson RW, Renier CM. Atopic eczema unresponsive to evening primrose oil (linoleic and gamma-linolenic acids). J Am Acad Dermatol 1985;13:959-65.

35. Borrek S, Hildebrandt A, Forster J. Gammalinolensaure-reiche borretschsamenol-kapseln bei kindern mit atopischer dermatitis. Klinische Padiatrie 1997;209:100-104.

36. Johansson SG, Hourihane JO, Bousquet J, et al. A revised nomenclature for allergy: An EAACI position statement from the EAACI nomenclature task force. Allergy. 2001;56:813-824.

37. Ziboh VA, Miller CC, Cho Y. Metabolism of polyunsaturated fatty acids by skin epidermal enzymes: generation of antiinflammatory and antiproliferative metabolites. Am J Clin Nutr. 2000;71:361s-6s.

38. Ziboh VA. Prostaglandins, leukotrienes, and hydroxy fatty acids in epidermis. Semin Dermatol. 1992;11:114-20.

39. Miller CC, McCreedy CA, Jones AD, et al. Oxidative metabolism of dihomogammalinolenic acid by guinea pig epidermis: evidence of generation of anti-inflammatory products. Prostaglandins. 1988;35:917-38.

40. Ziboh VA, Fletcher MP. Dose-response effects of dietary gamma-linolenic acid-enriched oils on human polymorphonuclear-neutrophil biosynthesis of leukotriene B4. Am J Clin Nutr. 1992;55:3945.

41. Valsecchi R, Di Landro A, Pansera B, et al. Gammalinolenic acid in the treatment of atopic dermatitis. J Eur Acad Dermatol Venereol. 1996;7:77-79.

42. Schurer NY, Plewig G, Elias PM. Stratum corneum lipid function. Dermatologica 1991;183:77-94.

43. Hollmann J, Melnik BC, Lee MS, et al. Stratum-corneum- und Nagellipide bei Patienten mit atopischer Dermatitis. Verminderung der Ceramide-ein pathogenetischer Faktor bei atopischer Xerosis? Hautarzt 1991;42:302-6.

44. Gehring W, Bopp R, Rippke F, et al. Effect of topically applied evening primrose oil on epidermal barrier function in atopic dermatitis as a function of vehicle. Arzneimittelforschung 1999;49:635-42.

45. Andreassi M. Forleo P. Di Lorio A, et al. Efficacy of gamma -linolenic acid in the treatment of patients with atopic dermatitis. J Int Med Res. 1997;25:266-274.

46. Wright S, Burton JL. Oral evening-primrose-seed oil improves atopic eczema. Lancet. $1982 ; 2: 1120-2$. 
47. Schalin Karrila M, Mattila L, Jansen CT, et al. Evening primrose oil in the treatment of atopic eczema: effect on clinical status, plasma phospholipid fatty acids and circulating blood prostaglandins. Br J Dermatol. 1987;117:11-9.

48. Landi G. Oral administration of borago oil in atopic dermatitis. Joumal of Applied Cosmetology 1993;11:115-120.

49. Purasiri, P., A. McKechnie, et al. (1997). Modulation in vitro of human natural cytotoxicity, lymphocyte proliferative response to mitogens and cytokine production by essential fatty acids. Immunology 92(2): 166-72 



\title{
Determinants of neonatal IgE level - parity, maternal age, birth season and perinatal essential fatty acid status.
}

\author{
Christel J.A.W. van Gool \\ Carel Thijs' \\ Pieter C. Dagnelie' \\ Charles J.M. Henquet ${ }^{2}$ \\ Adriana C. v. Houwelingen ${ }^{3}$ \\ Jaap Schrander ${ }^{4}$ \\ Paul P.C.A. Menheere \\ Piet A. van den Brandt' \\ 'Department of Epidemiology. Maastricht University \\ 'Department of Dermatology, Maastricht University \\ 'Department of Human Biology, Maastncht University \\ 'Department of Pediatrics, Maastricht University \\ 'Department of Clinical Chemistry, University Hospital Masstricht
}




\begin{abstract}
OBJECTIVE

The hygiene hypothesis suggests that the protective 'siblings effect' against atopic diseases such as atopic dermatitis, allergic asthma and hay fever is a result of recurrent infections during early childhood. A recent study and review have indicated that this protective effect may already arise in utero. Lower n-3 essential fatty acid status is associated with increased parity, and essential fatty acid status has also been related to atopy. The present study tried to confirm the negative association between parity and neonatal IgE levels and further unravel the role of perinatal essential fatty acid status.
\end{abstract}

\title{
METHODOLOGY
}

In a prospective cohort study in 184 atopic mothers and their neonates, we simultaneously measured serum total $\mathrm{lgE}$ and essential fatty acid levels in plasma phospholipids, both in the mother at 34-36 weeks of gestation and in the neonate at the age of one week. Linear regression analysis was used to estimate the effect of parity on maternal and neonatal IgE and EFA status, and the independent effects of parity and essential fatty acid status on IgE, controlling for confounding factors such as maternal age and birth season.

\section{RESULTS}

Parity was associated with lower neonatal IgE level $(P<0.01)$, as well as with lower docosahexaenoic acid (DHA, 22:6n-3) status of the mother $(P=$ $0.01)$ but not of the neonate $(P>0.69)$. In the multivariate analysis, higher parity, higher maternal lgE, lower maternal age and birth in the first three months of the year were independently associated with neonatal lgE level. No association was detected between maternal or neonatal EFA status and neonatal IgE.

\section{CONCLUSIONS}

Since neonatal total serum $\mathrm{IgE}$ is predictive of later atopy, our results support the hypothesis that the sibling effect in atopy is already being programmed in utero. Our data also confirm earlier findings that DHA status is lower in multiparous women, but this did not confound the relation between parity and neonatal $\lg \mathrm{E}$. 


\section{INTRODUCTION}

The prevalence of atopic eczema in the Western world has increased two- to threefold over the last three decades.' Several theories have been put forward to explain this increase, most of them focusing on early life events. One example of such theories is the hygiene hypothesis. This was first postulated by Strachan ${ }^{2}$, who found the prevalence of hay fever and atopic dermatitis (AD) to be inversely related to the number of older siblings. Similar associations have been reported since. ${ }^{1.3,4}$ This effect has been tentatively explained by the greater chances of cross-infection from older siblings in large families, which may have an important protective role in the expression of atopy: the so-called sibling effect. The age of infection may be crucial, as it has also been found that $A D$ in the first year of life is related to the number of children in the household. ${ }^{5} \mathrm{~A}$ recent study by Karmaus ${ }^{6}$ suggested that cord blood IgE levels decrease with increasing birth order. As the concentration of total IgE in umbilical cord blood was found in several studies to be predictive of later development of atopy ${ }^{7.8}$, the sibling effect may have its origin (at least partly) in utero. If this hypothesis is correct, neonatal IgE would be expected to decrease with increasing parity.

Maternal EFA status is also influenced by parity, and has also been implicated in the development of atopy. It has long been recognized that atopic and non-atopic individuals differ with respect to the proportions of various polyunsaturated fatty acids in their blood. ${ }^{9}$ Studies in adults have found increased levels of linoleic acid $(18: 3 n-6$, LA) and alphalinolenic acid (18:3n-3, ALA), and decreased levels of n-6 and n-3 long chain polyenes (LCPs) in blood, adipose tissue and human milk of atopic individuals. ${ }^{10-13}$ The LCPs, n-6 and n-3 derivatives of LA and ALA, respectively, are the precursors of inflammatory mediators such as prostaglandines and leukotrienes and are embedded in cell membranes, where they play a role in membrane fluidity and signal transduction. An earlier study showed that low n-6 LCP levels in cord blood precede the development of atopy in infants ${ }^{14}$, which suggests a causal relationship and points to the importance of neonatal EFA status.

Neonatal EFA status depends on intrauterine EFA supply. Parity would, however, be expected to reduce the risk of atopic disease if maternal EFA status is depleted by earlier pregnancies. Increasing parity has indeed shown to be associated with relative n-3 LCP depletion, most notably of docosahexaenoic acid (DHA); maternal DHA status has been shown to decrease during a normal pregnancy ${ }^{15}$ and to decrease further with increasing parity. $^{16}$

We studied the effect of parity on neonatal total serum IgE levels and on perinatal DHA status, as well as their inter-independence, as part of an ongoing study of the relationship between the development of atopy and essential fatty acids. We also explored whether DHA or other perinatal EFAs are an intermediate or confounding factor in the relationship between parity and neonatal IgE.

\section{MATERIALS AND METHODS}

\section{STUDY DESIGN AND POPULATTON}

This study was initially designed to determine (1) the relationship in a group of breastfed neonates between the mothers' prepartum EFA status, EFA in breast milk, and the children's 
EFA status on the one hand and the development of atopic disease at the age of one year on the other; and (2) whether supplementing bottle-fed neonates with gamma-linolenic acid (GLA, 18:3n-6) in the first six months of life prevents atopic disease at the age of one year.

Subjects eligible for the study were atopic pregnant women and their neonates. They were recruited between October 1997 and April 2000 by midwives and via advertisements in local newspapers in the provinces of Limburg and Noord-Brabant (the Netherlands). The atopic status of both parents was assessed using a validated telephone questionnaire. Inclusion criteria for atopic mothers were: a history of allergic asthma or allergic rhino-conjunctivitis in relation to aeroallergen exposure, or a history of atopic dermatitis, or a history of positive allergy test, or improvement of asthma or mino-conjunctivitis complaints by antihistamine drugs. Exclusion criteria were: diabetes treated with medication and/or a prescribed diet, (pre-)-eclampsia and metabolic diseases.

Neonates were born between December 1997 and May 2000. Inclusion criteria for the neonates were: gestational age of at least 38 weeks, birth weight $>2500$ grams and an uncomplicated birth and neonatal period.

\section{SAMPLING AND LABORATORY ANALYSES}

Blood was collected from the mothers by venepuncture of the median cubital vein at 34-36 weeks of gestation, and from the infants at the age of about one week (5-8 days) by heel prick. Blood was collected in EDTA-containing tubes (Becton Dickinson, NJ USA) and serum separator tubes (Sherwood-Davis \& Geck, StLouis, USA). The samples were transported on ice/water mixture and, within 24 hours after collection, centrifuged for 10 minutes $(3000 \mathrm{~g}$ at $4^{\circ} \mathrm{C}$ ), and stored at $-20^{\circ} \mathrm{C}$ (serum) or $-50^{\circ} \mathrm{C}$ (plasma) under nitrogen until analysis. If an insufficient amount of venous blood was acquired from the mother, finger prick blood was collected on blotting paper for total serum IgE analysis. Neonatal blood sampling always preceded the start of the GLA supplementation.

Fatty acids (FAs) in plasma phospholipids were analyzed as previously described ${ }^{17}$ Briefly, lipid extracts were prepared from plasma samples, and phospholipid (PL) fractions separated using aminopropyl-bonded phase columns. ${ }^{18}$ PLs were hydrolyzed and FAs transmethylated with boron trifluoride (SIGMA) in methanol. The composition of the FA methyl esters obtained was determined by capillary gas chromatography using a polar capillary column (CPSil 88; Chrompack, Middelburg, the Netherlands), with helium as the carrier gas. The amount of each FA was quantified by adding an internal standard (dinonadecanoyl [19:0]-phosphatidylcholine, SIGMA). Results are reported as weight percentage (wt\%) of total FAs, computed as previously described. ${ }^{15}$

Specific IgE tests against a battery of food allergens ( $F \times 5)$, aeroallergens (Phadiatop) and a test for total serum IgE in the mother were performed in serum using the Pharmacia Unicap system (Pharmacia Upjohn, Uppsala, Sweden) as described elsewhere. ${ }^{19}$ Total IgE in the neonates was determined at the Central Laboratory of the blood-transfusion services in Amsterdam. This was done in eluded blood spot material in a sandwich assay, according to Aalberse et al. $^{20}$, with minor modifications: a mixture of anti-human $\lg \mathrm{E}$ monoclonal antibodies was coupled to Sepharose $4 \mathrm{~B}$ to catch $\lg \mathrm{E}$, and Sepharose-bound $\lg$ E was detected by means of radio-labeled antibodies against human IgE, raised in sheep. 


\section{STATISTTCAL ANALYSIS}

We evaluated three hypotheses: (1) parity influences maternal and neonatal total serum IgE, (2) parity influences DHA depletion in the mother during pregnancy and (3) the possible role of DHA as a confounder or as an intermediate factor in the relationship between parity and neonatal total serum IgE. In an explorative analysis, we assessed whether other EFAs were associated with neonatal IgE.

The main outcome variables for the first hypothesis were total serum IgE levels of mothers at 34 - 36 weeks of gestation (maternal $\mathrm{IgE}$ ) and of neonates at one week of age (neonatal $\lg E$ ). Total serum IgE was not normally distributed and was therefore transformed to its natural logarithm (InlgE). Linear regression analysis was applied to estimate the strength of the association between the outcome variables and parity, controlling for possible confounding factors (maternal age, season of birth, smoking during pregnancy and, in the case of the neonates, gender, maternal IgE, atopy of the father and type of feeding (formula or breast milk)).

The main outcome variables for the second hypothesis were matemal and neonatal DHA levels. Linear regression analysis was applied to estimate the strength of the association between these outcome variables and parity.

The main outcome variable for the third hypothesis was neonatal total serum $\lg E$ (InlgE). The possible role of DHA as a confounder or as an intermediate factor in the relationship between parity and neonatal total serum IgE was assessed by applying linear regression analysis to estimate the strength of the association between the outcome variables and parity, controlling for DHA in addition to the other possible confounding factors.

The explorative analysis assessed whether other EFAs were associated with neonatal total serum IgE, controlling for other covariables. The EFAs included in the analyses were LA $(18: 2 n-6)$, GLA (18:3n-6), dihomo-gammalinolenic acid (DGLA, 20:3n-6), arachidonic acid (AA, 22:4n-6), adrenic acid (22:4n-6), Osbond acid (22:5n-6), ALA (18:3n-3), 18:4n-3, eicosapentaenoic acid (EPA, 20:5n-3) and docosapentaenoic acid (DPA, 22:5n-3). To facilitate the interpretation of the regression coefficients when comparing the association of each EFA with $\operatorname{lgE}$, we computed standardized regression coefficients (= regression coefficient $x[\operatorname{SD}(x) / S D(y)]$, where $S D x$ is the standard deviation of the EFA and SDy is the standard deviation of LnigE in the study population). ${ }^{21}$

Statistical analyses were performed by means of the SPSS for Windows program (version 10.0, SPSS, Inc., Chicago, IL, USA).

\section{RESULTS}

\section{STUDY POPULATION}

One hundred and eighty-two mothers with their neonates were included in the study. As shown in table 1, almost half of the mothers were primipara (47\%), while $37 \%$ of the mothers had had one previous infant, $13 \%$ had had two previous infants and $3 \%$ had had three previous infants. Sixty-four neonates were exclusively breast-fed and 118 neonates were formula-fed in the first week. Seventy-nine percent of the mothers had a positive inhalant allergen test (Phadiatop) and $14 \%$ had a positive food allergen test (Fx5) (Table 1). 


\begin{tabular}{|c|c|c|c|c|c|c|c|c|}
\hline & \multicolumn{6}{|c|}{ Parity, $n(\%)$} & \multicolumn{2}{|l|}{ Total } \\
\hline & \multicolumn{2}{|l|}{1} & \multicolumn{2}{|l|}{2} & \multicolumn{2}{|l|}{$\geq 3$} & & \\
\hline & 85 & & 68 & & 29 & & 182 & \\
\hline maternal total serum IgE kU/L, mean (SD) & 356 & (728) & 214 & (451) & 146 & (191) & 269 & (578) \\
\hline $50-99, \mathrm{n}$ & 36 & & 36 & & 19 & & 91 & \\
\hline $100-199, n$ & 15 & & 11 & & 3 & & 29 & \\
\hline$>200, n$ & 34 & & 21 & & 7 & & 62 & \\
\hline maternal positive Phadiatopes, n (\%) & 64 & $(80 \%)$ & 53 & $(80 \%)$ & 20 & $(77 \%)$ & 137 & $(79 \%)$ \\
\hline maternal positive Fx $55^{\circ, 4}, \mathrm{n}(\%)$ & 16 & $(20 \%)$ & 8 & $(12 \%)$ & 2 & $(7 \%)$ & 26 & $(15 \%)$ \\
\hline smoking in pregnancy by mother, $\mathrm{n}(\%)$ & 18 & $(21 \%)$ & 8 & $(12 \%)$ & 5 & $(17 \%)$ & 31 & $(17 \%)$ \\
\hline paternal positive history of atopy, n (\%) & 30 & $(36 \%)$ & 26 & $(38 \%)$ & 10 & $(34 \%)$ & 66 & $(36 \%)$ \\
\hline age of mother at delivery, mean (SD) & 29 & (4) & 32 & (3) & 34 & (3) & 31.35 & (3.85) \\
\hline breastfeeding, $n(\%)$ & 28 & $(33 \%)$ & 27 & $(40 \%)$ & 9 & $(31 \%)$ & 64 & $(35 \%)$ \\
\hline \multicolumn{9}{|l|}{ Season of birth of neonate } \\
\hline 1tit (January, February, March) , n (\%) & 20 & $(24 \%)$ & 14 & $(21 \%)$ & 6 & $(21 \%)$ & 40 & $(22 \%)$ \\
\hline $2^{\text {nd }}($ April, May, June),$n(\%)$ & 17 & $(20 \%)$ & 21 & $(31 \%)$ & 11 & $(38 \%)$ & 49 & $(27 \%)$ \\
\hline $3^{\text {do }}$ (July, August, September) , n (\%) & 34 & $(40 \%)$ & 22 & $(32 \%)$ & 8 & $(28 \%)$ & 64 & $(35 \%)$ \\
\hline $4^{n}$ (October, November, December), n (\%) & 14 & $(16 \%)$ & 11 & $(16 \%)$ & 4 & $(14 \%)$ & 29 & $(16 \%)$ \\
\hline boys, $n(\%)$ & 36 & $(42 \%)$ & 28 & $(41 \%)$ & 22 & $(75 \%)$ & 86 & $(47 \%)$ \\
\hline infant $\lg E$ at one week kU/L, mean (SD) ${ }^{\circ}$ & \multicolumn{2}{|c|}{$1.7(3.9)$} & \multicolumn{2}{|c|}{$1.3(2.9)$} & \multicolumn{2}{|c|}{$0.34(0.5)$} & 1.32 & (3.26) \\
\hline non-detectable, $\mathrm{n}$ & \multicolumn{2}{|l|}{34} & \multicolumn{2}{|l|}{24} & \multicolumn{2}{|l|}{14} & \multicolumn{2}{|l|}{72} \\
\hline $0.2-0.5 \mathrm{kU} / \mathrm{L}, \mathrm{n}$ & \multicolumn{2}{|l|}{15} & \multicolumn{2}{|l|}{20} & \multicolumn{2}{|l|}{9} & \multicolumn{2}{|l|}{44} \\
\hline $0.5-18.0 \mathrm{kU} / \mathrm{L}, \mathrm{n}$ & \multicolumn{2}{|l|}{32} & \multicolumn{2}{|l|}{24} & \multicolumn{2}{|l|}{6} & \multicolumn{2}{|l|}{62} \\
\hline
\end{tabular}

$\mathrm{n}=$ number of participants

$\mathrm{SD}=$ standard deviation

Phadiatop = specific inhalant allergy test

Fx5 = food allergy test directed against cow's milk, egg-white, soy, shell-fish, nuts, wheat

a, b number of participants with positive specific test according to total serum IgE level

c, 24 mothers had parity 3,5 had parity 4

d, missing specific lgE in mothers $n=9$ (parity $1 n=5$, parity $2 n=2$, parity $\geq 3 n=2$ )

e, missing total $\lg E$ in infants $n=4$

\section{TOTAL SERUM IGE}

Increasing parity was associated with decreasing total serum IgE in the neonates ( $\mathrm{p}$-value = 0.006 ) (Table 2). The regression coefficient of -0.57 can be interpreted as follows: with each subsequent child, the neonatal IgE level increases by a factor $\exp (-0.57)=0.57(95 \%$ confidence limits $0.40 ; 0.85)$, i.e. the $\mathrm{lgE}$ level is almost halved. There was also a statistically significant effect of the age of the mother, suggesting that age is positively related to higher serum $\lg E$ levels in the children at the age of one week. Maternal IgE level showed no association with parity, but a borderline significant association with neonatal IgE. 
Table 2 Lnige in total serum of mother at $34-36$ weeks of gestation and in neonates at one week of age, in relation to parity and other determinants

\begin{tabular}{|c|c|c|c|c|c|c|}
\hline & \multicolumn{3}{|c|}{ Mother at $34-36$ weeks of gestation ( $n=182$ ) } & \multicolumn{3}{|c|}{ Neonate at one week of age (n=178") } \\
\hline & $\begin{array}{l}\text { regression- } \\
\text { coefficient }\end{array}$ & $\begin{array}{l}\text { standard } \\
\text { entor }\end{array}$ & Pvalue & $\begin{array}{l}\text { regression- } \\
\text { coefficient }\end{array}$ & $\begin{array}{l}\text { standard } \\
\text { error }\end{array}$ & Pvalue \\
\hline parity $(1,2,3,4)$ & -0.23 & 0.17 & 0.16 & -0.57 & 0.21 & 0.006 \\
\hline age of mother at delvery (years) & -0.06 & 0.04 & 0.08 & 0.10 & 0.04 & 0.02 \\
\hline Inige of mother (in kU/) & & & & 0.18 & 0.09 & 0.06 \\
\hline $\begin{array}{l}\text { smoking during pregnancy }(0=n 0,1=\text { yes }) \\
\text { season of birth }\end{array}$ & -0.42 & 0.31 & 0.17 & .0 .50 & 0.40 & 0.21 \\
\hline I" (January, Februay, March) & 0.15 & 0.31 & 0.64 & -1.68 & 0.46 & 0.001 \\
\hline $2^{\text {re }}$ (Apnil, May, June) & 0.72 & 0.32 & 0.02 & -0.67 & 0.46 & 0.15 \\
\hline $3^{n}$ (July, August, September) & 0.16 & 0.31 & 0.17 & -0.42 & 0.42 & 0.33 \\
\hline $4^{\text {th }}$ (October, November, December) & reference & & & reterence & & \\
\hline atopy tather $(0=n 0,1=$ yes $)$ & & & & 0.35 & 0.29 & 0.23 \\
\hline gender $(1=b o y, 2$ =girl) & & & & -0.21 & 0.29 & 0.46 \\
\hline ype of leeding ( $0=$ formula, 1 =breast mik $)$ & & & & 0.13 & 0.31 & 0.68 \\
\hline
\end{tabular}

Results of multivariate linear regression analysis.

.4 missing total serum lgE

\section{ROLE OF ESSENTIAL FATTY ACID STATUS}

Maternal DHA status decreased with parity (regression coefficient $=-0.33,(\mathrm{SE}=0.12), P=$ $0.007)$. Of the other EFAs, only EPA also decreased with parity $(-0.08(0.03), P=0.02)$. No effects of parity on DHA or other EFAs were found in the neonates at one week of age. When maternal DHA was included in the multivariate models of table 2, the regression coefficients of the association between parity and maternal or neonatal lgE hardly changed. This was also true when all other maternal or neonatal EFAs were included. Maternal DHA showed no statistically significant association with maternal $\mathrm{IgE}$ on its own (regression coefficient $=0.15(\mathrm{SE}=0.12), P=0.18)$, or with neonatal $\lg E(0.09(0.13), P=0.49)$.

The explorative analyses found a negative association between maternal LA levels and neonatal IgE levels $(P=0.04)$ (table 3 ). After correction for covariables, this association remained $(P=0.03)$ and $A A$ was positively associated with neonatal serum $\lg E$ levels. Neonatal ALA levels were negatively associated with neonatal $\lg E(P=0.03)$, while $18: 4 n-3$ and DPA were positively associated $(P=0.02$ and 0.03 , respectively). After correction for covariables, the association of ALA, 18:4n-3 and DPA with neonatal IgE remained statistically significant $(P=0.01,0.049$ and 0.04 , respectively) and EPA was negatively associated with neonatal $\operatorname{lgE}$ levels $(P=0.047)$. However, these associations showed no consistent pattern. 
Table 3. Neonatal InigE at one week of age, in relation to maternal phospholipid EFA status at $34-36$ weeks of gestation and neonatal phospholipid EFA status at 1 week of age and covariables

\begin{tabular}{|c|c|c|c|c|c|}
\hline \multirow{2}{*}{$\begin{array}{l}\text { Maternal phospholipid } \\
\text { EFAs (n=161) } \\
18: 2 n-6, \text { LA }\end{array}$} & \multicolumn{2}{|c|}{ Mean w\% (SD) } & \multicolumn{2}{|c|}{$\begin{array}{c}\text { Regression coefficient, } \\
\text { uncontrolled for covariables (SE) }\end{array}$} & \multirow{2}{*}{$\begin{array}{l}\text { Standardized coefficient, } \\
\text { uncontrolled for covariables } \\
-0.162\end{array}$} \\
\hline & 21.63 & (2.61) & -0.116 & $(0.057)$ & \\
\hline $18: 3 n-6$, GLA & 0.04 & $(0.03)$ & 4.143 & $(5.364)$ & 0.062 \\
\hline $20: 3 n-6$, DGLA & 3.53 & $(0.70)$ & -0.151 & $(0.213)$ & -0.057 \\
\hline $20: 4 n-6, A A$ & 8.22 & $(1.49)$ & 0.172 & $(0.099)$ & 0.137 \\
\hline $22: 4 n-6$, adrenic acid & 0.37 & $(0.23)$ & 0.240 & $(0.654)$ & 0.029 \\
\hline $22: 5 n-6$, Osbond acid & 0.45 & $(0.16)$ & 0.722 & $(0.937)$ & 0.062 \\
\hline 18:3n-3, ALA & 0.25 & $(0.09)$ & -1.523 & (1.683) & -0.072 \\
\hline $18: 4 n-3$ & 0.12 & $(0.05)$ & -2.597 & (3.030) & -0.068 \\
\hline 20:5n-3, EPA & 0.34 & $(0.28)$ & 0.959 & (0.535) & 0.142 \\
\hline $22: 5 n-3$, DPA & 0.55 & $(0.13)$ & 1.413 & (1.179) & 0.096 \\
\hline $22: 6 n-3$, DHA & 3.79 & $(1.06)$ & 0.167 & $(0.140)$ & 0.095 \\
\hline \multicolumn{6}{|l|}{ Neonatal phospholipid } \\
\hline \multicolumn{6}{|l|}{ EFAs $(n=178)$} \\
\hline $18: 2 n-6$, LA & 16.57 & $(2.41)$ & -0.020 & $(0.060)$ & -0.025 \\
\hline $18: 3 n-6$, GLA & 0.07 & $(0.05)$ & -4.220 & (3.148) & -0.101 \\
\hline $20: 3 n-6$, DGLA & 2.67 & $(0.72)$ & 0.141 & (0.202) & 0.053 \\
\hline $20: 4 n-6, A A$ & 11.34 & (3.03) & 0.063 & $(0.048)$ & 0.100 \\
\hline $22: 4 n-6$, adrenic acid & 0.37 & $(0.12)$ & 1.580 & (1.248) & 0.096 \\
\hline $22: 5 n-6$, Osbond acid & 0.34 & $(0.21)$ & -0.206 & $(0.681)$ & -0.023 \\
\hline 18:3n-3, ALA & 0.11 & $(0.08)$ & -3.748 & (1.758) & -0.160 \\
\hline $18: 4 n-3$ & 0.06 & $(0.04)$ & 8.554 & (3.612) & 0.177 \\
\hline 20:5n-3, EPA & 0.32 & $(0.14)$ & -1.227 & $(1.004)$ & -0.093 \\
\hline $22: 5 n-3$, DPA & 0.32 & $(0.14)$ & 2.243 & (1.052) & 0.160 \\
\hline $22: 6 n-3$, DHA & 3.87 & (1.18) & 0.120 & $(0.124)$ & 0.122 \\
\hline
\end{tabular}

Regression coefficients uncontrolled for covariables are between one particular EFA and neonatal InigE.

Regression coefficients controlled for covariables are between one particular EFA and neonatal InlgE, controlled for the model presented in table 2.

$\mathrm{SD}=$ standard deviation, $\mathrm{SE}=$ standard error, $\mathrm{wt} \%$ = weight percentage total fatty acids

\section{DISCUSSION}

The first aim of the present study was to evaluate the association between parity and neonatal lgE levels. Our results confirm Karmaus findings ${ }^{6}$ that parity is related to lower lgE levels in neonatal serum. Other determinants of neonatal IgE level were found to be higher maternal $\operatorname{lgE}$, higher maternal age and birth outside the first three months of the year.

Our second hypothesis concerned the association between parity and maternal and neonatal EFA status, especially DHA. Other studies have shown that parity is associated with lower maternal DHA status and also with neonatal EFA status as measured in cord plasma and umbilical vein and artery wall. ${ }^{16}$ Our data confirm that maternal DHA decreases with increasing parity, but neonatal DHA was unrelated to parity. There are several potential explanations for our findings. Firstly, there may be mechanisms ensuring neonatal EFA status at the expense of maternal EFA status. Secondly, the neonates may be able to synthesize a sufficient amount of DHA from its precursors soon after birth and normalize a DHA deficit within one week. Current evidence is indecisive for these explanations. ${ }^{22,23}$ 


\begin{tabular}{|c|c|c|c|c|}
\hline Pvalue & $\begin{array}{r}\text { Regression } \\
\text { controlled for oc }\end{array}$ & $\begin{array}{l}\text { coefficient. } \\
\text { ovariables (SE) }\end{array}$ & $\begin{array}{l}\text { Standardzed coefficient. } \\
\text { controlled for covariables }\end{array}$ & Avalue \\
\hline 0.04 & -0.129 & 1.845 & -0.180 & 0.02 \\
\hline 0.44 & 4.236 & 5.311 & 0.063 & 0.42 \\
\hline 0.48 & -0.166 & 0.211 & -0.062 & 0.43 \\
\hline 0.09 & 0.212 & 0.097 & 0.169 & 0.03 \\
\hline 0.71 & 0.167 & 0.634 & 0.020 & 0.79 \\
\hline 0.44 & 0.749 & 0.956 & 0.063 & 0.43 \\
\hline 0.37 & -1.237 & 1.702 & -0.059 & 0.47 \\
\hline 0.39 & -3.986 & 3.075 & -0.105 & 0.20 \\
\hline 0.08 & 0.539 & 0.539 & 0.080 & 0.32 \\
\hline 0.23 & 1.068 & 1.198 & 0.072 & 0.37 \\
\hline 0.24 & 0.084 & 0.143 & 0.047 & 0.56 \\
\hline 0.74 & 0.009 & 0.063 & 0.011 & 0.89 \\
\hline 0.18 & -4.709 & 3.051 & -0.113 & 0.13 \\
\hline 0.49 & -0.062 & 0.251 & -0.023 & 0.80 \\
\hline 0.19 & 0.020 & 0.063 & 0.031 & 0.75 \\
\hline 0.21 & 1.410 & 1.324 & 0.085 & 0.29 \\
\hline 0.76 & -0.632 & 0.653 & -0.071 & 0.33 \\
\hline 0.03 & -4.747 & 2.110 & -0.203 & 0.03 \\
\hline 0.02 & 7.299 & 3.674 & 0.151 & 0.049 \\
\hline 0.22 & -1.942 & 0.972 & -0.145 & 0.047 \\
\hline 0.03 & 2.469 & 1.206 & 0.175 & 0.04 \\
\hline 0.11 & 0.088 & 0.126 & 0.053 & 0.49 \\
\hline
\end{tabular}

Our third hypothesis was that DHA and other EFAs play a role in the relationship between parity and neonatal IgE levels. No relationship was found between maternal DHA and neonatal IgE levels. We assessed the role of maternal DHA in the relationship between parity and neonatal IgE by including maternal DHA in the multivariate analysis. Since the relationship between parity and neonatal IgE did not change, we conclude that maternal DHA is not a confounding or intermediate factor in this relationship. Since this was also true when all EFAs were included in the model together, it is not likely that EFA status or any particular EFAs are confounding or intermediate factors.

The above relationships were studied in a hypothesis-driven approach. In a further analysis we explored whether other perinatal EFAs might be related to neonatal IgE levels. We found several associations that can only be interpreted in the light of specific hypotheses. There are currently two main hypotheses to explain the relationship between EFAs and atopy. The first hypothesis suggests that desaturation of LA and ALA into their longer chain polyenes (LCPs) is impaired in atopic subjects as a result of an impaired enzyme activity of delta-6desaturase (the delta-6-desaturase hypothesis). ${ }^{10.24}$ This hypothesis is based observational studies that found increased levels of LA and substantially lower levels of its LCPs, such as $A A$, in blood ${ }^{10-12}$, adipose tissue 11 and breast milk ${ }^{13}$ of adults with atopic disease, as 
compared to healthy subjects. As regards the n-6 EFAs, we did not confirm a higher LA level in mother or neonate in relation to high neonatal IgE (in fact, the reverse was found for maternal LA). Nor did we find lower n-6 LCPs, and maternal AA was even higher in relation to high neonatal IgE. As regards the n-3 EFAs, no association was found between maternal EFAs and neonatal IgE. The associations found for neonatal n-3 EFAs showed no pattern consistent with the delta-6-desaturase hypothesis.

The second hypothesis is based on the time trends in the prevalence of atopic diseases and the consumption of fatty acids in developed countries. The increased consumption of vegetable oils rich in LA and a simultaneously reduced consumption of fatty fish (rich in n-3 EFAs) has been accompanied by an increased prevalence of atopic disease. ${ }^{25.26}$ It has therefore been hypothesized that the increased consumption of LA and the consequent increase in the ratio between n-6 and n-3 EFAs in the diet may have led to an increase in atopic diseases (the n-6/n-3 EFA hypothesis). We found no consistently higher n-6 and lower $\mathrm{n}-3$ EFA levels in relation with neonatal $\mathrm{IgE}$. We conclude that none of the relationships we found were in agreement with any of the hypotheses on EFAs and atopy, and that none of the changes showed a consistent pattern. Nevertheless, the number of statistically significant associations between neonatal n-3 EFAs and neonatal IgE was higher than expected on the basis of chance and this topic therefore needs further research and perhaps a different hypothesis.

Since neonatal IgE is thought to be a determinant of later atopy, the present results seem to contradict the known relationship between perinatal EFAs and the later development of atopy. 14, 27, ${ }^{28}$ However, most studies used clinical atopic outcomes or composite outcomes including clinical and laboratory data; the study in infants that showed an association between cord blood EFAs and later atopy did not find an association with later IgE either. ${ }^{14}$ Further evidence that EFAs are not related to the IgE component of atopy was found in the present study (results presented elsewhere). We found that a relatively low perinatal n-3 LCP status in the breast-fed infants was associated with greater severity of atopic dermatitis at age one year, but not with total serum $\lg E$ at that age. In a supplementation study with GLA we found that the increase in plasma GLA during supplementation was associated with lower severity of atopic dermatitis, but again not with total serum $\lg \mathrm{E}$. We conclude that the effect of perinatal EFAs on the later development of atopic disease must be independent of the neonatal production of $\lg \mathrm{E}$ and is not likely to be influenced by parity.

Karmaus has provided several hypothetical explanations for the possible in utero effects of parity on total serum IgE levels in cord blood ${ }^{29}$ and summarized these explanations as the 'in utero programming disruption hypothesis'. Changes in hormones, maternal immune suppression to prevent rejection of the fetus, maternal infections during pregnancy and lifestyle characteristics such as smoking and employment might all influence the environment of the unborn child. One of two North-European studies found blood IgE levels to be associated with parental smoking during pregnancy ${ }^{30}$, whereas the other found an association with maternal caffeine and alcohol use. ${ }^{31}$

A large German study showed no association between birth season and cord blood IgE, and two North-European studies were contradictory, one showing peaks in late autumn ${ }^{31}$ whereas the other showed the lowest cord blood values in autumn. ${ }^{32}$ We know of no previous studies that have reported on the seasonality of both maternal and neonatal $\mathrm{lgE}$. Our finding of higher maternal IgE levels in spring is consistent with pollen allergy. To match this with our finding of lowest neonatal IgE values in the first three months of the year, it is 
important to recognize that the fetal ability to produce $\lg E$ does not start before the second trimester. ${ }^{33}$ Our finding of lowest neonatal IgE values in the first three months of the year is consistent with second and third trimester pregnancy outside the pollen season. Therefore, our findings suggest that pollen exposure in pregnant women with a personal history of atopy confers intrauterine stimulation of fetal $\mathrm{IgE}$ production. In a review of intrauterine influences on atopy, Jones concluded that allergens but not $\mathrm{IgE}$ are transferred through the placenta, and proposed that $\mathrm{lgE}$ is amniotically transferred and ingested by the fetus, and is later counterbalanced by transplacental $\operatorname{lgG} .^{33}$ If neonatal $\operatorname{lgE}$ originated directly from the mother, it would show a peak simultaneously with that of the mother, which it did not in our study. Therefore, possible explanations include fetal production of $\lg E$ stimulated by maternally transferred pollen allergens, transamniotic IgE transfer or unknown matemal immunologic stimuli related to seasonal allergy.

The present study confirms the conclusions of earlier studies: parity has a negative influence on the DHA status in pregnant mothers and is also related to total serum $\operatorname{lgE}$ in neonates at the age of one week. However, our results show that the effect is independent of changes in EFA status.

\section{ACKNOWLEDGEMENTS}

The authors would like to thank all midwives, parents and infants for participating: Manon Meijs, Alice Fleuren, Annemie Mordant, Diane Crook, Hasibe Aydeniz, Nancy Hendrix, Janny de Vrieze and Steven Stapel provided valuable and enthusiastic assistance. The study was supported by grants form Roche Vitamins Europe Ltd (Basel, Switzerland) and Friesland Coberco Dairy Foods (Leeuwarden, The Netherlands).

\section{REFERENCES}

1. Williams HC. Is the prevalence of atopic dermatitis increasing? Clin Exp Dermatol. 1992;17:38591.

2. Strachan DP. Hay fever, hygiene, and household size. BMJ. 1989;299:1259-60.

3. von Mutius E, Martinez FD, Fritzsch C, Nicolai T, Reitmeir P. Thiemann HH. Skin test reactivity and number of siblings. BMJ. 1994;308:692-5.

4. Matricardi PM, Rosmini F, Ferrigno L, et al. Cross sectional retrospective study of prevalence of atopy among Italian military students with antibodies against hepatitis A virus. BMJ. 1997;314:999-1003.

5. Strachan DP. Family size, infection and atopy: the first decade of the "hygiene hypothesis". Thorax. 2000;55:1s2-10.

6. Karmaus W, Arshad H, Mattes J. Does the sibling effect have its origin in utero? Investigating birth order, cord blood immunoglobulin $\mathrm{E}$ concentration, and allergic sensitization at age 4 years. Am J Epidemiol. 2001:154:909-15.

7. Martinez FD, Wright AL, Taussig LM, et al. Asthma and wheezing in the first six years of life. The Group Health Medical Associates. N Engl J Med. 1995;332:133-8.

8. Chandra RK, Puri S, Cheema PS. Predictive value of cord blood IgE in the development of atopic disease and role of breast-feeding in its prevention. Clin Allergy. 1985;15:517-22.

9. Brown WR, Hansen AE. Arachidonic and Linolic Acid of the serum in normal and eczematous human subjects. Proc Soc Exp Biol Med. 1937;36:113-117.

10. Manku MS, Horrobin DF, Morse N, et al. Reduced levels of prostaglandin precursors in the blood of atopic patients: defective delta-6-desaturase function as a biochemical basis for atopy. Prostaglandins, Leukotrienes and Medicine 1982;9:615-28. 
11. Wright S, Sanders TA. Adipose tissue essential fatty acid composition in patients with atopic eczema. Eur J Clin Nutr. 1991;45:501-5.

12. Lindskov R, Holmer G. Polyunsaturated fatty acids in plasma, red blood cells and mononuclear cell phospholipids of patients with atopic dermatitis. Allergy. 1992;47:517-21.

13. Thijs C, Houwelingen A, Poorterman I, et al. Essential fatty acids in breast milk of atopic mothers: comparison with non-atopic mothers, and effect of borage oil supplementation. Eur $\mathrm{J}$ Clin Nutr. 2000;54:234-8.

14. Galli E, Picardo M, Chini L, et al. Analysis of polyunsaturated fatty acids in newborn sera: a screening tool for atopic disease? Br J Dermatol. 1994;130:752-6.

15. AI MD, van Houwelingen AC, Kester AD, et al. Maternal essential fatty acid patterns during normal pregnancy and their relationship to the neonatal essential fatty acid status. Br J Nutr 1995;74:5568.

16. Al MD, van Houwelingen AC, Hornstra G. Relation between birth order and the maternal and neonatal docosahexaenoic acid status. Eur J Clin Nutr. 1997;51:548-53.

17. Otto SJ, Foreman van Drongelen MM, v. Houwelingen AC, et al. Effects of storage on venous and capillary blood samples: the influence of deferoxamine and butylated hydroxytoluene on the fatty acid alterations in red blood cell phospholipids. Eur J Clin Chem Clin Biochem. 1997;35:907-13.

18. Kaluzny MA, Duncan LA, Merritt MV, et al. Rapid separation of lipid classes in high yield and purity using bonded phase columns. J Lipid Res. 1985;26:135-40.

19. Paganelli R, Ansotegui IJ, Sastre J, et al. Specific IgE antibodies in the diagnosis of atopic disease. Clinical evaluation of a new in vitro test system, UniCAP, in six European allergy clinics. Allergy. 1998;53:763-8.

20. Aalberse RC, Brummelhuis HG, Reerink Brongers EE. The purification of human polyclonal IgE by immunosorption. Immunochemistry. 1973;10:295-303.

21. Kleinbaum DG, Kupper LL, Muller KE, et al. Applied regression analysis and other multivariable methods. Pacific Grove: Duxbury Press, 1998.

22. Innis SM, Sprecher H, Hachey D, et al. Neonatal polyunsaturated fatty acid metabolism. Lipids. 1999:34:139-49.

23. Hornstra G. Essential fatty acids in mothers and their neonates. Am J Clin Nutr 2000:71:1262s-9s.

24. Horrobin DF. Nutritional and medical importance of gamma-linolenic acid. Prog Lipid Res. 1992;31:163-94.

25. Black PN. Sharpe S. Dietary fat and asthma: is there a connection? Eur Respir J. 1997;10:6-12.

26. Kankaanpaa P, Sutas Y, Salminen S, et al. Dietary fatty acids and allergy. Ann Med. 1999;31:2827.

27. Wright S, Bolton C. Breast milk fatty acids in mothers of children with atopic eczema. Br J Nutr. 1989;62:693-7.

28. Businco L, loppi M, Morse NL, et al. Breast milk from mothers of children with newly developed atopic eczema has low levels of long chain polyunsaturated fatty acids. J Allergy Clin Immunol. 1993;91:1134-9.

29. Karmaus W, Botezan C. Does a higher number of siblings protect against the development of allergy and asthma? A review. J Epidemiol Community Health. 2002;56:209-17.

30. Bergmann RL, Schulz J, Gunther S, et al. Determinants of cord-blood IgE concentrations in 6401 German neonates. Allergy. 1995;50:65-71.

31. Bjerke T, Hedegaard M, Henriksen TB, et al. Several genetic and environmental factors influence cord blood IgE concentration. Pediatr Allergy Immunol. 1994;5:88-94.

32. Hansen LG, Host A, Halken S, et al. Cord blood IgE. I. IgE screening in 2814 newborn children. Allergy. 1992;47:391-6.

33. Jones CA, Holloway JA, Wamer JO. Does atopic disease start in foetal life? Allergy. 2000;55:210. 
Oral essential fatty acid supplementation in atopic dermatitis - a meta analysis of placebo controlled trials

Christel J.A.W. van Gool

Maurice P.A. Zeegers

Carel Thijs

Department of Epidemiology. Maastricht University 


\begin{abstract}
BACKGROUND

Essential fatty acids are components of cell membranes and precursors of immunomodulating factors that may play a role in the inflammatory and immunological pathogenesis of atopic dermatitis. Trials of supplementation with essential fatty acids to alleviate atopic dermatitis have given inconsistent results.
\end{abstract}

\title{
OBJECTTVE
}

To summarize and quantify the results of placebo controlled trials with essential fatty acids.

\section{DESIGN}

Publications on clinical trials were searched in a systematic way and assessed independently by three assessors on study characteristics. Trials were selected for inclusion in the meta-analysis when they had included a placebo group and when the outcome measure included (severity of) atopic dermatitis. Pooled effect sizes of improvement of overall severity of $A D$ were calculated by random effects meta-analysis. The dependency of the results on study characteristics was studied using meta-regression analysis.

\section{RESULTS}

We identified 34 publications on clinical studies up to April 2002. Nineteen trials of GLA and 5 trials of fish oil matched our inclusion criterion of placebo-controlled trial. The effect size of GLA supplementation on improvement of overall severity of atopic dermatitis could be calculated from 11 trials. The pooled effect size was 0.15 (95\% confidence limits $0.02 ; 0.32$ ). The effect size of fish oil supplementation, calculated from 4 trials was $-0.06(95 \% \mathrm{CL}-0.32 ; 0.21)$. For component subscales such as itch, scaling and lichenification, EFA supplementation showed no benefit. Study characteristics showed no detectable influence on the overall result.

\section{CONCLUSION}

Supplementation of EFA has no clinically relevant effect on the severity of $\mathrm{AD}$. 


\section{INTRODUCTION}

The frequency of atopic dermatitis (AD) has increased in industrialised countries over the past few decades;' e.g. in Denmark the cumulative incidence of AD in children at the age of $0-7$ years was $2 \%$ in the early 1960 s, about $10 \%$ in the early 1970 s and about $12 \%$ in $1987 .{ }^{2}$ Essential fatty acids might play a role in the aetiology of AD. Trials of oral essential fatty acid (EFA) supplementation for atopic dermatitis have given inconsistent results. ${ }^{3.4}$ In this paper we review these trials in a systematic manner, attempting to explain the inconsistencies by differences in study populations, supplements and study characteristics.

In 1937, Brown \& Hansen detected decreased levels of arachidonic acid $(20: 4 n-6, A A)$ in serum of children with $A D .^{5}$ More recent observational studies have confirmed abnormal $n-6$ fatty acid levels in patients with $A D .^{6-9}$ Early studies suggest that linoleic acid $(18: 2 n-6, L A)$ is insufficiently converted into gamma-linolenic acid (18:3n-6, GLA) and further elongated and more unsaturated products, the so-called long chain polyenes (LCPs) ${ }^{10}$ Manku and Horrobin, ${ }^{11,12}$ amongst others, defined the hypothesis that the fatty acid profiles in subjects with atopic dermatitis reflect a lower activity of delta-6-desaturase (D6D), the enzyme determining the rate-limiting step in the desaturase-elongase pathway of EFAs. This enzyme desaturates LA in into GLA and alpha-linolenic acid into 18:4n-3 and is the first step of the elongation and desaturation pathway of EFAs. The resulting LCPs are precursors of immunomodulating factors such as prostaglandins and leukotrienes. GLA is the precursor of inflammatory mediators such as prostaglandin E1 (PGE1), and these influence AD mainly through two pathways: firstly, PGE1 is involved in the maturation and differentiation of Tlymphocytes and thus might influence $\lg \mathrm{E}$ production. ${ }^{13}$ Secondly. GLA supplementation might alleviate the inflammatory component of AD. Normal skin epidermis is unable to convert LA into GLA and also delta-5-desaturase is absent in human skin. As a result, DGLA is not further elongated into AA. When humans are consuming a diet high in GLA, its metabolic products PGE1 and 15-hydroxy-eicosatrienoic acid (15-HETrE) levels will rise in the skin. Both products have anti-inflammatory properties. ${ }^{14-16}$ If GLA supplementation alleviates the inflammatory component of $A D$, the effect may be most pronounced in $A D$ patients with active inflammation.

Sources of GLA are evening primrose oil, black current oil and borage oil. Trials of GLA supplementation with these oils of patients with AD have been performed since the $1980 \mathrm{~s}$ with varying quality and varying results. Although some narrative reviews have been published, ${ }^{17-21}$ only one systematic review ${ }^{22}$ has been conducted. This meta-analysis dating from 1989 included published and unpublished studies but has been criticized for lack of sufficient detail about the unpublished trials, e.g. on baseline measurements. ${ }^{23.24}$ Furthermore, one of the co-authors of this meta-analysis was affiliated to the manufacturer of one of the commercially available supplements with GLA. The large published trial by Bamford et al. $^{3}$ that gave negative results was rejected from the meta-analysis with the allegation from its authors (one of whom was also responsible for the fatty acid measurements of the Bamford study) that "both groups showed a significant elevation of DGLA and AA. [...] It can be concluded that in the Bamford trial some of the patients assigned to receive placebo actually received active treatment and vice versa." ${ }^{22}$ This has never been refuted in the literature until recently this question was raised again in a larger 
review and the allegations were denied by Bamford. ${ }^{25}$ It is remarkable that also another large negative trial, ${ }^{26}$ after publication was attacked on its design by the sponsor, ${ }^{27}$ who had also sponsored the Bamford trial. Furthermore, a meta-analysis by the UK Department of Health of published and unpublished trials of evening primrose oil supplementation for AD has been conducted, but was not published because the authors did not get permission to publish the data. ${ }^{25}$

Also n-3 EFAs are thought to be effective in improving symptoms of $A D$ because the products of n-3 EFAs such as leukotrienes of the 5-series and prostaglandins of the n-3 series are less pro-inflammatory than the n-6 products. As a result, some trials have focused on fish oil supplementation (rich in n-3 EFAs) vs placebo in AD.

We performed a new meta-analysis because the last one $e^{22}$ was performed thirteen years ago and many more controlled clinical trials have been published since. Furthermore, we wanted to use a more systematic approach. We wondered whether inconsistencies in results between studies might be explained by differences in characteristics of supplements, differences in characteristics of the study population and publication bias. Characteristics of the supplements were its formulation (GLA and n-3 LCPs) and the placebo, and the duration of supplementation, since sufficient time is needed to incorporate EFAs in the target tissues. Characteristics of study populations were degree of inflammation or severity of eczema (because of the anti-inflammatory action of GLA and n-3 LCPs) and age of the patients. In infants, Galli et al. $(1994)^{28}$ showed that the aberrant EFA profiles preceded the development of $A D$. If, as suggested, GLA plays a role in the early development of $A D, G L A$ supplementation might be of benefit to young children with recent onset of $A D$, but not in adult patients with longstanding disease. Publication bias may be identified if industryrelated trials show more positive results compared to un-sponsored trials, and if negative studies with low statistical precision are relatively lacking among publications. ${ }^{29}$

\section{MATERIALS AND METHODS}

\section{SEARCH STRATEGY}

Publications were identified through computerized MEDLINE, EMBASE and Current Contents searches for controlled trials published from 1966 until April 2002 with no language restriction. The keywords used were: efamol, epogam, glandol, linolenic, gamma linolenic acid, gammolenic, borage, borago, evening primrose, oenothera, blackcurrant or blackcurrant; and neurodermitis, atop*, dermat* or eczem*. Furthermore, references cited in published original and review articles were examined until no further study was identified. Controlled trials were selected if patients with $A D$ were included; the design was a parallel or cross-over-controlled trial; treatment consisted of supplementation with essential fatty acids and placebo was used as a control. If randomisation was not mentioned in the publication we considered it to be a non-randomised clinical controlled trial (CCT). 


\section{QUALITATTVE DATA EXTRACTION}

If different publications on identical studies were found, we combined these to extract the maximum amount of information.

All three of us reviewed the characteristics of the studies. Two of us (MZ, CT) were blinded for year of publication, authors, affiliations and funding, and results. By means of a standardized checklist we independently assessed the following items: inclusion and exclusion criteria, characteristics of study population, baseline comparability of groups reported, blinding of patient and outcome assessor, compliance, co-supplements, loss-tofollow-up, outcome parameters and whether the analysis was intention-to-treat. If disagreement existed on an item it was discussed through consensus meetings. In addition, one of us (CvG) recorded the composition and dosage of the active and placebo supplements, and the duration of supplementation.

Signs of competing interest as explanation for possible publication bias were checked for in the original articles, letters to the editor and commentaries. As markers of competing interest we considered affiliations of the authors with the manufacturer (grade 3); funding by manufacturers of EFA supplements (or alternatively, independent or non-profit organizations) (grade 2); and sponsorship through donation of commercially available EFA supplements for use in the trial (grade 1). If none of these markers were present it was graded 0.

\section{QUANTTTATTVE DATA EXTRACTION}

We considered severity of $A D$ as the major outcomes of the studies, such as a score on a previously published scale (such as COSTA-score), the judgment of the physician who assessed the patients at baseline and at follow-up and/or the patient's or parents' selfreported overall improvement. If also no overall score was given, we summarized all the reported subscales (such as pruritus, erythema and area involved) as one general measure of severity of $A D$.

As a measure of improvement of severity of $A D$ (or a component subscale) we subtracted the mean values at baseline from the mean values at the end of the follow-up period (mean improvement) in each group. In studies in which outcomes were recorded at several points in time we used the longest follow-up period during which the patients were still on randomised treatment. The publications often presented continuous data on different outcome scales. Therefore, we calculated for each study the effect sizes. If variance estimates were not given we calculated the variance according to the procedure as suggested by Follmann et al. assuming a correlation of 0.4 between the variances at baseline and at follow-up within each group. ${ }^{30}$

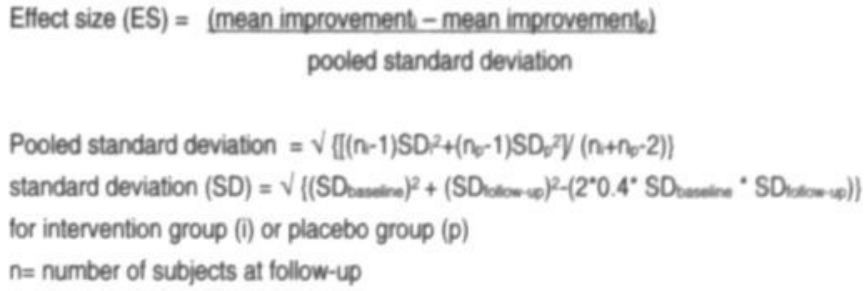


Effect sizes and its $95 \%$ confidence limits were calculated using the effect size calculator ${ }^{31}$. Because most of the studies included a relatively small number of patients, a correction procedure, using Hedges's $g$, was applied to the effect size. ${ }^{32}$

If no sufficient data on overall severity or subscales of $A D$ were given to calculate effect sizes we described the conclusions of the authors more qualitatively. When an author defined the result to be a statistically significant improvement or deterioration in the supplement group over the placebo group this was indicated with ' ++' or '- -' respectively; when the result was an improvement or deterioration for the treatment group over the placebo group but not statistically significantly so, this was indicated with ' + ' or ' - ' respectively. When the authors claimed there was no difference between the two groups this was indicated with ' 0 '.

\section{STATISTICAL ANALYSES}

To detect publication and related biases we examined funnel plot asymmetry visually and tested for publication bias using Egger's unweighted regression analysis. In this analysis the degree of funnel plot asymmetry is measured by the intercept from regression of standard normal deviates (effect sizes) against their precision (inverse of the standard error of the effect sizes). ${ }^{33}$

We pooled the summary effect sizes and corresponding confidence intervals with random effects meta-regression analysis by using the Stata statistical software package. ${ }^{34}$ The between-study variance was estimated iteratively by the empiric Bayes method. ${ }^{35}$

To explore reasons for heterogeneity of effect sizes between studies, we performed a metaregression analysis on study characteristics such as study design, population characteristics and competing interest. This analysis tests whether the effect size is modification by the study characteristics. ${ }^{36}$ Furthermore, to evaluate the influence of individual studies on the summary effect size, we repeated the pooling of effect sizes leaving out one study each time.

\section{RESULTS}

\section{SEARCH RESULTS}

An initial literature search retrieved 34 publications of clinical studies. Reference searching yielded 4 additional publications. ${ }^{37-40}$ We excluded 14 publications for the following reasons: thirteen publications addressed trials without a comparison group ${ }^{38-50}$ and one publication did not evaluate clinical outcomes of $A D$ but only of immunological outcomes. ${ }^{51}$ Of the remaining 24 publications, two pairs were more or less exact copies describing two studies, ${ }^{52-55}$ so that we ended up with 22 studies. From one abstract ${ }^{37}$ we obtained additional information from the author. 
The study characteristics are presented in table 1 . Of the 22 included studies 14 were randomized controlled trials (RCTs), 26.37.52.55-57.60.62-66.68.70 five studies were crossover trials $(\mathrm{COs})^{3-4.59 .61 .69}$ and three were clinical controlled trials (CCTs). ${ }^{58.67 .71}$ The treatment in 19 studies consisted of supplements with high GLA content, ${ }^{3.2637 .55-68}$ from the following sources; borage oil, ${ }^{55-60}$ evening primrose oil ${ }^{3-4.26 .61-69}$ and black current seed oil. ${ }^{37}$ Five studies used fish oil as treatment, ${ }^{26.52 .62 .70 .71}$ of which three ${ }^{26.48 .58}$ also had a treatment arm with GLA treatment. In the GLA trials, the patients were children in five studies, ${ }^{41.59 .64 .69-60}$ adults in 7 studies, ${ }^{37.55-56.56 .60 .63 .66}$ and a mix of children and adults in seven studies. ${ }^{3-4.26 .57 .61 .}$ 02.67 In the fish oil trials all patients were adults. Two of the seven GLA trials did not differentiate in dosages between children and adults, ${ }^{26.62}$ while the remaining five did. The daily dosage of GLA varied between 90 and $480 \mathrm{mg}$ in children and between 132 and 720 $\mathrm{mg}$ for adults, and the duration of supplementation varied between 3 to 24 weeks. Fish oil always contained both EPA and DHA varying in daily dosages between 204 and $1200 \mathrm{mg}$ for EPA, and 132 and $132 \mathrm{mg}$ for DHA, respectively, and the duration of supplementation varied between 12 and 16 weeks. Usually the placebo contained liquid paraffin, olive oil or palmkernel oil.

The three issues of most discussion between the three assessors were: the number of patients at baseline and at follow-up, and complete reporting of the outcome parameters. Unsettled issues are indicated in the footnote of table 1.

\section{QUANTTTATTVE DATA EXTRACTION}

Since variance estimates were not given, except for in one publication ${ }^{71}$ we calculated the variance according to the procedure as suggested by the Follmann method. ${ }^{30}$ In three studies that did not provide the variance for baseline and follow-up separately, we assumed the variance was equal at both times. . $^{3.60 .60}$ In three studies we had to use a ruler to measure changes in (component sub-) scales from the figures presented in the publication. ${ }^{3.63 .44}$ In two studies we pooled the results of subgroups using a fixed effects approach in order to get one effect size ${ }^{3.65}$ First, the study of Biagi et al. ${ }^{65}$ consisted of two subgroups (high and low dosages of GLA). Second, the study of Bamford $e t$ al. ${ }^{3}$ consisted of different age groups and different dosages resulting in four comparison groups: low dosage in children, high dosage in children, low dosage in adults and high dosage in adults. The study by Humphreys' $e t$ al. ${ }^{66}$ did not provide data on the improvement of AD. In order to calculate the effect size we pooled the overall scores on the component subscales surface damage, lichenification and erythema as assessed by the physician, using a fixed effects approach.

In three papers it was not clear whether the reported abbreviations SE or SEM represented standard errors or standard deviations; in those cases we chose the most conservative solutions, i.e. computing standard deviations from standard errors and number of patients in each group, ${ }^{61.70-71}$ in one publication the number of patients with complete follow-up was also not evident; in that case we also choose the most conservative estimate, i.e. the total number of patients at baseline. ${ }^{71}$ 
Table 1 Characteristics of the studies included in the meta-analysis of EFA supplementation to improve severity of atopic dermatits

\begin{tabular}{|c|c|c|c|c|}
\hline Study (year) & Design & $\begin{array}{l}\text { Range of age of } \\
\text { patients (years) }\end{array}$ & $\begin{array}{l}\text { No of patients in study at entry } \\
\text { (treatment, placebo if CCT or RCT) }\end{array}$ & $\begin{array}{l}\text { No of patients completing follow-up } \\
\text { (treatment and placebo group) }\end{array}$ \\
\hline \multicolumn{5}{|l|}{ Borage oil } \\
\hline Bahmer, 1992 & RCT & $18-42$ & $12^{\circ}$ & $12(100 \%, 100 \%)$ \\
\hline Buslau, 1996 & RCT & $18-61$ & $50(25,25)$ & $32(72 \%, 56 \%)$ \\
\hline Valsecchi, 1996 & RCT & $2-38$ & $31(15,16)$ & $28(94 \%, 87 \%)$ \\
\hline Andreassi, 1997 & CCT & $15-31$ & $\cdot$ & $60(n=30, n=30)$ \\
\hline Borrek, 1997 & $\infty$ & $3-17$ & $24(12,12)$ & $22(92 \%, 92 \%)$ \\
\hline Henz, 1999 & RCT & $14-65$ & $160(80,80)$ & $124(79 \%, 76 \%)$ \\
\hline \multicolumn{5}{|l|}{ Evening primrose oll } \\
\hline Lovell, 1981 & $\infty$ & $1.5-32$ & 32 & . \\
\hline Wright, 1982 & $\infty$ & $\begin{array}{l}0.7 \cdot 14 \\
16-60\end{array}$ & $\begin{array}{l}99,39 \text { children (20 LD, } 18 \text { HD) } \\
60 \text { adults (20 HD, } 20 \text { MD, } 20 \text { LD) }\end{array}$ & \\
\hline Bamtord, 1985 & $c 0$ & $2-66$ & 154 & 123 \\
\hline Guenther, 1987 & $\infty$ & $3-a$ & 17 & $14(82 \%)$ \\
\hline Meigel, 1987 & RCT & 7.56 & 40 "(efamol, cod-liver oil, placebo) & $34(n=16, n=11, n=7)$ \\
\hline Schalin Karrila, 1987 & RCT & $19-31$ & $25(14,11)$ & $23(93 \%, 100 \%)$ \\
\hline Bordoni, 1988 & RCT & $2-4$ & $24(12,12)$ & $24(100 \%, 100 \%)$ \\
\hline Berth Jones, 1993 & RCT & $0.58-60$ & $82(41,41)$ & $67(81 \%, 83 \%)$ \\
\hline Biagl, 1994 & ACT & $2.2-8.5$ & $51 \cdot$ & $48(n=32, n=16)$ \\
\hline Humphreys, 1994 & RCT & $16-64$ & $58(29,29)$ & $52(96 \%, 86 \%)$ \\
\hline Kiehl, 1994 & CCT & $\begin{array}{l}1-12 \\
17-48\end{array}$ & $62(39,23)$ & $\bullet$ \\
\hline Hederos, 1996 & RCT & 1.16 & $60(30,30)$ & $58(93 \%, 100 \%)$ \\
\hline \multicolumn{5}{|l|}{ Black current seed oil } \\
\hline Rilliet, 1988 & RCT & $15-78$ & $30(15,15)$ & $24(73 \%, 87 \%)$ \\
\hline \multicolumn{5}{|l|}{ Fish oils } \\
\hline Meigel, 1987 & RCT & $7-56$ & 40. (efamol, cod-liver oil, placebo) & $34(n=16, n=11, n=7)$ \\
\hline Bjomeboe, 1987, 1989 & RCT & $16-56$ & $31(16,15)$ & $23(75 \%, 73 \%)$ \\
\hline Berth Jones, 1993 & RCT & $0.6-60$ & $82(41,41)$ & $69(85 \%, 83 \%)$ \\
\hline Soyland, 1994 & RCT & $18-64$ & $145 *$ & $120(n=57, n=63)$ \\
\hline Gimenez Amau, 1997 & CCT & $\begin{array}{l}\text { mean } 24.2 \\
\text { SD } 6.8\end{array}$ & 48. & - \\
\hline \multicolumn{5}{|c|}{$\begin{array}{l}\mathrm{RCT}=\text { randomized controlled trial } \\
\mathrm{CCT}=\text { clinical controlled trial } \\
\mathrm{CO}=\text { Cross over trial; } \\
\mathrm{HD}=\text { high dose; } \mathrm{MD}=\text { middle dose; } L \mathrm{D}=\text { low dose } \\
\text { " not clearly described in publication }\end{array}$} \\
\hline
\end{tabular}




\begin{tabular}{ll}
\hline Total amount of active EFA per day (brand name it mentioned) placebo & $\begin{array}{l}\text { Duration of } \\
\text { supplementation (weeks) interest (grade) }\end{array}$
\end{tabular}

$480 \mathrm{mg}$ GLA (Glando)
$480 \mathrm{mg}$ GLA (Glando)

aduls: $400 \mathrm{mg}$ GLA ; intants; $240 \mathrm{mg}$ GLA

$132 \mathrm{mg}$ GLA

$360 \mathrm{mg}$ GLA (Glandol)

$690 \mathrm{mg}$ GLA

adults $360 \mathrm{mg}$ GLA (Etamol)

children $180 \mathrm{mg}$ GLA(Etamol)

children (Elamol): $90 \mathrm{mg}$ GLA (LD), $180 \mathrm{mg}$ GLA (HD)

adults (Elamol): $80 \mathrm{mg}$ GLA (LD), $360 \mathrm{mg}$ GLA (MD ) $540 \mathrm{mg}$

GLA (HD)

$600 \mathrm{mg}$ GLA

$3060 \mathrm{mg}$ EPA, $1920 \mathrm{mg}$ DHA

$2000 \mathrm{mg}$ EPA, $1300 \mathrm{mg}$ DHA children (Elamol); $180 \mathrm{mg}$ GLA (LD), $450 \mathrm{mg}$ GLA (HD)

adults (Etamo); $540 \mathrm{mg}$ GLA (LD), $720 \mathrm{mg}$ GLA (HD)

3-7 years 4 capsules Etamol, 7-14 years 6 capsules, $>14$ yrs 8 capsules

total 4.68 gram of LA and GLA (Etamol)

$360 \mathrm{mg}$ GLA

$267 \mathrm{mg}$ GLA (Etamol)

$480 \mathrm{mg}$ GLA (Epogam)

depending on weight intant :

HO 43.3 mgkg/day; LD 21.7 mg/kg/day

6 gram Epogam

intants; first 4 weeks $480 \mathrm{mg}$ GLA than $360 \mathrm{mg}$ GLA for 8 weeks' adults (Epogam); first 10 days $480 \mathrm{mg}$ than 10 weeks $240 \mathrm{mg}$

1.12 years $320 \mathrm{mg}$ GLA (Epogam)

$12-16$ years $480 \mathrm{mg}$ GLA (Epogam)

grape seed oil

\section{Fish oils}

$1800 \mathrm{mg}$ EPA, $1200 \mathrm{mg}$ DHA

413 mg GLA , 204 mg EPA, 132 mg DHA (Etamol Marine)
13

olive oil

olive oil

adults: liquid paraffin

children olive oil

$500 \mathrm{mg}$ paimkernel ol

$500 \mathrm{mg}$ paimkemel ofl

lquid parattin

maize seed oll

liquid paraffin

3

3

liquid paraftin

12

2

olve oil

liquid paraftin

olive oll.

adults: liquid paraffin

children olive oll

olive oil

liquid paraffin

16

12

16

2

corn oil

oleic acid

12

2

12

0

12

0

12

4

16

8

0

2

0

0 
Table 2 Effect of EFA supplementation on overall severity of atopic dermatitis, trials ranked by weight"

\begin{tabular}{|c|c|c|c|c|c|c|c|c|}
\hline \multirow[t]{2}{*}{ Study (year) } & & \multicolumn{2}{|c|}{ Treatment group } & \multicolumn{2}{|l|}{ Placebo group } & \multirow{2}{*}{$\begin{array}{l}\text { EHect size } \\
\text { (Hedges) }\end{array}$} & \multirow{2}{*}{$\begin{array}{l}95 \% \text { Confidence } \\
\text { limits for effect size }\end{array}$} & \multirow{2}{*}{$\begin{array}{l}\text { Weight } \\
\text { (1/SE) }\end{array}$} \\
\hline & & $\begin{array}{l}\text { mean } \\
\text { improvement }\end{array}$ & n & $\begin{array}{l}\text { mean } \\
\text { improvement }\end{array}$ & $n$ & & & \\
\hline \multicolumn{9}{|l|}{ GLA } \\
\hline \multicolumn{9}{|l|}{ Borage oil } \\
\hline Henz, 1999 & COSTA-score & 12.00 & 79 & 11.00 & 78 & 0.20 & $-0.11,0.51$ & 6.25 \\
\hline Borrek, 1997 & COSTA-score & 5.00 & 22 & 9.00 & 22 & -0.25 & $-0.84,0.35$ & 3.30 \\
\hline Valsecchi, 1996 & Atherton-score & 70.85 & 12 & 48.53 & 15 & 0.53 & $-0.24,1.30$ & 2.54 \\
\hline Bahmer, 1992 & ADASI & & & & & + & & \\
\hline Buslau, 1996 & ADASI & & & & & + & & \\
\hline pooled effect size & & & & & & 0.14 & $-0.21,0.49$ & \\
\hline \multicolumn{9}{|l|}{ Evening primrose oll } \\
\hline Bamtord, 1985 & $\begin{array}{l}\text { pooled effects over age } \\
\text { groups and dosages }\end{array}$ & 0.96 & $\begin{array}{c}12 \\
3\end{array}$ & 0.94 & 123 & 0.04 & $-0.21,0.29$ & 7.84 \\
\hline Humphreys, 1994 & $\begin{array}{l}\text { pooled effects of component } \\
\text { subscales }\end{array}$ & & & & & 0.18 & $-0.20,0.57$ & 5.00 \\
\hline Berth Jones, 1993 & Leicester score & 8.48 & 33 & 7.15 & 34 & 0.08 & $-0.40,0.56$ & 4.13 \\
\hline Lovell, 1981 & clinical severity & 0.99 & 32 & 0.62 & 32 & 0.19 & $-0.30,0.68$ & 3.99 \\
\hline Hederos, 1996 & global assessment & 11.0 & 30 & 13.8 & 30 & -0.17 & $-0.76,0.25$ & 3.87 \\
\hline Schalin Karrila, 1987 & overall severity & 20.00 & 13 & 12.50 & 11 & 0.35 & $-0.46,1.16$ & 2.42 \\
\hline Bordoni. 1988 & clinical score atopic dermatitis & 7.59 & 12 & 0.40 & 12 & 1.43 & $0.53,2.34$ & 2.17 \\
\hline Biagi, 1994 & $\begin{array}{l}\text { overall severity, pooled over } \\
\text { dosages }\end{array}$ & & & & & 0.54 & $-0.99,2.06$ & 1.28 \\
\hline Wright, 1982 & overall severity & & & & & t+ & & \\
\hline Guenther, 1987 & severity & & & & & 0 & & \\
\hline Meigel, 1987 & clinical improvement & & & & & + & & \\
\hline Kiehl, 1994 & ADASI & & & & & 0 & & \\
\hline pooled effect size & & & & & & 0.17 & $-0.06,0.39$ & \\
\hline Overall pooled effect siz & ze of GLA supplementation & & & & & 0.15 & $-0.02,0.33$ & \\
\hline \multicolumn{9}{|l|}{ Fish oils } \\
\hline Soyland, 1994 & mean clinical score & 1.3 & 58 & 1.0 & 63 & 0.12 & $-0.24,0.48$ & 5.49 \\
\hline Berth Jones, 1993 & Leicester score & 2.54 & 35 & 7.15 & 34 & -0.27 & $-0.74,0.21$ & 4.09 \\
\hline Gimenez Amau, 1997 & Rajka score & 5.3 & 16 & 8.8 & 16 & -0.48 & $-1.19,0.22$ & 2.79 \\
\hline Bjorneboe, 1987, 1989 & severity & 1.8 & 8 & 1.5 & 7 & 0.10 & $-0.91,1.12$ & 1.93 \\
\hline \multicolumn{6}{|c|}{ pooled effect size of fish oil supplementation } & -0.06 & $-0.32,0.21$ & \\
\hline
\end{tabular}

- Weight is defined as $1 /$ standard error

++ data not fully presented, however, authors claim statistical significant improvement in supplement group over placebo group

+ data fully presented, however, authors claim statistical non-significant improvement in supplement group over placebo group

0 data fully presented, however authors claim no differences between placebo and supplement group 


\section{OVERALL SEVERITY OF AD}

Pooled effects sizes are presented in table 2. The pooled effect size for GLA, calculated from 11 trials, is $0.15(\mathrm{CL}-0.02 ; 0.32, P=0.09)$. In eight publications ${ }^{4.37 .55-56.58 .62 .67 .69}$ no sufficient data on overall severity or component subscales of $A D$ were given to calculate effect sizes. Two of them claimed statistically significantly improvements in the treatment group, ${ }^{4.56}$ two claimed a positive non-significant improvement in the treatment group, ${ }^{55.62}$ and two claimed no differences in improvements in the treatment group, ${ }^{67.69}$ always compared to the placebo group (Table 2). In two publications no conclusion was given at all. ${ }^{37.58}$. The pooled effect size calculated from the four fish oil trials was $-0.06(95 \% \mathrm{CL}-0.32 ; 0.21)$.

\section{COMPONENT SUBSCALES OF AD}

Tables 3 and 4 summarize the component subscales as scored by the physician and the patients, respectively. None of the component subscales (i.e. area, pruritus, erythema, lichenification, dryness or scaling) showed a statistically significantly greater improvement in the treatment group over the placebo group after supplementation with either GLA or fish oil. As an exception, scaling as scored by the physician improved significantly in the fish oil treatment group compared to the placebo group (Table 3). The qualitative conclusions were as heterogeneous as the quantitative results (Table 3 ).

\section{PUBLICATION BIAS}

The number of studies was insufficient to check for publication or related bias except for studies of GLA supplementation, with severity of AD or itch as the outcome. The funnel plot (Figure 1) gives a slight hint of publication bias since among the studies with low precision (high SE) positive studies were over-represented, compared to studies with high precision (low SE) (figure). This was not confirmed by the Egger's test $(P=0.15)$.

Leaving out one study at the time did not change the pooled effect size of GLA much: the pooled effect size varied from 0.13 to 0.19 , the confidence intervals varied between -0.05 and 0.40 .

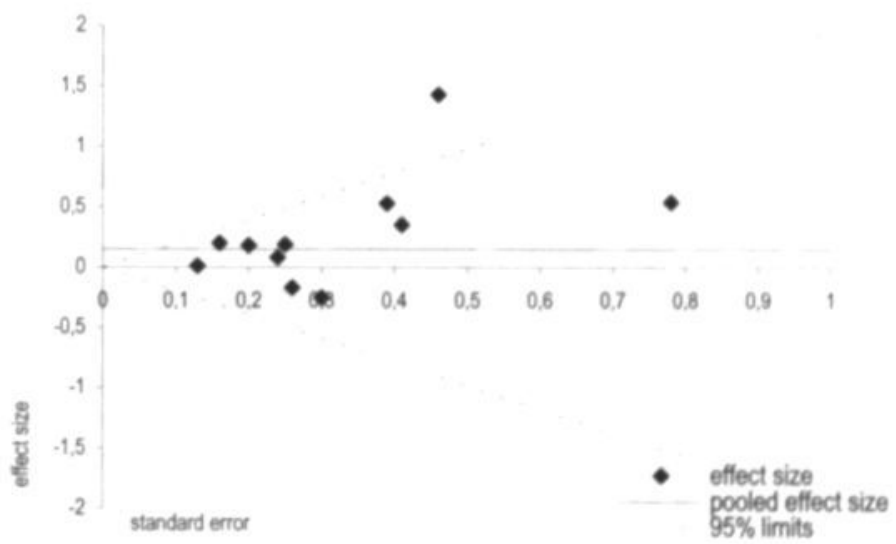

Figure 1. Funnelplot of 11 GLA supplementation trials, effect size for severity of atopic dermatitis plotted against standard error 
Table 3 Effect of EFA supplementation assessed by physician on component subscale outcomes, effect sizes (standard error)

\begin{tabular}{|c|c|c|c|c|c|c|c|}
\hline Study (year) & area & pruntus & erythema & scaling & Icthenification & dryness & other \\
\hline \multicolumn{8}{|l|}{ GLA } \\
\hline \multicolumn{8}{|l|}{ Borage oll } \\
\hline Buslau, 1996 & $\rightarrow$ & & & & & & 1 \\
\hline Valeecchi, 1996 & & & & & & & 2 \\
\hline Andreassi, 1997 & & $H$ & $\rightarrow$ & 0 & & & 3 \\
\hline Borrek, 1997 & & $-1.30(0.33)$ & & & & & 4 \\
\hline Henz, 1999 & & & + & & + & & 5 \\
\hline pooled effect size (95\% C) & & & & & & & \\
\hline \multicolumn{8}{|l|}{ Evening primrose of } \\
\hline Wright, 1962 & & $\leftrightarrow$ & 0 & + & & & \\
\hline Bamtord, 1985 & 0 & & 0 & 0 & 0 & & 6 \\
\hline Meigel, 1987 & + & 0 & & & & & 7 \\
\hline Schalin Karrla, 1987 & $0.15(0.41)$ & & & & & $0.55(0.42)$ & 8 \\
\hline Guenther, 1997 & & & & & & & 9 \\
\hline Berth Jones, 1993 & $0.29(0.25)$ & & $\leftrightarrow$ & & 0 & 0 & 10 \\
\hline Blag. 1994 & $0.36(1.00)$ & & & & & & \\
\hline Humphreys. 1994 & & & $0.37(0.28)$ & & $-0.21(0.28)$ & & 11 \\
\hline Hederos, 1996 & $0.23(0.26)$ & $-0.15(0.26)$ & $-0.09(0.26)$ & $0.04(0.26)$ & $-0.22(0.26)$ & $0.24(0.26)$ & 12 \\
\hline pooled effect size (95\% CL) & $0.25(-0.07,0.57)$ & & $0.08(-0.29,0.46)$ & $-0.04(-0.55,0.47)$ & $-0.22(-0.59,0.16)$ & $0.09(-0.68,0.85)$ & \\
\hline \multicolumn{8}{|l|}{ Black current seed ofl } \\
\hline Rilliet, 1968 & 0 & & & & & & 13 \\
\hline $\begin{array}{l}\text { Overall pooled effect size GLA } \\
\text { (95: CL) }\end{array}$ & $0.25(-0.08,0.57)$ & $.0 .71(-1.83,0.42)$ & $0.06(-0.29,0.46)$ & $-0.04(-0.55,0.47)$ & $-0.22(-0.59,0.16)$ & $0.09(-0.68,0.85)$ & \\
\hline \multicolumn{8}{|l|}{ Fish olls } \\
\hline Meigel, 1987 & + & & & & & & \\
\hline Bipmeboe, 1987,1989 & $0.07(0.42)$ & & $0.00(0.52)$ & $0.15(0.52)$ & $0.22(0.52)$ & & 14 \\
\hline Berth Jones, 1993 & $0.15(0.14)$ & 0 & & & & & \\
\hline Soyland, 1994 & $0.12(0.19)$ & $0.43(0.18)$ & $.0 .25(0.18)$ & $0.39(0.18)$ & $0.31(0.18)$ & & 15 \\
\hline Gimenez Amav, 1997 & $-0.51(0.36)$ & & & & & & 16 \\
\hline $\begin{array}{l}\text { pooled effect size of fish olls } \\
\text { (95\% C4) }\end{array}$ & $0.02(-0.26,0.22)$ & & $.0 .22(-0.56,0.11)$ & $0.36(0.03,0.70)$ & $0.30(-0.08,0.63)$ & & \\
\hline
\end{tabular}

$\mathrm{CL}=95 \%$ confidence limits

++ data not fully presented, however, authors claim statistical significant decrease in complaints in supplement group over placebo group

+ data not fully presented, however, authors claim statistical non-significant decrease in complaints in supplement group over placebo group

0 data fully presented, however authors claim no differences between placebo and supplement group

Effect sizes (ES) for other outcomes

1 total $\lg E 0$

2 total $\lg E 0$

3 vesiculation ++ , oozing ++ , less use drugs in treatment group compared to placebo group

4 acute symptoms (erythema, oedema, papules, crusts, excoriation) 0 , chronic symptoms (scale, lichenification, (de)-pigmentation 0

5 oozing ++, vesiculation + , crusting + , excoriation + , insomnia + , corticosteroid use ES -0.98 (SE 0.17 )

6 excoriation 0 , weeping 0

7 use topical steroids 0

8 overall respons ES -0.15 (SE 0.41), inflammation ES 0.29 (SE 0.41), use emollients ES 0.13 (SE 0.41), use topical steroids ES 3.29 (SE 0.64)

9 fidget +

10 cracking ++, excoriation 0 , largest reduction in steroid use in placebo group

11 surface damage ES 0.39 (SE 0.28)

12 crusting ES 0.00 (SE 0.26), fidget ES -0.35 (SE 0.26), overall impression ES 0.25 (SE 0.26)

13 use topical steroids 0 , lesions 0 , scerosis 0

14 visibility ES 0.25 (SE 0.52), excoriation ES - 0.13 (SE 0.52), weeping ES 0.04 (SE 0.52)

15 induration ES 0.00 (SE 0.18)

16 Rajka score ES -0.48 (SE 0.36), nule of nine ES -0.51 (SE 0.36) 
Table 4. Effect of EFA supplementation assessed by patient on component subscale outcomes, effect sizes (standard error)

\begin{tabular}{|c|c|c|c|c|c|}
\hline Study (year) & pruritus & enthema & scaling & dryness & other \\
\hline \multicolumn{6}{|l|}{ GLA } \\
\hline \multicolumn{6}{|l|}{ Borage oil } \\
\hline Buslau, 1996 & $\omega$ & & & & 1 \\
\hline Andreassi. 1997 & + & & & & \\
\hline Borrek & & & & & 2 \\
\hline Henz, 1999 & 0 & & & & 3 \\
\hline \multicolumn{6}{|l|}{ Evening primrose of } \\
\hline Lovel, 1981 & & & & & 4 \\
\hline Wright, 1982 & 0 & 0 & 0 & & \\
\hline Bamtord, 1985 & 0 & 0 & & & 5 \\
\hline \multicolumn{6}{|l|}{ Mengel, 1987} \\
\hline Schalin Karrila, 1987 & $0.70(0.42)$ & & & & \\
\hline Berth Jones, 1993 & 0 & & & & \\
\hline Biag. 1994 & $0.23(0.63)$ & & & & \\
\hline Humphreys, 1994 & $0.23(0.28)$ & $0.28(0.28)$ & $0.20(0.28)$ & $0.29(0.28)$ & 6 \\
\hline Hederos, 1996 & $-0.05(0.26)$ & $0.29(0.26)$ & $0.06(0.26)$ & $-0.07(0.26)$ & 7 \\
\hline Overal pooled effect size of GLA ( $96 \%$ CL) & $0.19(-0.14 ; 0.52)$ & $0.29(-0.09,0.66)$ & $0.14(-0.24 ; 0.51)$ & $0.10(-0.28: 0.47)$ & \\
\hline \multicolumn{6}{|l|}{ Fish olls } \\
\hline Bipmeboe, 1987, 1989 & $0.92(0.55)$ & $0.43(0.52)$ & $0.73(0.54)$ & & 8 \\
\hline Soyland, 1994 & $0.05(0.19)$ & $0.00(0.19)$ & $0.17(0.19)$ & & 9 \\
\hline Overall pooled effect size of EPA $(95 \%$ CL) & $0.33(-0.47,1.13)$ & $0.05(-0.30,0.40)$ & $0.23(-0.12,0.58)$ & & \\
\hline \multicolumn{6}{|c|}{$\begin{array}{l}++ \text { data not fully presented, however, authors claim statistical significant decrease in complaints in supplement group over placebo group } \\
+ \text { data not fully presented, however, authors claim statistical non-significant decrease in complaints in supplement group over placebo } \\
\text { group }\end{array}$} \\
\hline 0 data not fully presented, however authors & Laim no diflerences b & tween placebo and $s$ & upplement group & & \\
\hline \multicolumn{6}{|l|}{ Eflect sizes (ES) for other outcomes } \\
\hline \multicolumn{6}{|l|}{ less use emolients in treatment group } \\
\hline \multicolumn{6}{|l|}{2 subjective severity ES -0.54 (SE 0.31 ) } \\
\hline \multicolumn{6}{|l|}{3 insomnia + } \\
\hline \multicolumn{6}{|c|}{4 patient's assessment clinical severity ES 0.76 (SE 0.54) } \\
\hline \multicolumn{6}{|c|}{5 pruritus 0 , discomfort with appearance 0} \\
\hline \multicolumn{6}{|l|}{6 overall impression ES 0.29 (SE 0.28) } \\
\hline \multicolumn{6}{|l|}{$\begin{array}{l}7 \text { crusting ES } 0.21 \text { (SE 0.26), sleep ES - } \\
\text { assessment ES } 0.14 \text { (SE 0.26) }\end{array}$} \\
\hline \multicolumn{6}{|c|}{$\begin{array}{l}8 \text { effect on daily life ES } 0.42 \text { (SE 0.52), visibility ES } 0.75 \text { (SE 0.54), patient's assessment overall improvement ES } 0.76 \text { (SE } 0.54 \text { ) } \\
9 \text { mean clinical score patient ES }-0.05 \text { (SE 0.19), area ES - } 0.17 \text { (SE 0 19), effect on dally ife ES } 0.13 \text { (SE } 0.19 \text { ) }\end{array}$} \\
\hline 9 mean clinical score patient ES - -0.05 (SE 0 & 19), area ES -0.17 (S & E 0.19), effect on dall & $y$ life ES 0.13 (SE 0.1 & & \\
\hline
\end{tabular}

\section{SENSITIVITY ANALYSIS}

We further examined the role of competing interest, age of the patients, duration of supplementation and the source of GLA to explore their influence on the effect sizes for severity of $A D$ using a meta-regression analysis. We divided the markers of competing interest into two groups: 5 trials with grade 0 or 1 vs 6 trials with grade 2 or 3 . The analysis did not give evidence that there was an influence of the competing interest on the effect size $(P=0.21)$. Furthermore, age of the subjects (age 0-12 (3 trials) vs 12- 64 (4 trials)) did not influence the effect size $(P=0.44)$, nor did the source of GLA ( 3 trials with borage oil vs 8 trials with evening primrose oil) $(P=0.83)$ or duration of supplementation $(P=0.33)$. For fish oil supplementation we could not perform this analysis because of lack of sufficient number of studies. 


\section{DISCUSSION}

In this meta-analysis we could not confirm that supplementation of GLA exerts a large effect on the severity of $A D$. If we excluded one trial at the time, the results did not change substantially, i.e. the effect size did not change much and neither did the $95 \%$ confidence limits (data not shown). When we translate the effect size into COSTA-score values to facilitate clinical interpretation, the change in clinical score appears to be rather small. COSTA-scores can vary between 0-100 points. Assuming an average SD of about 10 (based on (59) and (60)), the effect size calculated in this meta-analysis, 0.15 (CL -0.02 , $0.33)$ would have resulted in a decrease of $1.5(\mathrm{CL}-0.20,3.3)$ points on the COSTA-score. We consider this as a clinically non-significant improvement. The effect of fish oil was certainly smaller.

Our meta-analysis has a low power to detect a moderate effect of supplementation with GLA in AD. First, most trials had a low number of patients. Nine of the 22 trials included less than 20 subjects per group so that randomization was not likely to ensure the desired prognostic comparability between treatment and placebo groups. This justified a random-effect model for the statistical pooling of the effects, which leads to wider confidence limits around the effect estimate, compared to a fixed effects model. Second, we made conservative assumptions when the publications did not provide sufficient detail to calculate effect sizes. Also, in cross-over trials the within-subject variability was not efficiently accounted for. Third, the cross-over trials lacked a wash-out period so that a treatment effect may have been underestimated by carry-over to the placebo period. Therefore, we cannot exclude a true small effect of GLA supplementation.

The former meta-analysis from $1989^{22}$ and a more recent review ${ }^{21}$ came to the conclusion that GLA had a beneficial effect on the severity of AD. We believe that this meta-analysis is more substantiated than the former meta-analysis for the following reasons.

\section{Selective publication:}

The former meta-analysis, however, used a selective inclusion of trials, leaving out one of the major published trials (by Bamford et al.) and came to the conclusion that GLA had a large effect on the severity of AD. When we left out trials one by one from our pooling (including the Bamford study), the results did not change substantially. We have found no evidence of publication bias or an influence of competing interest of the authors of the trials.

\section{Selective reporting within trials:}

We were unable to calculate effect sizes in 8 out of 22 trials $(36 \%)$. Because we did not want to lose the information from these studies, we have described their qualitative conclusions in the tables. These conclusions were in line with the quantitative result (pooled effect size) and the heterogeneity between studies.

Not all trials reported changes in component subscales of atopic dermatitis, even though in most trials these component subscales were measured. If the results of the component subscales had been presented in the publications, it would have created the opportunity to combine more trials to get a more accurate result. Nevertheless, we used the maximum of information that could be obtained from the trials and included as many trials as possible, like for instance the study by Guenther, ${ }^{69}$ a letter to the editor of a journal. GLA supplementation showed no benefit on any of the component subscale measures such as 
itch, scaling and lichenification. The only exception was the statistically significant result of fish oil supplementation on scaling, but because of the many comparisons, we think that this was a chance finding. In the previous meta-analysis and review ${ }^{21-22}$ itch was reported to be significantly reduced as result of GLA supplementation, but it is not clear on how many comparisons and studies this was based, so that this could have been a chance finding too. Our meta-analysis agrees with cautious comments and reviews. ${ }^{20.24-25}$ These suggested that in order to be able to give a sustained conclusion, trials should be more punctual in presenting baseline values and complete reporting of the overall severity of AD or complete results on component sub-scales.

\section{Influence of study cbaracteristics:}

In the sensitivity analysis we evaluated whether study characteristics had an influence on the effect size. The influence of dosage could not be tested because most trials used comparable dosages. We were unable to identify an influence of age, but the power to detect such influence is low because of the limited number of trials. From a biological point of view especially infants should benefit most from GLA supplementation. Galli $\theta t$ al. ${ }^{20}$ has shown in the early 1990s that changes in n-6 EFAs precede the development of AD. Furthermore, n-6 EFA profiles of breast milk are different between mothers of infants that later developed eczema compared to mothers of infants that remain unaffected. ${ }^{6.72}$ In a preventive trial (Van Gool, submitted for publication) we could not show that GLA had a preventive effect on $A D$ in an intention-to-treat analysis, but the infants who had the highest increase in plasma phospholipid GLA level developed the least severe AD at the age of one year.

We conclude that EFA supplementation is of no proven effectiveness for the treatment of AD. Effectiveness in certain subgroups, such as young children or patients with severe AD, may be present, but the published studies do not present evidence to substantiate or refute this.

\section{REFERENCES}

1. Worldwide variation in prevalence of symptoms of asthma, allergic minoconjunctivitis, and atopic eczema: ISAAC. The International Study of Asthma and Allergies in Childhood (ISAAC) Steering Committee. Lancet. 1998;351:1225-32.

2. Burr ML. Epidemiology of Clinical Allergy. Basel: Karger, 1993.

3. Bamford JT, Gibson RW, Renier CM. Atopic eczema unresponsive to evening primrose oil (linoleic and gamma-linolenic acids). J Am Acad Dermatol. 1985;13:959-65.

4. Wright S, Burton JL. Oral evening-primrose-seed oil improves atopic eczema. Lancet. 1982:2:1120-2.

5. Brown WR, Hansen AE. Arachidonic and Linolic Acid of the serum in normal and eczematous human subjects. Proc Soc Exp Biol Med. 1937;36:113-117.

6. Businco L, loppi M, Morse NL, et al. Breast milk from mothers of children with newly developed atopic eczema has low levels of long chain polyunsaturated fatty acids. J Allergy Clin Immunol. 1993:91:1134-9.

7. Lindskov R, Holmer G. Polyunsaturated fatty acids in plasma, red blood cells and mononuclear cell phospholipids of patients with atopic dermatitis. Allergy. 1992;47:517-21.

8. Thijs C, Houwelingen A, Poorterman I, et al. Essential fatty acids in breast milk of atopic mothers: comparison with non-atopic mothers, and effect of borage oil supplementation. Eur $\mathrm{J}$ Clin Nutr. 2000;54:234-8. 
9. Wright S, Sanders TA. Adipose tissue essential fatty acid composition in patients with atopic eczema. Eur J Clin Nutr. 1991;45:501-5.

10. Wright S. Atopic dermatitis and essential fatty acids: a biochemical basis for atopy? Acta Derm Venereol.Suppl. Suppl. 1985;114:143-5.

11. Manku MS, Horrobin DF, Morse N, et al. Reduced levels of prostaglandin precursors in the blood of atopic patients: defective delta-6-desaturase function as a biochemical basis for atopy. Prostaglandins Leukot Med. 1982;9:615-28.

12. Horrobin DF. Nutritional and medical importance of gamma-linolenic acid. Prog Lipid Res. 1992:31:163-94.

13. Melnik B, Plewig G. Are disturbances of omega-6-fatty acid metabolism involved in the pathogenesis of atopic dermatitis? Acta Derm Venereol.Suppl. 1992;176:77-85.

14. Ziboh VA. Prostaglandins, leukotrienes, and hydroxy fatty acids in epidermis. Semin Dermatol. 1992:11:114-20.

15. Ziboh VA, Fletcher MP. Dose-response effects of dietary gamma-linolenic acid-enriched oils on human polymorphonuclear-neutrophil biosynthesis of leukotriene B4. Am J Clin Nutr. 1992;55:3945.

16. Ziboh VA, Miller CC, Cho Y. Metabolism of polyunsaturated fatty acids by skin epidermal enzymes: generation of antiinflammatory and antiproliferative metabolites. Am J Clin Nutr 2000:71:361s-6s.

17. Bangha $E$, Amon U. Gamma linolenic acid for atopic dermatitis. A review. Aktuelle Dermatologie 1999:25:8-9.

18. Ring J, Kleinheinz A. Nachtkerzenol bei Neurodermitis? Medizinische Monatsschrift fur Pharmazeuten 1991:14:282.

19. Melnik B. Prophylaxis and therapy of atopic eczema with gammalinolenic acid. Allergologie. 1993;16:454-457.

20. Tronnier $\mathrm{H}$, Heinrich $\mathrm{U}$, Aygen $\mathrm{S}$. Treatment of atopic dermatitis with unsaturated fatty acids. $\mathrm{H}+\mathrm{G}$ Z Hautkr. 1993;68:562-572.

21. Horrobin DF. Essential fatty acid metabolism and its modification in atopic eczema. Am J Clin Nutr 2000:71:367s-372s.

22. Morse PF, Horrobin DF, Manku MS, et al. Meta-analysis of placebo-controlled studies of the efficacy of Epogam in the treatment of atopic eczema. Relationship between plasma essential fatty acid changes and clinical response. Br J Dermatol. 1989;121:75-90.

23. Sharpe GR, Farr PM. Evening primrose oil and eczema. Lancet. 1990;335.

24. Gamolenic acid in atopic eczema: Epogam. Drug Ther Bull. 1990;28:69-70.

25. Hoare C, Li Wan Po A, Williams H. Systematic review of treatments for atopic eczema. Health Technol Assess. 2000;4:1-191.

26. Berth Jones J, Graham Brown RAC. Placebo-controlled trial of essential fatty acid supplementation in atopic dermatitis. Lancet. 1993;341:1557-1560.

27. Horrobin DF, Morse PF. Evening primrose oil and atopic eczema. Lancet. 1995;345:260-1.

28. Galli E, Picardo M, Chini L, et al. Analysis of polyunsaturated fatty acids in newborn sera: A screening tool for atopic disease? Br J Dermatol. 1994;130:752-756.

29. Vandenbroucke JP. Passive smoking and lung cancer: a publication bias? BMJ. (Clin Res Ed) 1988:296:391-2.

30. Follmann D, Elliott P, Suh I, Cutler J. Variance imputation for overviews of clinical trials with continuous response. J Clin Epidemiol. 1992;45:769-73.

31. Coe R. What is an 'Effect Size'?, cem.dur.ac.uk/ebeuk/research/effectsize/ESCalcGuide.doc, 2000.

32. H. Cooper LVH. The Handbook of Research Synthesis. New York: Russell Sage Foundation, 1994.

33. Egger M, Davey Smith G, Schneider M, et al. Bias in meta-analysis detected by a simple, graphical test. BMJ. 1997;315:629-34.

34. StataCorp. Stata Statistical Software: Release 7.0. . 7 ed. Texas: College Station, 2001.

35. Berkey CS, Hoaglin DC, Mosteller F, et al. A random-effects regression model for meta-analysis. Stat Med. 1995;14:395-411.

36. Thompson SG, Sharp SJ. Explaining heterogeneity in meta-analysis: a comparison of methods. Stat Med. 1999;18:2693-708.

37. Rilliet A, Queille C. Saurat J-H. Effects of gamma-linolenic acid in atopic dermatitis. Dermatologica 1988;177:257. 
38. Shimasaki H. Pufa content and effect of dietary intake of gamma-linolenic acid-rich oil on profiles of $n-6, n-3$ metabolites in plasma of children with atopic eczema. J Nutr Biochem. 1995;19:183192.

39. Stewart JCM, Morse PF, Moss M, et al. Treatment of severe and moderately severe atopic dermatitis with evening primrose oil (Epogam): a multicenter study. J Nutr Med. 1991;2:9-15.

40. Nissen HP, Wehrmann W, Kroll U, et al. Veranderungen im plasma-lipid-muster bei patienten mit neurodermitis. Fat Science and Technology. 1988;7:268-271.

41. Biagi PL, Bordoni A, Masi M, et al. A long-term study on the use of evening primrose oil (Efamol) in atopic children. Drugs Exp Clin Res. 1988;14:285-90.

42. Hollmann J, Michelsen S, Jansen T, et al. Effect of high-dose oral therapy with evening primrose oil on the main lipid fractions in severe atopic dermatitis. Z Hautkr. 1996;71:115-120.

43. Skogh M. Atopic eczema unresponsive to evening primrose oil (linoleic and gamma-linolenic acids). J Am Acad Dermatol. 1986;15:114-5.

44. Wehrmann W, Niedecken H, Bauer R. Klinische und immunmodulatorische Effekte einer Behandlung mit ungesattigten Fettsauren bei atopischer Dermatitis. 1987;62 Suppl 1:111-5.

45. Landi G. Oral administration of borago oil in atopic dermatitis. Journal of Applied Cosmetology. 1993;11:115-120.

46. Patrizi A, Neri I, Orlandi C, et al. Efficacy evaluation of Oenothera biennis oil in 57 patients with atopic dermatitis. Giomale Italiano di Dermatologia e Venereologia. 1994;129:Lix-Lxiii.

47. Fiocchi A, Sala M, Signoroni P, et al. The efficacy and safety of gamma linolenic acid in the treatment of infantile atopic dermatitis. J Int Med Res. 1994;22:24-32.

48. Melnik BC, Bahmer FA. Treatment of atopic dermatitis with Glandol registered and Epoleum registered - A comparative study. Aktuelle Dermatologie. 1995;21:215-219.

49. Furukawa F, Tokura Y, Takigawa M, et al. Clinical effect of gamma-linolenic acid supplement food on atopic dermatitis. Skin Res. 1998;20:73-78.

50. Schafer L, Kragballe K. Supplementation with evening primrose oil in atopic dermatitis: Effect on fatty acids in neutrophils and epidermis. Lipids. 1991;26:557-560.

51. Lehmann B, Hubner C, Jacobi $\mathrm{H}$, et al. Effects of dietary gamma -linolenic acid-enriched evening primrose seed oil on the 5-lipoxygenase pathway of neutrophil leukocytes in patients with atopic dermatitis. J Dermatolog Treat. 1995;6:211-218.

52. Bjomeboe A, Soyland E, Bjomeboe GE, Rajka G, Drevon CA. Effect of n-3 fatty acid supplement to patients with atopic dermatitis. J Intern Med. Supplement. 1989;225:233-6.

53. Bahmer FA, Schaefer J. Die Wirkung von Borretschoel auf die atopic dermatitis - eine Zeitreiheanalytische Studie. Dermatologie. 1992;18:85-8.

54. Bjomeboe A, Soyland E, Bjomeboe GE, et al. Effect of dietary supplementation with eicosapentaenoic acid in the treatment of atopic dermatitis. Br J Dermatol. 1987;117:463-9.

55. Bahmer FA, Scafer J. Treatment of atopic dermatitis with borage oil (Glandol) - A time series analysis study. Kinderarztl Prax. 1992;60:199-202.

56. Buslau M, Thaci D. Atopic dermatitis: Borage oil for systemic therapy. Zeitschrift fur Dermatologie. 1996;182:132+134-136.

57. Valsecchi R, Di Landro A, Pansera B, et al. Gammalinolenic acid in the treatment of atopic dermatitis. J Eur Acad Dermatol Venereol. 1996;7:77-79.

58. Andreassi M, Forleo P, Di Lorio A, et al. Efficacy of gamma -linolenic acid in the treatment of patients with atopic dermatitis. J Int Med Res. 1997;25:266-274.

59. Borrek S, Hildebrandt A, Forster J. Borage seed-oil and atopic dermatitis. Klin Padiatr. 1997;209:100-104.

60. Henz BM, Jablonska S, Van De Kerkhof PCM, et al. Double-blind, multicentre analysis of the efficacy of borage oil in patients with atopic eczema. Br J Dermatol. 1999;140:685-688.

61. Lovell CR, Burton JL, Horrobin DF. Treatment of atopic eczema with evening primrose oil. Lancet. 1981;1:278.

62. Meigel W, Dettke T, Meigel EM, et al. Additional oral treatment of atopical dermatitis with unsaturated fatty acids. Z Hautkr. 1987;62:100-103.

63. Schalin Karrila M, Mattila L, Jansen CT, et al. Evening primrose oil in the treatment of atopic eczema: effect on clinical status, plasma phospholipid fatty acids and circulating blood prostaglandins. Br J Dermatol. 1987;117:11-9.

64. Bordoni A, Biagi PL, Masi M, et al. Evening primrose oil (Efamol) in the treatment of children with atopic eczema. Drugs Exp Clin Res. 1988;14:291-7. 
65. Biagi PL, Bordoni A, Hrelia S, et al. The effect of gamma-linolenic acid on clinical status, red cell fatty acid composition and membrane microviscosity in infants with atopic dermatitis. Drugs Exp Clin Res. 1994;20:77-84.

66. Humphreys F, Symons JA, Brown HK, et al. The effects of gamolenic acid on adult atopic eczema and premenstrual exacerbation of eczema. Eur J Dermatol. 1994:4:598-603.

67. Kiehl R, Ionescu G, Manuel P, et al. Clinical, immuno- and lipid modulating effects of unsaturated fatty acids in patients with atopic eczema. Z Hautkr. 1994;69:42-48.

68. Hederos CA, Berg A. Epogam evening primrose oil treatment in atopic dermatitis and asthma. Arch Dis Child. 1996;75:494-497.

69. Guenther L, Wexler D. Efamol in the treatment of atopic dermatitis. J Am Acad Dermatol. 1987;17:860.

70. Soyland E, Funk J, Rajka G, et al. Dietary supplementation with very long-chain n-3 fatty acids in patients with atopic dermatitis. A double-blind, multicentre study. Br J Dermatol. 1994;130:757. 64.

71. Gimenez Amau A, Barranco C, Alberola M, et al. Effects of linoleic acid supplements on atopic dermatitis. Adv Exp Med Biol. 1997:433285-9.

72. Wright S, Bolton $\mathrm{C}$. Breast milk fatty acids in mothers of children with atopic eczema. $\mathrm{Br} \mathrm{J}$ Nutr. 1989;62:693-7. 
General Discussion 
The causes for the increasing prevalence of allergic diseases over the last few decades in the Western world remain largely unknown. It is generally believed that this increase is caused by changes in the environment. Plausible causative factors should be able to influence the immune system (biological plausibility) and distributed in such a way that the reported differences can explain the differences in atopic disease within a population. Essential fatty acids may play a role in the development of atopic diseases and fulfill the above-mentioned criteria for a cause-and-effect relationship. This final chapter reflects on the findings of the EFAtop study, as presented in the previous chapters, in view of the delta-6desaturase (D6D) hypothesis and recently published studies. Also methodological aspects of the study design of the project will be discussed.

\section{EVOLUTION OF HYPOTHESES REGARDING EFAs IN ATOPIC DISEASE}

When the protocol for the present study was elaborated, the main hypothesis regarding essential fatty acids (EFAs) and atopy was the D6D hypothesis, which states that an impaired D6D enzyme activity is involved in the etiology of atopic disease. This impairment of D6D would result in higher LA levels and lower n-6 LCPs. As the n-6 LCPs are precursors of prostaglandin E1 (PGE1) and PGE2, this results in a disturbance in the balance between the various PGEs. Higher LA levels and lower n-6 LCP levels between atopic and non-atopic subjects have long been recognized in observational studies. Galli' was the first to show that these differences in n-6 EFAs precede the development of atopic diseases in newbom infants at risk for atopy. Other studies with infants and lactating mothers showed that breast milk from mothers whose infants subsequently developed atopic dermatitis contained less n6 LCPs than milk from mothers whose infants remained unaffected. ${ }^{2.3}$

While the present study was performed, new hypotheses emerged concerning EFAs and atopy. First, Black and Sharpe ${ }^{4}$ put forward the hypothesis that a shift in the dietary ratio of $n$ 6 and n-3 EFAs over the last decades had led to an increase of atopic diseases. These changes in EFA intake were a result of increased linoleic acid intake, mostly margarines and vegetable oils, and a decrease of n-3 EFA intake, mostly a reduction in the intake of oily fish. ${ }^{4}$ This $n-6 / n-3$ hypothesis was supported by several observational studies, which showed that a higher intake of margarine and a lower intake of butter were associated with allergic sensitization in infants. ${ }^{5.6}$ One study showed that these nutritional changes preceded the development of atopic disease. ${ }^{6}$ As a plausible biological explanation for the $n-6 / n-3$ hypothesis, Black and Sharpe ${ }^{4}$ stated that a higher intake of n-6 EFAs would result in higher levels of AA. PGE2 and leukotriene (LT) B4, eicosanoids derived from AA, are important factors promoting atopic inflammation. Furthermore, PGE2 increases the formation of IgE from T lymphocytes. ${ }^{7}$ On the other hand, the n-3 EFAs EPA and DHA competitively inhibit the formation of prostaglandins and leukotrienes derived from $A A^{7.8}$

Second, the intake of trans fatty acids has been found to be positively associated with the prevalence of atopic symptoms in infants. ${ }^{9}$ This was explained by a negative influence of trans fatty acids on the desaturation and chain elongation of n-6 and n-3 EFAs. ${ }^{10.11}$ As a result, a high intake of trans fatty acids would result in lower tissue levels of n-6 and n-3 LCPs. However, in observational studies it will be difficult to discern between the role of dietary $n-3 / n-6$ balance and trans fatty acids intake, because until recently a high intake of $n$ 6 EFAs by margarines also meant a higher intake of trans fatty acids from the same source. 


\section{FINDINGS OF THE EFA-TOP STUDY}

As mentioned before, our studies of the role of EFAs in atopic disease were based on the D6D hypothesis. This hypothesis was approached in three different ways. Firstly, we performed an observational study in 64 breast-fed infants to assess the relation between perinatal EFA status of the infant and atopic outcomes in the infant at one year of age (chapter 2). Secondly, we performed a randomized trial in 121 bottle-fed infants who were supplemented with gamma-linolenic acid (GLA,18:3n-6) or placebo from one week to 6 months of age in order to study the effect of GLA supplementation on atopic outcomes at one year of age (chapter 3). If D6D desaturase were impaired in infants prone to develop AD, circumventing this enzymatic step by GLA supplementation would result in fewer cases of AD. Thirdly, earlier trials of GLA supplementation, also based on the D6D hypothesis, were systematically reviewed in a meta-analysis (Chapter 5).

The observational study gave no evidence to support the D6D hypothesis. No consistent differences in n-6 levels in the first year of life could be shown between infants with and without $A D$ at age one year. As a measure of $D 6 D$ activity, we defined two D6D metabolic indices according to a procedure previously used by Businco. ${ }^{3}$ To this end, we calculated the ratio of LA to GLA and its LCPs, the metabolic n-6 index, as well as the ratio of ALA to $18: 4 n$ 4 and its LCPs, the so-called metabolic n-3 index. Results showed no differences between atopic and non-atopic individuals at the age of one year. Because D6D is also involved in the conversion of adrenic acid $(22: 4 n-6)$ to Osbond acid $(22: 5 n-6)$ and of DPA $(22: 5 n-3)$ into DHA (22:6n-3), the interpretation of the D6D index is not straightforward. Therefore, evaluation of the pattern of individual EFAs remains necessary. However, again, such evaluation of individual EFAs did not yield a pattern consistent with the D6D hypothesis.

It might be argued that the lack of a statistically significant relationship between EFA status and atopic outcomes could have been due to a type 2 error, in other words by a lack of statistical power of our study. However, Galli et al.,' who did find a relationship between lower levels in n-6 LCPs in the first three months of life and atopic outcomes at age one year, included approximately the same number of subjects as we did $\left(n=57\right.$; whereas Businco ${ }^{3}$ and Wright ${ }^{2}$ included 52 and 47 infants, respectively). These three studies showed a consistent relationship between lower levels of n-6 LCPs and the incidence of atopic disease and therefore we had a reasonable expectation to detect similar results in our study. The discrepancies between our observational findings and those of Galli, Wright and Businco could have been a result of differences in consumption of $n-6$ EFAs between populations: both Galli' and Businco ${ }^{3}$ had an Italian-based population, while Wright ${ }^{2}$ had a Zimbabweanbased population. In the Netherlands the LA intake is relatively high compared to other European countries. ${ }^{12}$ Therefore, the supply of $n-6$ EFA in our study population might have been so high for all subjects that insufficient contrast between subjects is left to detect an effect on atopy. Recent studies from North European countries ${ }^{7,13,14}$ also showed relations between EFAs and the development of atopy that were not strictly consistent with the D6D hypothesis. The choice of pregnant, atopic mothers as our study population might also have influenced the outcome of our study. By doing so we increased the number of infants prone to develop atopic disease, thus increasing the power of the study. However, by this way of selecting participants, it is possible that we simultaneously discarded part of the variability in 
EFA status amongst mother and infants, thereby reducing the power to detect an effect on atopy.

The randomized controlled trial (RCT) showed that increasing GLA levels in plasma phospholipids in the first three months of life were strongly negatively associated with the SCORAD at the age of one year. The intention-to-treat analysis showed a non-significant favorable trend for the clinical outcomes AD and SCORAD. No effect was shown on total serum IgE. The brands of infant formulas used by our study participants at the time of our study were the most commonly used ones and did not include LCP formulas such as marketed for preterm infants. Although the exact EFA composition between the formulas for term infants varied, with $n-6 / n-3$ ratios between individual formulas varying from $10: 1$ to $6: 1$, they contained only LA and ALA but no n-6 and n-3 LCPs at the time of performing our study. Because the formulas contained no GLA, the only source of GLA was our trial supplement. This provided maximum contrast between the group of infants who were supplemented with GLA and those supplemented with placebo. Because the intention-to-treat analysis did not provide statistically significant results, while on the other hand the explanatory analysis did, we have suggestive evidence but no definite proof of a causal effect of GLA supplementation on the severity of AD; The results could also be explained by a metabolic difference in EFA metabolism between infants with and without atopy, that only comes to expression after GLA supplementation. This comes close to the idea of a constitutional metabolic influence such as also proposed under the D6D hypothesis, but this would only be consistent with the D6D hypothesis in situations where LA supply is low, and not with the currently high LA intake of the population.

Our meta-analysis gave no clear support for the efficacy of GLA supplementation in subjects with $A D$.

Most studies were of low quality, included low numbers of subjects, and/or had an inadequate description of the results. Therefore, we had to use very conservative assumptions when calculating the pooled effect size of GLA supplementation on AD. As a result, we cannot exclude a type 1 error, i.e. that despite the lack of statistically significant results, an effect of GLA supplementation on severity of $A D$ is present.

In chapter 4 we hypothesized that DHA and other EFAs might play a role in the relationship between parity and neonatal IgE levels. Cord blood $\mathrm{IgE}$ is found to be a good indicator for later development of atopy. ${ }^{15}$ Our results show that matemal DHA levels in plasma phospholipids at 34-36 weeks of gestation are negatively related with parity. DHA levels in the mother, however, were not related to neonatal IgE levels. We found that decreased maternal LA levels and increased maternal AA levels were associated with increased neonatal IgE levels. This is contradictory to the D6D hypothesis. Neonatal n-3 EFAs were also positively or negatively related with neonatal IgE levels, but no consistent pattern was present that was consistent with either the D6D or the $n-6 / n-3$ hypothesis.

In conclusion, we have found only limited support for the D6D hypothesis. The results from the observational study and from chapter 4 did not support this hypothesis. The positive results from the RCT and the results from the meta-analysis do not exclude that supplementing GLA has a beneficial effect on (later) severity of AD, and therefore do not 
disprove the D6D hypothesis. In contrast with these findings, the results of the observational study suggest that severity of atopic dermatitis at age one year is related to reduced levels of individual $\mathrm{n}-3$ fatty acids, in particular a relative shortage of DHA over the first three months of life. This finding is consistent with the $n-6 / n-3$ hypothesis. As an operationalization of this hypothesis, we also constructed an index of perinatal $n-6 / n-3$ balance, but this index was not related to atopic outcomes.

At this moment the alleged impairment of the D6D enzyme system has been refuted by a study of a group in Sweden as well. ${ }^{16}$ In other publications from this group it was stated that an abnormal metabolism of n-3 and n-6 EFAs are related to atopic disease, ${ }^{7,13,14}$ but these conclusions were not very well supported by their evidence. The interpretation of these studies is hampered by the multi-comparison problem: because so many relations between individual fatty acids and atopic outcomes are studied in different biological samples (plasma of infants and mothers, breast milk) and different subgroups (atopic and non-atopic mothers), the likelihood of chance findings is very high. We tried to control this problem by a hypothesis driven approach (construction of specific indices as operationalization of the hypotheses), by looking for consistent patterns over time, between samples and between subgroups. In a review on EFAs in early atopic development, it was noted that individual fatty acids were related to atopic outcomes rather than $n-6$ or n-3 LCPs as a group. ${ }^{8}$ Our findings of associations between individual EFAs and severity of $A D$ are consistent with this new hypothesis, but the hypothesis lacks sufficient specificity to verify or falsify, and does not tell whether the variation in EFA status is due to metabolic or dietary variation (or its interaction). From a pathogenetic point of view it has been suggested that the implicated EFAs are the n6 and $n-3$ precursors of the less inflammatory immune mediators (GLA as a precursor for PGE1; and EPA as a precursor for PGE3 and 5-series LTs), rather than n-3 and n-6 LCPs as a group or their parent fatty acids LA and ALA since they include more remote precursors. ${ }^{8}$ Our results are supportive for this idea.

Further evidence for a role of relative deficiency of specific dietary n-3 EFA intake in the aetiology of atopy may come from trials of $n-3$ supplementation in western countries where n3 EFA intake is low. These trials have been motivated by the anti-inflammatory action of $n-3$ fatty acids. Two systematic reviews of $n-3$ EFA supplementation in asthma ${ }^{17,18}$ showed no clear evidence of effectiveness. None of the GLA or n-3 trials have focussed on early development of atopy in infants. The same was true for the GLA trials. As far as we know, our GLA trial was the first preventive trial. Therefore, the anti-inflammatory role of specific EFAs such as GLA and EPA may only be relevant to prevent severe atopic dermatitis when the infants EFA status is adequate at an early enough age, before atopic manifestations develop and skin inflammation starts to be chronic.

The results of both our observational study and RCT suggest that EFAs play no direct role in the $\mathrm{IgE}$ mediated component of atopy or in the prevention of $\mathrm{AD}$, but do exert an influence on the severity of the expression of atopy in the first year of life. Our findings are in concordance with the results presented by Ziboh et al. ${ }^{19-21}$ who suggested that certain EFAs might play a role in the severity of $A D$, rather than exerting an effect on $\operatorname{lgE}$. In these studies Ziboh et al. show that dietary GLA is actively converted into DGLA in the skin. Because delta-5desaturase (which converts DGLA into AA) is absent in skin epidermis, the increase of DGLA in the skin as result of GLA supplementation will not result in an increase in AA and PGE2 in the skin. As a result, it was predicted that supplementing human with GLA raises PGE1 and 
15-hydroxy-eicosatrienoic acid (15-HETrE) levels. Because PGE1 and 15-HETrE both have anti-inflammatory properties, supplementation with GLA might alleviate the severity of AD. Many of the immunological changes associated with GLA supplementation have also been attributed to alterations in GLA metabolites that in turn downregulate the production of AAderived leukotrienes. ${ }^{8}$ For instance, Ziboh and Fletcher showed that GLA inhibits LTB4 in a dose-dependent way, ${ }^{20}$ but these results have not been confirmed in later studies. ${ }^{22}$

\section{METHODOLOGICAL CONSIDERATIONS REGARDING THE MEASUREMENT OF ATOPIC OUTCOMES}

To assess whether the infants had developed $A D$ in the first year of life, we used the UK Working party probability score. We believe that even though the UK Working party probability score is a good measure to discern between infants who develop AD and those who do not, in our study it may have caused a considerable loss of specificity because two of the seven criteria that can be fulfilled were fulfilled by all participants; i.e. (1) a positive family history was our main inclusion criterion and (2) onset before the age of 2 years.

The SCORAD was used to assess severity of AD because it was the only validated list of the severity of AD, which was available at the start of our study. In the observational study and in the RCT we found consistent results between EFAs and the SCORAD, but not between EFAs and the UK Working party probability score. We also used these outcome variables in a study where we looked at parasite infections and AD (not presented in this thesis). Also in this study, SCORAD was the only outcome measure that showed significant results between infants infected with certain parasites and those who were not. A further difference between the UK working party probability score and the SCORAD as outcome measures for atopic dermatitis lies in their statistical properties. The UK working party probability score showed a bimodal distribution suggesting a clear-cut dichotomising between high probability score (atopy present) and a low score (atopy absent). The effect is then measured in the statistical analysis as a comparison between two categories (and a logistic regression model). This has generally lower statistical power compared to outcomes measured on a continuous scale, especially if a dose-effect relationship is present. The SCORAD is measured on such a continuous scale, and therefore the SCORAD may be more responsive for the effect of GLA supplementation and especially the dose-effect relationship with the increase of plasma GLA level.

We found that the clinical outcomes (AD (as calculated from the UK Working party probability score) and SCORAD) were not correlated with total serum IgE. This indicates that different components are involved in the pathogenesis of $A D$. Recently a new nomenclature for $A D$ has been suggested: the atopic eczema/dermatitis syndrome (AEDS). ${ }^{23}$ According to these authors this syndrome exists of an allergic AEDS and a nonallergenic AEDS. The allergic AEDS is further divided in an IgE-associated AEDS and a non-lgE-associated AEDS. We did not divide the infants with $A D$ into either one of these sub-classifications because we think that studying the pathogenetic components of $A D$ is more rewarding than using a classification made for clinical but not etiological purposes.

Other, for instance recent studies in Sweden and Finland ${ }^{13.14 .24 .26}$ used composite indices of atopic outcomes: children with at least one positive skin prick test and symptoms of AD, urticaria, or bronchial asthma. In this way they narrowed their atopic population to infants 
with allergic AEDS, while our group also included infants with nonallergenic AEDS according to the definition by Johansson $e t$ al. ${ }^{23}$ The heterogeneity of patients in terms of AEDS between the Finnish and Swedish studies on one hand and ours on the other hand might partially explain the inconsistent findings between our studies and theirs.

\section{CONSIDERATIONS FOR PREVENTION AND FUTURE RESEARCH}

Longer breast-feeding is recommended in infants at risk of developing atopic diseases and has proven to be effective in the prevention of atopic diseases. However, recently a study has shown that this may not be true for a subgroup of mothers with high IgE levels; their infants had a higher risk of $A D$ when breast-fed compared to formula fed. ${ }^{27.28}$ The authors suggested that this may be due to differences in immune factors such as cytokines but also to relative deficiency of EFA levels in breast milk. Our results confirm the importance of perinatal EFAs. In a previous study from our group we found a decreased level of GLA, DGLA and most notably of AA in breast milk in atopic compared to non-atopic mothers, suggesting involvement of D6D. Supplementation of atopic mothers with GLA increased GLA levels of plasma phospholipids but it did not restore the level of AA. ${ }^{29}$

Since the D6D hypothesis is not confirmed, and regarding other hypotheses concerning EFAs in relation to atopy, it may be worthwhile to explore the association between EFAs in breast milk and maternal atopic constitution. Also it has been suggested recently that EFAs play a role in local gut immunity and adhesion of gut microbial flora. ${ }^{30}$ The role of gut microflora in the protection against atopy, provided by breastfeeding, has drawn recent attention $^{31}$ and EFAs in breast milk may be interesting to study among the many factors that influence the infants gut flora and immunity.

Currently guidelines on dietary intake of EFAs are being discussed for pregnant and breastfeeding women to establish a desired EFAs status in the infant, e.g. weekly eating fatty fish or use of fish oil supplements. The most important reason is the role of EFAs in the neurological and retinal development. The immune effects on the infant are only recently being explored, including atopic disease. The beneficial effects should be balanced against possible adverse effects due to changes of prostaglandin levels, resulting for instance in a longer duration of pregnancy. ${ }^{32}$ Also, fatty fish may contain relatively high levels of dioxins, ${ }^{33.34}$ and the health effects on the child are largely unknown.

Interestingly, Karmaus has put forward that the protecting effect of parity on the risk of atopy may be due to decreasing level of dioxins and related organochlorines in maternal fat by repeated fat mobilization with repeated pregnancies and lactation. ${ }^{35}$ This is an interesting hypothesis that has the potential to explain the increase of atopic disease in the Westernised countries, and urgently needs evaluation because of the increasing exposure to these agents in developing countries.

Some mothers cannot or will not breast-feed their infants and therefore their offspring will be dependent on infant formula for EFA supply. Many brands of infant formula still contain only LA and ALA and only some brands have also been added AA and DHA. Many studies have been and are being performed to assess whether LCPs, especially n-3 LCPs, should be added to current formulas. Usually the EFA composition of breast milk from well-nourished mothers is taken as golden standard. It is still not clear, however, what the recommended 
dietary intake levels for the separate EFAs are, and with which specific (functional) outcomes these recommendations will be linked. ${ }^{36.37}$ Furthermore, supplementation of infant formula with specific n-6 and/or n-3 LCPs solely is not uncontroversial because the eicosanoids from the $n-3$ EFAs may induce mitogenic responses and have an influence on the maturation and activity of the immune system ${ }^{30}$ and because a high intake of $n-6$ and/or n-3 LCPs may increase in vivo lipid peroxidation. ${ }^{30}$

Our conclusion for future research is as follows:

More research is needed to investigate whether GLA and/or EPA and DHA have positive effects on the development of $A D$, to study possible side effects, and to establish the optimal amounts of DHA and EPA to be added to formula. To find out whether EPA or DHA have a beneficial effect on $A D$ in infancy, the trials with neurological outcomes in $n-3$ LCP supplementation studies could include atopy as secondary outcome. Longer follow-up of observational studies and trials in infants should be performed in order to assess whether the observed differences in blood EFA levels and atopic diseases in the first year of life continue over a longer period of time. A possible alternative would be performing observational studies in which the EFA consumption and EFA levels in blood are measured accurately over a longer period of time; however, from today's questionnaires EFA intake cannot be estimated accurately. The development of tools for measurements of EFA consumption and outcome measures of atopic disease is therefore an important issue for further research.

\section{REFERENCES}

1. Galli E, Picardo M, Chini L, et al. Analysis of polyunsaturated fatty acids in newborn sera: a screening tool for atopic disease? Br J Dermatol. 1994;130:752-6.

2. Wright $\mathrm{S}$, Bolton $\mathrm{C}$. Breast milk fatty acids in mothers of children with atopic eczema. $\mathrm{Br} \mathrm{J}$ Nutr 1989;62:693-7.

3. Businco L, loppi M, Morse NL, et al. Breast milk from mothers of children with newly developed atopic eczema has low levels of long chain polyunsaturated fatty acids. J Allergy Clin Immunol. 1993;91:1134-9.

4. Black PN, Sharpe S. Dietary fat and asthma: is there a connection? Eur Respir J. 1997:10:6-12.

5. Bolte G, Frye C, Hoelscher B, et al. Margarine consumption and allergy in children. Am J Respir Crit Care Med. 2001;163:277-9.

6. Dunder T, Kuikka L, Turtinen J, et al.. Diet, serum fatty acids, and atopic diseases in childhood. Allergy. 2001;56:425-8.

7. Duchen K, Bjorksten B. Polyunsaturated n-3 fatty acids and the development of atopic disease. Lipids. 2001;36:1033-42.

8. Kankaanpaa P, Sutas Y, Salminen S, et al. Dietary fatty acids and allergy. Ann Med. 1999;31:2827.

9. Weiland SK, von Mutius E, Husing A, et al. Intake of trans fatty acids and prevalence of childhood asthma and allergies in Europe. ISAAC Steering Committee. Lancet. 1999;353:2040-1.

10. Cook HW. The influence of trans-acids on desaturation and elongation of fatty acids in developing brain. Lipids. 1981:16:920-6.

11. Cook HW, Emken EA. Geometric and positional fatty acid isomers interact differently with desaturation and elongation of linoleic and linolenic acids in cultured glioma cells. Biochem Cell Biol. 1990;68:653-60.

12. Hulshof KF, van Erp Baart MA, Anttolainen M, et al. Intake of fatty acids in western Europe with emphasis on trans fatty acids: the TRANSFAIR Study. Eur J Clin Nutr. 1999;53:143-57.

13. Yu G, Kjellman NI, Bjorksten B. Phospholipid fatty acids in cord blood: family history and development of allergy. Acta Paediatr. 1996;85:679-83.

14. Yu G. Bjorksten B. Serum levels of phospholipid fatty acids in mothers and their babies in relation to allergic disease. Eur J Pediatr. 1998;157:298-303. 
15. Edenharter G, Bergmann RL, Bergmann KE, et al. Cord blood-lgE as risk factor and predictor for atopic diseases. Clin Exp Allergy. 1998;28:671-8.

16. Duchen K. Are human milk polyunsaturated fatty acids (PUFA) related to atopy in the mother and her child? Allergy. 2001:56:587-92.

17. Smit HA, Grievink L, Tabak C. Dietary influences on chronic obstructive lung disease and asthma: a review of the epidemiological evidence. Proc Nutr Soc. 1999;58:309-19.

18. Woods RK, Thien FC, Abramson MJ. Dietary marine fatty acids (fish oil) for asthma. Cochrane Database Syst Rev. 2000:CD001283.

19. Ziboh VA. Prostaglandins, leukotrienes, and hydroxy fatty acids in epidermis. Semin Dermatol. 1992:11:114-20.

20. Ziboh VA, Fletcher MP. Dose-response effects of dietary gamma-linolenic acid-enriched oils on human polymorphonuclear-neutrophil biosynthesis of leukotriene B4. Am J Clin Nutr.1992;55:3945.

21. Ziboh VA, Miller CC, Cho Y. Metabolism of polyunsaturated fatty acids by skin epidermal enzymes: generation of antiinflammatory and antiproliferative metabolites. Am J Clin Nutr. 2000;71:361s-6s.

22. Lehmann B, Huebner C, Jacobi $\mathrm{H}$, et al. Effects of dietary gamma-linolenic acid-enriched evening primrose seed oil on the 5-lipoxegenase pathway of neutrophil leukocytes in patients with atopic dermatitis. J Dermatolog Treat. 1995;6:211-218.

23. Johansson SG, Hourihane JO, Bousquet J, et al. A revised nomenclature for allergy: An EAACI position statement from the EAACI nomenclature task force. Allergy. 2001;56:813-824.

24. Duchen K, Casas R, Fageras-Bottcher M, et al. Human milk polyunsaturated long-chain fatty acids and secretory immunoglobulin $\mathrm{A}$ antibodies and early childhood allergy. Pediatr Allergy Immunol. 2000:11:29-39.

25. Duchen K, Yu G, Bjorksten B. Atopic sensitization during the first year of life in relation to long chain polyunsaturated fatty acid levels in human milk. Pediatr Res. 1998;44:478-84.

26. Kankaanpaa P. Nurmela K, Erkkila A, et al. Polyunsaturated fatty acids in maternal diet, breast milk, and serum lipid fatty acids of infants in relation to atopy. Allergy. 2001;56:633-8.

27. Wright AL, Stern DA, Halonen M. The association of allergic sensitization in mother and child in breast-fed and formula-fed infants. Adv Exp Med Biol. 2001;501:249-55.

28. Wright AL, Holberg CJ, Taussig LM, et al. Factors influencing the relation of infant feeding to asthma and recurrent wheeze in childhood. Thorax. 2001;56:192-7.

29. Thijs C, Houwelingen A, Poorterman I, et al. Essential fatty acids in breast milk of atopic mothers: comparison with non-atopic mothers, and effect of borage oil supplementation. Eur J Clin Nutr. 2000:54:234-8.

30. Donnet-Hughes A, Schiffrin EJ, Turini ME. The intestinal mucosa as a target for dietary polyunsaturated fatty acids. Lipids. 2001;36:1043-52.

31. Kankaanpaa PE, Salminen SJ, Isolauri E, et al. The influence of polyunsaturated fatty acids on probiotic growth and adhesion. FEMS Microbiol Lett. 2001;194:149-53.

32. Allen KG, Harris MA. The role of n-3 fatty acids in gestation and parturition. Exp Biol Med (Maywood). 2001;226:498-506.

33. Jensen E, Bolger PM. Exposure assessment of dioxins/furans consumed in dairy foods and fish. Food Addit Contam. 2001;18:395-403.

34. Svensson BG, Nilsson A, Hansson M, et al. Exposure to dioxins and dibenzofurans through the consumption of fish. N Engl J Med. 1991;324:8-12.

35. Karmaus W, Botezan C. Does a higher number of siblings protect against the development of allergy and asthma? A review. J Epidemiol Community Health. 2002;56:209-17.

36. Gibson RA, Makrides M. Long-chain polyunsaturated fatty acids in breast milk: are they essential? Adv Exp Med Biol. 2001:501:375-83.

37. Gibson RA, Makrides M. n-3 polyunsaturated fatty acid requirements of term infants. Am J Clin Nutr. 2000;71:251S-5S.

38. Sellmayer A, Koletzko B. Long-chain polyunsaturated fatty acids and eicosanoids in infants-physiological and pathophysiological aspects and open questions. Lipids 1999;34:199-205.

39. Eritsland J. Safety considerations of polyunsaturated fatty acids. Am J Clin Nutr. 2000;71:197S201S. 


\section{SUMMARY}

Atopic diseases (atopic dermatitis/ milk scab, food allergy, hay fever and other airway allergies, and allergic asthma) in the Western world has increased two- to threefold over the last three decades. The role of essential fatty acids in the development of atopic dermatitis is subject of this thesis. Essential fatty acids (EFAs) are poly-unsaturated fatty acids from the $n$ 3 and $n-6$ families which, regarding their role in the human body, are indispensable. The two main EFAs linoleic acid and alphalinolenic acid, cannot be synthesized de novo en have to be taken up from the diet. Humans are able to process these EFAs into even more elongated and more unsaturated fatty acids. When we refer to EFAs in this thesis we do not only mean LA and ALA but also their more elongated and more unsaturated fatty acids of the same family.

In chapter 1 it is described how these EFAs can be involved in the etiology of atopic diseases. In the n-6 EFA family, linoleic acid, derived from food, is subsequently converted into gamma-linolenic acid (GLA) and longer-chain polyenes (LCPs) such as dihomo-gammalinolenic acid and arachidonic acid. Although LCPs of the n-3 EFA family can be synthesized from alpha-linolenic acid (ALA), the major source of n-3 LCPs is food. In the 1930s, lower levels of arachidonic acid, an n-6 LCP, were reported in the serum of children with atopic dermatitis compared to healthy children. More recent studies have shown higher levels of LA and substantially lower levels of its LCPs in the blood of patients with atopic dermatitis. As an explanation for these findings a reduced conversion of linolenic acid into gamma-linolenic acid and subsequent LCPs was suggested, possibly as a consequence of an impaired activity of the enzyme delta-6-desaturase (D6D-hypothesis).

In the early nineteen nineties, Galli and co-workers found that lower n-6 LCP levels in umbilical cord blood preceded the development of atopic dermatitis in newborn infants with a family history of atopic disease. Other studies showed that breast milk from mothers whose infants subsequently developed atopic dermatitis contained less n-6 LCPs than milk from mothers whose infants remained unaffected. Furthermore, it became clear that prolonged breast feeding protects the infant against the development of atopic diseases. Unlike breast milk, infant formulas for term neonates until recently contained only linoleic acid and alphalinolenic acid as EFAs but no LCPs.

In this thesis the results of a study is described (which we have named the EFAtop study. after 'EFAs' and 'atopy') to study the role of EFAs in the etiology of atopic diseases. assuming a diminished activity of the D6D (D6D-hypothesis). Meanwhile a new hypothesis was formulated by Black and Sharpe suggesting that the $n-6 / n-3$ ratio in the current diet might have been the base of the increasing rise of atopic diseases in the past decades. In West European countries the consumption of n-6 and n-3 EFAs has changed considerably as a result of increased linoleic acid intake, mostly from margarines and vegetable oils, and a decrease of $n-3$ EFA intake, mostly a reduction in the intake of fatty fish. This role of the ratio of dietary n-6/n-3 EFAs was supported by several observational studies, which showed that a high intake of margarine and a low intake of butter were associated with atopic diseases in infants. We were able to test this hypothesis in the EFAtop study

The EFAtop study comprised two major studies, an observational study as described in chapter 2 and a randomized controlled clinical trial, as described in chapter $\mathbf{3}$. In both studies, atopic, pregnant women were included at 34-36 weeks of gestation. Main outcome 
measures were presence of atopic dermatitis (as assessed by the UK Working Party Criteria) and severity of atopic dermatitis (as assessed by the SCORAD). Apart from these clinical outcome measures we measured patho-biological markers of the atopic constitution: total and specific IgE. Allergic symptoms are usually based on the formation of IgE antibodies against normal substances that are present in the air or in food (specific IgE; IgE-mediated hypersensitivity); while higher total IgE levels in the blood serum in early life a marker is for an atopic constitution. Total serum IgE was measured in the neonate at one week of age as a predictor of later atopic outcomes. EFA status and $\operatorname{lgE}$ status of the mothers were assessed at entry. In the neonates EFA status of plasma phospholipids was assessed at one week, three months, six months and one year of age.

The observational study described in chapter 2 aimed at confirming the study by Galli et al. who showed that early changes in EFA status preceded the development of atopic dermatitis. In our study 64 mothers and their infants were followed from 34-36 weeks of gestation until the infant reached the age of one year. All mothers breast-fed their infants for at least 4 weeks. As a measure of D6D activity, we calculated two D6D metabolic indices from the EFA levels, for the n-6 EFAs and the n-3 EFAs respectively. At the same time we looked at the ratio between the $n-6 / n-3$ EFAs and at two indices that are a measure for $n-3$ status, the DHA sufficiency index and the DHA deficiency index. Twenty-five children developed atopic dermatitis at the age of one year. Both D6D indices showed no relation with the atopic outcomes. Our study did not confirm the lower levels of n-6 LCPs in breast milk from mothers of atopic children as previously reported. However, in the infants with the severest dermatitis compared to the least affected group the docosahexaenoic acid (DHA) sufficiency index was $53 \%$ lower in mothers prepartum, $31 \%$ lower in the infant at one week and $58 \%$ lower at three months. These results do not support the D6D-hypothesis, but suggest that lower levels of n-3 LCPs worsen the development of atopic dermatitis. This is partially in line with the $n-6 / n-3$ hypothesis. Because our results are not in concordance with previous studies (performed in Italy and Zimbabwe; including the study by Galli) we speculate that these differences are a result of a higher intake of linoleic acid and a higher ratio of $n-6 / n-3$ EFAs in the intake of EFAs in the Netherlands.

Results from our study are not consistent with the hypothesis that an inborn defect of the D6D enzyme system in mothers or infants causes atopic disease. Our results do support a potential role in the etiology of atopic dermatitis of a relative deficiency of n-3 LCP intake by the mother, which determines n-3 status in the infants through intra-uterine supply and lactation.

Chapter 3 presents the design and the results of a randomized controlled trial (RCT). The expectation was tested that dietary supplementation of formula with borage oil, which is rich in GLA, in the first six months of life, in a dosage comparable to the intake by breastfeeding, might prevent the development of atopy. The expectation is predicted by the D6Dhypothesis, because GLA is the first product of LA in the chain of $n-6$ EFAs, and supplementation is supposed to circumvent the impaired D6D desaturation. Participants were 118 formula fed infants from mothers with a history of atopic diseases. The infants were randomly assigned to either GLA supplementation or to placebo supplementation. The intention-to-treat analysis showed that the risk of atopic dermatitis and severity of dermatitis at one year tended to be lower in the GLA supplemented group compared to the placebo 
group. Also secondary measures of atopic dermatitis (itch, and use of topical steroids) tended to be lower in the GLA supplemented group. However, the effect was smaller than predicted and did not reach statistical significance.

The level of GLA in blood plasma doubled in supplemented infants compared to the placebo group. We used this increase of GLA in plasma phospholipids as a marker of compliance and investigated whether this increase correlated with the atopic outcomes. This explanatory analysis showed that a high increase in GLA level between one week and 3 months of age was associated with a statistically significant lower dermatitis severity score at the age of one year. Because this result is not based on an-intention-to-treat analysis causal proof is not ensured and biases may be present. The results were systematically checked for biases but none could be demonstrated. Also, the result could be explained by a metabolic difference in EFA metabolism between infants with and without atopy, which only comes to expression after GLA supplementation. However, we do not find this plausible because this does not fit in one of the hypotheses. We conclude that supplementing infants at risk of developing atopic dermatitis with GLA may alleviate the severity of atopic dermatitis, but we cannot confirm this with causal evidence.

We found remarkable similarities in the observational study and the RCT with respect to the outcome measures. The results of both our observational study and RCT (chapters $\mathbf{2}$ and 3 ) suggest that EFAs are related to the severity of atopic dermatitis, but not to total or specific IgE. Therefore, EFAs play no direct role in the IgE mediated component of atopy, but do exert an influence on the of the severity of dermatitis in the first year of life, probably as result of anti-inflammatory effects of EFAs such as GLA and n-3 EFAs. This anti-inflammatory effect is also suggested by previous studies, which have shown that GLA supplementation increases the levels of anti-inflammatory metabolites in the skin.

Other hypotheses have emerged in the last decade to explain the rising trend of atopic diseases, subsumed under the broader context of the 'hygiene-hypothesis'. Studies have shown that hay fever and atopic dermatitis occur less frequently in infants who have older brothers and/or sisters. This effect has been explained by the greater chances of crossinfection from older siblings in large families, which may have an important protective role in the expression of atopy: the so-called sibling effect. A recent study by Karmaus $e t$ al. showed that the effect of increasing birth order could already be shown in cord blood IgE levels. As the concentration of total IgE in umbilical cord blood was found in several studies to be predictive of later development of atopy, the sibling effect may have its origin (at least partly) in utero. If this 'intra-uterine sibling effect'-hypothesis is correct, also neonatal IgE would be expected to decrease with parity. We aimed to test this idea using neonatal serum IgE, instead of cord blood IgE as Karmaus did (chapter 4). We were able to confirm that increasing birth order is accompanied by lower total serum IgE levels at the age of one week. As EFA levels in the mother during pregnancy might influence the atopic outcomes in her infants we wanted to investigate whether intra-uterine EFAs play a role on total serum IgE at birth since also EFA levels in the mother during pregnancy are influenced by the number of pregnancies.

We did find that parity was associated with a lower DHA status of the mother, but not of the infant. According to the current hypothesis of n-6/n-3 EFAs a high level of n-3 EFAs would protect against high levels of $\mathrm{IgE}$ in the infant. We could not demonstrate such an effect on 
neonatal IgE. We did find seasonal influences that suggest an intra-uterine effect. Our findings suggested that pollen exposure in atopic, pregnant women confers intrauterine stimulation of fetal $\mathrm{IgE}$ production.

Studies of oral EFA supplementation to alleviate atopic dermatitis have been performed for some decades already using supplements with high GLA content from primrose oil or borage oil, or n-3 LCPs from fish oil. We have performed a meta-analysis of these studies (chapter 5) to estimate the effect of EFA supplementation on the severity of atopic dermatitis. The pooled effect size for GLA gave no clear support for the efficacy of GLA supplementation in subjects with atopic dermatitis. Most studies of GLA supplementation were of low quality, included low numbers of subjects, and/or had an inadequate description of the results. Therefore, we had to use very conservative assumptions when calculating the pooled effect size of GLA supplementation on atopic dermatitis. As a result, we cannot exclude a small beneficial effect of GLA supplementation on severity of atopic dermatitis. Fish oil supplementation showed no beneficial effect.

Chapter 6 discusses the methodology and results of the EFAtop study and the metaanalysis. We found that the clinical outcomes (diagnosis of atopic dermatitis and severity of atopic dermatitis) were not correlated with total serum $\operatorname{lgE}$. This indicates that other components are involved in the pathogenesis of atopic dermatitis at an early age. The results of both our observational study and the RCT suggest that EFAs play no direct role in the IgE mediated component of atopy or in the prevention of atopic dermatitis, but possibly do exert an influence on the inflammatory component of the atopic dermatitis as expressed by the severity of the dermatitis. Our meta-analysis gave no clear support for the efficacy of GLA supplementation in subjects with atopic dermatitis, but the studies included in the metaanalysis were mainly performed in adults with ongoing dermatitis. Therefore, an effect in infants is not excluded and this fits in the findings of previous studies that EFAs are mainly important in the development of atopy in the first months of life.

The risk of atopic dermatitis can be lowered by prolonged breastfeeding. However, for some women this is not an option. Therefore, adding n-6 and/or n-3 EFAs in artificial formula might be an option. Our study does not show convincingly that supplementation with GLA to formula is effective. In light of the $n-6 / n-3$ hypothesis too little is yet known about the optimal ratios between the EFAs for enrichment of formula. Finally the implications for future research are considered. At this moment studies are performed in which the effects of enrichment of formula with n-3 LCPs on neurological development in infants are studied.

To further explore a potential effect of different EFA formulation in formula on prevention of atopic dermatitis, these ongoing trials could include atopy as secondary outcome. Longer follow-up of observational studies and randomized controlled trials in infants should be performed in order to assess whether the results found in the first year are still present at later age. 


\section{SAMENVATTING}

In de laatste drie decennia is het voorkomen van atopische aandoeningen (zoals atopisch eczeem dauwworm, voedsel-allergieên, hooikoorts en andere luchtweg allergieèn, en allergisch astma) verdubbeld tot verdrievoudigd in de Westerse wereld. Dit proefschrift gaat over de rol van essentiēle vetzuren bij het ontstaan van atopische aandoeningen. Essentiêle vetzuren zijn meervoudig onverzadigde vetzuren van de $n-3$ en n-6 families, die gezien hun rol in het menselijk lichaam, onmisbaar zijn. De twee hoofdvetzuren, linolzuur en alfalinoleenzuur, kunnen niet door de mens zelf gemaakt worden en dienen uit het voedsel te worden opgenomen. Mensen zijn wel in staat om deze essentiële vetzuren verder om te zetten tot nog langere en meer onverzadigde vetzuren. Als in dit proefschrift verwezen wordt naar essentiêle vetzuren omhelst dit niet alleen linolzuur en alfa-linoleenzuur maar ook de meer verlengde en meer onverzadigde vetzuren van dezelfde familie. In hoofdstuk 1 wordt beschreven hoe deze essentiële vetzuren betrokken kunnen zijn in het ontstaan van atopische aandoeningen. In de n-6 familie, wordt linolzuur, opgenomen uit de voeding, omgezet in gamma-linoleenzuur (gamma-linolenic acid, GLA) en lange-keten meervoudig onverzadigde vetzuren (long chain polyenes, LCP's) zoals dihomo-gamma-linoleenzuur en arachidonzuur. Alhoewel de LCP's van de n-3 familie gemaakt kunnen worden uit alfalinoleenzuur, is de belangrijkste bron van n-3 LCP's het voedsel. In de dertiger jaren van de twintigste eeuw werd ontdekt dat serum van kinderen met atopisch eczeem (dauwworm) lagere spiegels van arachidonzuur, een n-6 LCP, bevatten dan gezonde kinderen. Meer recente onderzoeken in bloed van patiênten met atopisch eczeem hebben iets hogere spiegels aan linolzuur en wezenlijk lagere spiegels aan n-6 LCP's aangetoond. Als een verklaring hiervoor voerde men aan dat de omzetting van linolzuur naar GLA en de daarop volgende n-6 LCP's verminderd was, mogelijk als gevolg van een verminderde activiteit van het enzym delta-6-desaturase (D6D).

In het begin van de negentiger jaren van de twintigste eeuw toonden Galli en zijn medewerkers aan dat het navelstrengbloed van kinderen met een familiegeschiedenis van atopie die later atopisch eczeem kregen, lagere spiegels bevatte van n-6 LCP's dan van de kinderen die gezond bleven. Nog andere onderzoeken toonden aan dat de moedermelk van vrouwen wier kinderen vervolgens atopisch eczeem kregen, lagere spiegels aan n-6 LCP's hadden dan de moedermelk van moeders wier kinderen gezond bleven. Daarnaast werd het duidelijk dat langdurige borstvoeding bescherming bood tegen het ontstaan van atopisch eczeem bij kinderen.

In dit proefschrift wordt een onderzoek beschreven (die we het EFAtop-onderzoek zijn gaan noemen, naar 'EFA', essentiële vetzuren in het engels en 'atop' van atopie) naar de rol van essentiêle vetzuren bij het ontstaan van atopische aandoeningen, uitgaande van een verminderde activiteit van het D6D (de D6D-hypothese). Ondertussen was een nieuwe hypothese opgesteld door Black en Sharpe die suggereerden dat de verhouding van n-6 tot $\mathrm{n}-3$ vetzuren in het huidige voedselopname patroon de basis zou kunnen zijn voor de sterke stijging van atopische aandoeningen in de laatste decennia. In de Westerse landen is de consumptie van $n-6$ en $n-3$ vetzuren drastisch veranderd als gevolg van een verhoogde linolzuur inname, grotendeels uit margarines en plantaardige oliên, en een verlaagde inname van $n-3$ vetzuren, voornamelijk door een verminderde consumptie van vis. De rol van de ratio van $n-6 / n-3$ vetzuren werd bevestigd door andere onderzoeken waarin werd aangetoond dat 
een hoge consumptie van margarine en een lage inname van boter geassocieerd waren met atopische aandoeningen bij kinderen. Aan de hand van de gegevens uit het EFAtoponderzoek konden wij ook de $n-6 / n-3$ hypothese toetsen.

Het EFAtop onderzoek bestond uit twee onderzoeken, een observationeel onderzoek zoals beschreven in hoofdstuk 2, en een gerandomiseerd onderzoek, zoals beschreven in hoofdstuk 3. In beide onderzoeken werden atopische, zwangere vrouwen ingesloten in het onderzoek die 34-36 weken zwanger waren. De belangrijkste uitkomstmaten waren; de aanwezigheid van atopisch eczeem (vastgesteld met de UK Working Party Criteria) en de ernst van het eczeem (vastgesteld middels de SCORAD) op de leeftijd van één jaar bij het kind. Naast deze klinische uitkomstmaten namen we ook biologische markers voor atopie mee: totaal en specifiek IgE. Allergische verschijnselen zijn meestal gebaseerd op de vorming van IgE-antilichamen tegen veel voorkomende stoffen in de lucht of in de voeding (specifiek IgE; IgE gemedieerde overgevoeligheid); terwijl al vroeg in het leven een verhoogd totaal niveau van $\mathrm{IgE}$ in het bloedserum duidt op een atopische aanleg hiervoor. Totaal serum IgE werd bij het kind bepaald op de leeftijd van één week als voorspeller voor latere atopische aandoeningen.De vetzuurstatus en IgE status van de moeder werd vastgesteld bij opname in het onderzoek. Bij de zuigelingen werd de essentiële vetzuurstatus van het plasma vastgesteld op de leeftijden van één week, drie maanden, zes maanden en één jaar.

Het observationeel onderzoek zoals beschreven in hoofdstuk 2 had als doel het onderzoek van Galli et al., welke aantoonde dat vroege veranderingen in essentiële vetzuurstatus voorafgaan aan atopisch eczeem, te bevestigen. In ons onderzoek werden 64 moeders en hun kinderen gevolgd vanaf 34-36 weken zwangerschap tot het kind de leeftijd bereikte van één jaar. Alle kinderen kregen ten minste 4 weken uitsluitend borstvoeding. Als een maat voor D6D activiteit, berekenden we 2 indexen uit de essentiële vetzuurspiegels, voor de n-6 en de $n-3$ vetzuren afzonderlijk. Tegelijkertijd keken we naar de ratio van de $n-6 / n-3$ vetzuren en naar een tweetal indexen die de n-3 status beschrijven, de docosahexaenoic acid (DHA) sufficiency index en de DHA deficiency index. Bij 25 kinderen werd op de leeftijd van één jaar de diagnose atopisch eczeem vastgesteld. We vonden dat er geen samenhang bestond tussen deze indexen en de latere atopische uitkomsten. Ons onderzoek kon ook geen verband aantonen met de lagere spiegels van n-6 LCP's in de moedermelk van de moeders wier kinderen atopisch eczeem ontwikkelden, hetgeen in eerder onderzoek wel was gevonden. Echter, in de groep kinderen die het minst ernstige eczeem hadden in vergelijking tot de kinderen met het meest ernstige eczeem was de DHA sufficiency index $53 \%$ lager in de moeders tijdens de zwangerschap, $31 \%$ lager bij de kinderen op de leeftijd van één week en $58 \%$ lager in de kinderen op de leeftijd van drie maanden. Deze resultaten ondersteunen de D6D hypothese niet, maar suggereren dat lage spiegels van $n-3$ vetzuren de ontwikkeling van atopisch eczeem verergeren. Dit past gedeeltelijk bij de $n-6 / n-3$ hypothese. Over de discrepantie met eerder genoemde onderzoeken die waren uitgevoerd in Italiē en Zimbabwe, waaronder die van Galli, speculeren we dat dit te wijten is aan een hogere inname van linolzuur en een hogere ratio van de inname in $n-6 / n-3$ vetzuren in Nederland.

Resultaten van ons onderzoek komen niet overeen met de hypothese dat een aangeboren afwijking van het D6D enzym systeem in moeders of kinderen atopisch eczeem veroorzaakt. Onze resultaten geven wel aan dat een relatief tekort van n-3 LCP's inname door de moeder een rol kan spelen in de ontwikkeling van atopisch eczeem. Door de moeder wordt de n-3 
status van het kind bepaald, via aanvoer in de baarmoeder en in de moedermelk na de geboorte.

In hoofdstuk 3 wordt de opzet en de resultaten van een gerandomiseerde gecontroleerde trial (suppletie-studie) beschreven. De verwachting werd getest of toevoeging van borage olie welke rijk is aan GLA, aan de flesvoeding gedurende de eerste 6 levensmaanden, in een dosering vergelijkbaar met de inname die zuigelingen hebben via de borstvoeding. kan beschermen tegen de ontwikkeling van atopisch eczeem. De verwachting kwam voort uit de D6D-hypothese, omdat GLA het eerste omzettingsproduct is van linolzuur in de n-6 keten van essentiēle vetzuren, en toevoeging van buitenaf de veronderstelde gestoorde D6D omzetting zou omzeilen. Deelnemers aan dit onderzoek waren 118 flesgevoede kinderen van moeders met een voorgeschiedenis van atopische aandoeningen. De kinderen werden willekeurig toegewezen aan het GLA supplement of aan een placebo supplement. De intention-to-treat analyse toonde aan dat het risico op atopisch eczeem en de ernst van het eczeem lager leek te zijn in de GLA groep in vergelijking tot de placebogroep. Ook andere. secundaire atopische uitkomsten (jeuk en het gebruik van hormoonzalven) wezen in die richting. Echter, het effect was kleiner dan vooraf voorspeld en was niet statistisch significant (wat betekent dat de resultaten ook op toeval kunnen berusten). De GLA spiegels in de plasma fosfolipiden verdubbelde in de GLA groep in vergelijking met de placebo groep. We gebruikten deze toename van GLA in bloed als een maat voor therapietrouw en onderzochten of deze toename verband hield met de atopische uitkomsten. Deze verklarende analyse toonde aan dat een sterke stijging van GLA spiegels tussen één week en drie maanden geassocieerd was met een statistisch significant lagere emst van het eczeem op de leeftijd van één jaar. Omdat deze resultaten niet gebaseerd zijn op een intention-to-treat analyse is een oorzakelijk verband niet met zekerheid aangetoond en kan vertekening niet uitgesloten worden. De resultaten zijn systematisch nagekeken op vormen van vertekening, maar geen kon worden aangetoond. De resultaten zouden nog verklaard kunnen worden door een verschil in essentieel vetzuurmetabolisme tussen kinderen met en zonder atopisch eczeem, welke alleen tot uitdrukking komt na toevoeging van GLA aan de voeding. We achten dit echter niet waarschijnlijk omdat het in geen van de hypotheses past. We concluderen dat het geven van GLA aan kinderen met een verhoogd risico op atopisch eczeem de ernst van de symptomen vermindert, maar we kunnen dit niet staven met causaal bewijs.

We vonden in het observationeel onderzoek en de suppletiestudie opmerkelijke overeenkomsten met betrekking tot de uitkomstmaten. De resultaten van zowel het observationeel onderzoek als de suppletiestudie (hoofdstukken 2 en 3) geven aan dat essentiële vetzuren gerelateerd zijn aan de ernst van atopisch eczeem, maar er werd geen enkele relatie gevonden met totaal of specifiek $\mathrm{IgE}$. Daarom kunnen we concluderen dat essentiēle vetzuren geen directe rol spelen in de IgE aangestuurde deel van atopie bij de preventie van atopisch eczeem, maar wel een invloed hebben op de ernst van atopie in het eerste levensjaar. Dit is mogelijk gebaseerd op een ontstekingsremmend effect van essentiēle vetzuren zoals GLA en n-3 vetzuren. Dit wordt bevestigd door eerder onderzoek, dat heeft aangetoond dat toevoeging van GLA aan de voeding de spiegels van ontstekingsremmende metabolieten in de huid kan verhogen. 
Naast de $n-6 / n-3$ balans hypothese die de stijgende trend van atopische aandoeningen verklaarde, zijn in de laatste jaren meer hypotheses naar voren gekomen, die samengevat kunnen worden als de hygiêne-hypothese. Onderzoeken hebben aangetoond dat hooikoorts en atopisch eczeem minder vaak voorkomt bij kinderen die al oudere broers en/of zussen hebben. Dit effect wordt verklaard door de grotere kans op infecties door de aanwezigheid van oudere broers en zussen in grote families, welke een beschermende rol zouden spelen in de preventie van atopie; het zogenoemde 'sibling-effect'. Een recent onderzoek door Karmaus heeft echter aangetoond dat het effect van een hogere rang in geboorte al aantoonbaar is op IgE-spiegels in het navelstrengbloed. Omdat eerder is gebleken dat IgEspiegels in navelstrengbloed ook voorspellend zijn voor de ontwikkeling van latere atopie, zou het 'sibling-effect' zijn oorsprong dus al (gedeeltelijk) in de baarmoeder kunnen hebben. Als deze 'intra-uteriene sibling effect'-hypothese juist is zou neonataal IgE lager zijn naar mate de pariteit van de moeder (het aantal geborenen) hoger is. We hebben het onderzoek van Karmaus proberen te herhalen (hoofdstuk 4) en konden bevestigen dat een hogere rang in geboorte samengaat met lagere IgE spiegels op de leeftijd van één week. Nu kwam ook de vraag naar voren of de intra-uteriene essentiële vetzuurspiegels hierbij een rol spelen. Immers, ook de essentiële vetzuurspiegels van de moeder tijdens de zwangerschap worden bepaald door het aantal zwangerschappen dat zij eerder heeft gehad. We konden inderdaad aantonen dat pariteit geassocieerd was met een lagere DHA status van de moeder, maar niet van het kind.

Uitgaande van de huidige hypothese betreffende de $n-6 / n-3$ essentiële vetzuren zouden hogere gehaltes van $n-3$ vetzuren moeten beschermen tegen hoge $\lg E$ spiegels bij het kind. Op neonataal IgE konden wij zo'n effect niet aantonen. Wel vonden we seizoensinvloeden die een intra-uterien effect suggereren. Het lijkt erop dat de blootstelling aan pollen bij atopische zwangere vrouwen leidt tot intra-uteriene stimulatie van foetale IgE productie.

Onderzoeken waarin orale essentiële vetzuur-supplementen worden gegeven om atopisch eczeem te verlichten worden al tientallen jaren gedaan. Meestal worden supplementen gegeven met hoge doseringen van GLA, gewonnen uit teunisbloemolie of borage olie, of $n-3$ LCP's, gewonnen uit visolie. We hebben een meta-analyse uitgevoerd van deze onderzoeken (hoofdstuk 5) om het effect van de essentiële vetzuur-supplementen op de ernst van atopisch eczeem te schatten. De gebundelde effectmaat (effect size) voor GLA en $\mathrm{n}$-3 LCP's gaf geen duidelijk bewijs voor de werkzaamheid van GLA bij mensen met atopisch eczeem. De meeste onderzoeken waren van slechte kwaliteit, hadden weinig deelnemers, en/of beschreven de resultaten onvoldoende. Hierdoor moesten we conservatieve aannames doen bij de berekening van de gebundelde effectmaat. Dientengevolge kunnen we niet uitsluiten dat een klein effect van GLA suppletie op de emst van atopisch eczeem aanwezig is. Visolie had geen enkel positief effect op de ernst van atopisch eczeem.

In hoofdstuk 6 bespreken we de methodologie en de resultaten van het EFAtop onderzoek en de meta-analyse. We hebben aangetoond dat de klinische uitkomstmaten (de diagnose van atopisch eczeem en de ernst hiervan) niet correleerden met totaal serum IgE. Dit geeft aan dat er nog andere componenten betrokken zijn in de pathogenese van atopisch eczeem. De resultaten van zowel het observationeel onderzoek als de suppletiestudie suggereren dat essentiële vetzuren geen directe invloed hebben op de $\mathrm{lgE}$ gemedieerde component van atopie, maar mogelijk wel op de ontstekingscomponent zoals het tot uitdrukking komt in de 
ernst van atopisch eczeem in het eerste levensjaar mogelijk door een ontstekingsremmend effect. De meta-analyse gaf geen duidelijk bewijs voor de werkzaamheid van GLA bij mensen met atopisch eczeem, maar de onderzoeken die in de meta-analyse waren opgenomen waren voomamelijk uitgevoerd bij volwassenen met reeds bestaand eczeem. Derhalve is een effect bij jonge kinderen niet uitgesloten en dit past bij onze bevindingen dat essentiēle vetzuren vooral in de eerste maanden van belang zijn in de ontwikkeling van atopie.

Preventie van atopisch eczeem wordt het best bereikt door langdurig borstvoeding te geven. Voor sommige vrouwen is dit geen optie. Daarom zouden flesvoedingen verrijkt kunnen worden met $n-6$ en/of $n-3$ essentiêle vetzuren. Uit ons onderzoek komt echter niet overtuigend naar voren dat toevoeging van GLA aan flesvoeding effectief is. In het licht van de $n-6 / n-3$ hypothese is nog te weinig bekend over de optimale verhoudingen tussen de essentiële vetzuren.

Uiteindelijk worden de punten voor toekomstig onderzoek besproken. Momenteel worden er veel onderzoeken gedaan waarbij gekeken wordt naar de neurologische effecten van n-3 vetzuurtoevoeging aan flesvoeding. Om te kijken of essentielle vetzuren een positief effect hebben op atopisch eczeem in de vroege jeugd, kunnen de al lopende onderzoeken waarbij $n-3$ vetzuren aan de flesvoeding worden toegevoegd atopisch eczeem als secundaire uitkomstmaat meenemen. Een langere opvolging van observationele onderzoeken en gerandomiseerde gecontroleerde trials moet worden uitgevoerd om te bepalen of de resultaten die veelal worden gevonden in het eerste levensjaar ook aanwezig zijn als de kinderen ouder worden. 


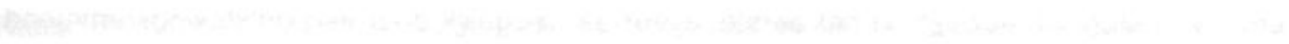

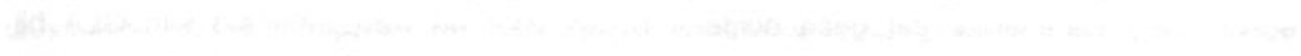

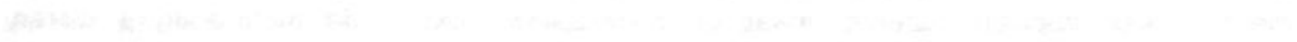

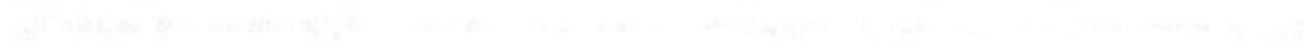

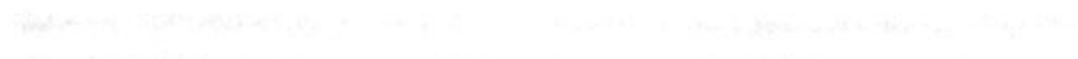

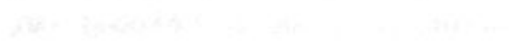

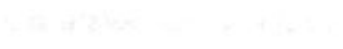

(1)

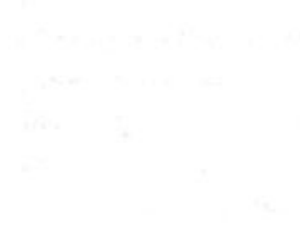

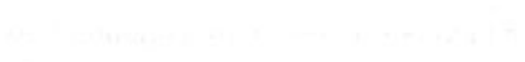

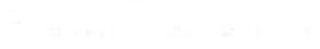

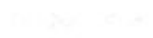
$\left(\frac{10}{2}\right.$

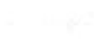
$\left(\frac{10}{2}\right.$ (1)

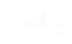
10

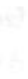

(

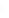




\section{DANKWOORD}

Op een woensdagavond, zomer 1992, op een terras op de Sint Pieterstraat te Maastricht bij de Waalse kerk, werd gesteld dat "The Essential Fatty Acids" een prima naam was voor een leuk bandje. Inmiddels zijn we 10 jaar verder en prijken deze woorden in de titel van dit boekje. Een band met deze naam bestaat zover bekend nog niet.

Dit boekje had hier niet voor $u$ gelegen als niet anderen met mij daaraan hadden meegewerkt. De traditie van een uitgebreid dankwoord in een proefschrift geeft de kans om deze mensen met name te noemen.

Allereerst wil ik al die verloskundigen welke voor de EVA-studie hebben geworven bedanken. Zonder jullie inzet waren we nooit aan alle ouders en kinderen gekomen die mee wilden doen.

Dit brengt me vervolgens bij alle ouders die hun toestemming hebben gegeven om mee te doen met hun kinderen. Ik heb het plezier gehad om bij velen van jullie thuis te mogen langskomen. Ik spreek ook namens Alice en Manon als ik jullie bedank voor de gastvrijheid en het vertrouwen dat jullie in ons hadden. Ik ben me er van bewust dat het niet altijd gemakkelijk geweest is om je gezonde kind te laten prikken. Daarbij wil ik zeker niet de helft van de ouders en hun kinderen vergeten die om wat voor reden dan ook niet meer verder konden meedoen aan het onderzoek.

Carel, ik wil je danken voor het vertrouwen dat je in me stelde. Onze samenwerking en inspanningen hebben uiteindelijk geleid tot dit mooie boekwerkje.

Piet, je hebt met kritisch oog op de achtergrond de grote lijnen gevolgd en je commentaar zette de puntjes op de i. Pieter, bedankt voor het corrigeren van de tekst en je zinvol commentaar op alle stukken die ik bij je mocht inleveren.

Alice, Manon, Annemie, Diane en Hilde. Een speciaal dankwoord voor jullie is zeker op zijn plaats: al die tijd van bellen, prikken, werven, vragenlijsten afnemen, administreren, vergaderen, kopiëren en bezoeken heeft zijn vruchten afgeworpen. Een proefschrift schrijven is veelal ook een kwestie van teamwork. Het was een waar genoegen om met jullie het EVAteam te vormen en met jullie samen te mogen hebben gewerkt. Wim, bedankt voor de logo's die je hebt verzorgd. Fons, je hebt voor ons als interne monitor de zaken uitstekend waargenomen.

Rian bedankt voor je betrokkenheid bij het project. De eerste stappen op het essentieel vetzuur-pad heb ik met jou gezet. Zoals je merkte heeft je enthousiasme aanstekelijk gewerkt. Hasibe, bedankt voor het uitwerken van al die vetzuurmonsters en de gezelligheid op het lab.

Beste Charles en Jaap. Jullie klinische kennis en jullie bereidheid om de SCORAD uit te proberen in de beginfase heeft voor mij een goed inzicht gegeven in datgene waar ik mee bezig was. Charles, ik heb met enorm plezier met je samengewerkt in de hectiek van de kinderpoli's. Ik heb dit stuk 'veldwerk' zeer gewaardeerd. 
Alle medewerkers van de kinderpoli van het azM wil ik bedanken voor de gastvrijheid die we twee-en-een-half jaar lang hebben mogen genieten op de dinsdagmiddagen. Drie medewerkers en een aantal extra bezoekers erbij moet voor jullie heel wat drukte hebben opgeleverd. We hebben ons altijd welkom gevoeld.

Steven en Janny; een paar honderdtal vloeitjes met bloedspots zijn jullie kant op gegaan. De communicatie was altijd direct en duidelijk. Dit is een zeer prettige manier van samenwerken geweest en heeft een onuitwisbare indruk achtergelaten (met name in kamer 0.025).

Beste Paul en Nancy, de samenwerking met een lab in het ziekenhuis leek mij in het begin heel ingewikkeld en omslachtig. Jullie hebben voor me het tegendeel bewezen. Dank voor jullie inzet, het persoonlijke contact en het uitwerken van de monsters.

José, Brigitte en Mary, jullie professionaliteit in het prikken van kleine kinderen is zeer op prijs gesteld. Dank dat jullie die dinsdagmiddagen voor ons klaar stonden. Leuk dat we nog even samen mochten werken op het busje Brigitte!

Désiree, je was van het begin als verloskundige bij het project betrokken. Je realistische kijk op zaken heeft veel steun gegeven. Ik ben blij dat je bij de voltooiing van dat andere project, de geboorte van onze Huub, aanwezig kon zijn!

Beste Audrey, je kwam in de eindfase op het project met je eigen deelonderzoek. Je hebt jezelf overtuigend bewezen. Succes met je komende loopbaan!

Cobie, in goed overleg hebben we de lay-out op een rij gekregen. Ik heb met een gerust hart alles in je handen kunnen laten in de laatste fase. Anja, bedankt voor het regelen en afhandelen van al die administratieve rompslomp toen ik me 'plots' met wat anders bezig moest houden.

Ik wil met name ook die mensen bedanken die buiten het project om hun betrokkenheid hebben getoond: Kaat en Mirian (dank voor jullie nuchtere kijk op zaken), Ischa en Emilie (ook een traditie kent een eerste keer), Danielle, Nicole, Ute, (de PVA-stoel heeft z'n nut bewezen), Hannerieke (voor de ditjes en datjes die net zo belangrijk zijn), Foekje, Rob, Luc, Carolien, Conny, Jolanda, Jos, Gerard, Kim, Matty, Maurice en Ria.

Medewerkers van KDV de Flex en OBS Idefix; werken was niet mogelijk geweest zonder dat ik met een gerust hart mijn kinderen in jullie goede, flexibele, zorg kon achterlaten. Bedankt!

Echte vrienden kom je zelden tegen, Rose, Monique, Gon, de Mariëlles en Soni. Al 20 jaar kan ik op jullie vriendschap terugvallen. De doc-hok reünie de komende jaren ben ik weer van de partij. Rose, bedankt voor de ladies-night-outings, je goede keus voor films met humor en je nooit aflatende oor. Op een of andere manier belde je me altijd net wanneer ik een goed gesprek met een niet-wetenschapper nodig had. Monique, ook jij bedankt voor die eindeloze avonden aan de telefoon en je steun als ex-AIO. Nu je dichterbij woont en dit boekje z'n uiteindelijke vorm heeft gekregen kom ik weer eens vaker lijfelijk langs. 
Piep! We hadden samen ons feestje kunnen vieren... Het is wel geen barbecue op Fort St. P. geworden... Jouw nuchtere, humoristische en juridische kijk op zaken heeft goed geholpen. Dat onze vriendschap nog lang mag blijven duren.

$\mathrm{Pa}$, ons ma en jij hebben me de basis mee kunnen geven voor het boekje dat hier ligt. Dank je wel dat wat ik ook deed en waar ik ook naar toe ging, al was het naar de andere kant van de wereld, jullie altijd voor me klaar stonden.

Marcel, zonder jouw onvoorwaardelijke steun en inzet thuis had dit nooit gekund. Bedaank, leef!

Lieve Myrke, lieve Sophie, lieve Huub, de keuzes voor jullie zijn de beste ooit in mijn leven geweest. Niets is zo leuk als jullie op je pad te begeleiden en die oneindige stroom vragen over het hoe en waarom en waartoe, en de juiste antwoorden daarop te vinden.

Deze woensdagavond, herfst 2002, feest! 


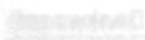

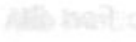

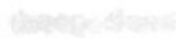

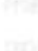

xan

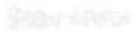




\section{CURRICULUM VITAE}

Christel van Gool werd geboren in Dongen in $1968 . \mathrm{Na}$ in 1988 aan het St. Oelbertgymnasium in Oosterhout (NB) haar diploma te hebben gehaald, begon ze aan de studie Gezondheidswetenschappen aan de Universiteit Maastricht. In 1995 studeerde ze af, met als afstudeerrichtingen Bewegingswetenschappen en Biologische Gezondheidkunde. Tijdens haar studie verrichtte ze onderzoek bij de capaciteitsgroep Humane Biologie waar ze onderzoek deed naar de essentiēle vetzuurstatus van kinderen met phenylketonurie. Daarnaast heeft ze, voor haar studie Bewegingswetenschappen, onderzoek verricht in Melbourne, Australiē, op de Respiratory Function Unit van het Heidelberg Repatriation Hospital. Hier deed ze onderzoek naar de rol van lactaat bij het trainen van patiènten met ernstig COPD. In 1996 kreeg ze een functie op de capaciteitsgroep Epidemiologie van de Universiteit Maastricht als onderzoeksassistent. Tussen maart 1997 en juli 2002 werkte zij als assistent in opleiding aan de volbrenging van dit proefschrift bij desbetreffende capaciteitsgroep. Momenteel is ze werkzaam als onderzoeker in het academisch ziekenhuis Maastricht.

\section{ABOUT THE AUTHOR}

Christel van Gool was born in Dongen in 1968. After completing her secondary education at the St Oelbertgymnasium in Oosterhout (province of Noord Brabant) in 1988, she studied Health Sciences at the University Maastricht. In 1995 she graduated, with Human Movement Sciences and Biological Health Sciences as her specialisations. During her studies she did research at the department of Human Biology where she studied the essential fatty acid status of phenylketonuric children. She also studied role of lactate in training severe COPD patients at the Respiratory Function Unit of the Heidelberg Repatriation Hospital in Melbourne, Australia, under supervision of the department of Human Movement Sciences, University Maastricht. In 1996 she started working as a research assistant at the department of Epidemiology of Maastricht University. Between March 1997 and July 2002 she worked at this department on this thesis. At this moment she works at the University Hospital Maastricht as a researcher. 
Atopic diseases such as atopic eczema are increasing worldwide. The perinatal period is thought to be a critical period in the development of atopy, and previous studies have suggested that essential fatty acids may be involved in the etiology.

This thesis presents the results of the EFAtop study that focused on the role of essential fatty acids on early development of atopy. To this end we have performed two major studies: an observational study and a randomized controlled trial. The observational study was performed in a group of breast-fed infants, in which we studied the telation between perinatal essential fatty acid status in mother and infant and the development of atopic eczema at the age of one year. In the randomized controlled trial either gamma-linolenic acid or a placebo was supplemented to infants from one week to six months to evaluate the role of dietary n-6 EFAs. Furthermore we present a meta-analysis of essential fatty acids on existing atopic dermatitis. 